\title{
An Xist-dependent protein assembly mediates Xist localization and gene silencing
}

Amy Pandya-Jones ${ }^{1}$, Yolanda Markaki ${ }^{1,4}$, Jacques Serizay ${ }^{1 \#,}$, Tsotne Chitiashvilli1,5, Walter

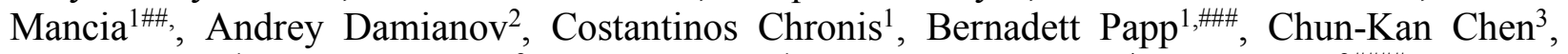
Robin McKee ${ }^{1}$, Xiao-Jun Wang ${ }^{2}$, Anthony Chau ${ }^{1}$, Heinrich Leonhardt ${ }^{4}$, Sika Zheng ${ }^{2 \# \# \#}$, Mitchell Guttman $^{3}$, Douglas. L. Black ${ }^{2,5^{*}}$ and Kathrin Plath ${ }^{1,5^{*}}$

1. Department of Biological Chemistry, University of California, Los Angeles, Los Angeles, CA 90095, USA

2. Department of Microbiology, Immunology and Molecular Genetics, University of California, Los Angeles, Los Angeles, CA 90095, USA

3. Division of Biology and Biological Engineering, California Institute of Technology, Pasadena, CA 91125, USA

4. Department of Biology and Center for Integrated Protein Science, LMU Munich, 82152 Munich, Germany

5. Molecular Biology Institute, Jonsson Comprehensive Cancer Center, Brain Research Institute, Graduate Program in the Biosciences, Eli and Edythe Broad Center of Regenerative Medicine and Stem Cell Research, David Geffen School of Medicine at the University of California Los Angeles, Los Angeles, CA 90095, USA

* Correspondence to: kplath@mednet.ucla.edu and dougb@microbio.ucla.edu

\# École Normale Supérieure de Cachan, Université Paris-Saclay, Saclay, France. Current Address: The Gurdon Institute and Department of Genetics University of Cambridge, Cambridge CB2 1QN, United Kingdom

\#\# Current Address: Department of Neurological Surgery, University of California San Francisco, San Francisco, CA 94143, USA

\#\#\# Current Address: Department of Oral Biology, College of Dentistry, University of Florida, Gainesville FL 32610, USA.

\#\#\# Current Address: Division of Biomedical Science, University of California, Riverside, Riverside, CA 92521, USA 


\section{Summary}

Nuclear compartments play diverse roles in regulating gene expression, yet the molecular forces and components driving compartment formation are not well understood. Studying how the lncRNA Xist establishes the inactive-X-chromosome (Xi)-compartment, we found that the Xist RNA-binding-proteins PTBP1, MATR3, TDP43, and CELF1 form a condensate to create an Xidomain that can be sustained in the absence of Xist. The E-repeat-sequence of Xist serves a multivalent binding-platform for these proteins. Without the E-repeat, Xist initially coats the Xchromosome during XCI onset but subsequently disperses across the nucleus with loss of gene silencing. Recruitment of PTBP1, MATR3, TDP-43 or CELF1 to $\triangle \mathrm{E}-X i s t$ rescues these phenotypes, and requires both self-association of MATR3 and TDP-43 and a heterotypic PTBP1MATR3-interaction. Together, our data reveal that Xist sequesters itself within the Xi-territory and perpetuates gene silencing by seeding a protein-condensate. Our findings uncover an unanticipated mechanism for epigenetic memory and elucidate the interplay between RNA and RNA-bindingproteins in creating compartments for gene regulation.

\section{Main text}

The function of long non-coding RNAs (lncRNAs) and the mechanisms by which they act remain largely unknown. One of the best studied lncRNAs is Xist, which orchestrates X-chromosome inactivation (XCI) in placental female mammals ${ }^{1-7}$. By spreading across one X-chromosome and mediating chromosome-wide gene silencing, Xist equalizes X-linked gene expression with that of males $^{8-12}$. XCI initiates when Xist is induced on one of the two X-chromosomes in pluripotent cells of the implanting blastocyst, or upon induction of differentiation in embryonic stem cells $(\mathrm{ESCs})^{4,13}$, the latter providing a powerful model for the mechanistic dissection of XCI-initiation. Intriguingly, Xist shapes nuclear organization during XCI-initiation. Xist establishes a transcriptionally silent, intra-chromosomal domain (or compartment) by specifically localizing to the X-chromosome from which it is transcribed and inducing the compaction of the forming inactive $\mathrm{X}$-chromosome $(\mathrm{Xi})$, the enrichment of heterochromatin proteins, the repositioning of silenced genes into the center of the $\mathrm{Xi}$, and the exclusion of active transcriptional regulators, such as RNA polymerase $\mathrm{II}^{1,2,14-20}$. Yet, the mechanisms that drive and maintain the Xist RNA within a spatially confined region to establish this $\mathrm{Xi}$-domain remain unclear. 
Mechanistically, Xist is thought to act as a modular scaffold for diverse proteins each confering discrete functions on the RNA. These functions include chromatin localization, gene silencing, recruitment of chromatin modifiers, and sub-nuclear-positioning of the $\mathrm{Xi}^{6,14,20-27}$. Consistent with this model, functional regions within the RNA, structured as six conserved repeat arrays termed $\mathrm{A}-\mathrm{F}$, have been identified ${ }^{1,6,28-33}$, along with many interacting proteins $\mathrm{s}^{22,25,34}$ and critical RNA-protein interactions ${ }^{21,22,25,29,35,36}$. However, which of the Xist-interacting proteins play roles in formation of the Xi-domain and the spatial confinement of Xist is unclear. Interestingly, during the initiation of XCI, but after initial spreading and gene silencing (within $72 \mathrm{~h}$ of ESC differentiation), a state is reached where gene silencing remains stable even if Xist is experimentally turned off ${ }^{5}$. This transition during XCI initiation from Xist-dependent to Xistindependent gene silencing ${ }^{5}$ cannot currently be explained. This is partially due to the fact that $X i s t$-interacting proteins and functional Xist domains have primarily been examined during the Xist-dependent phase of XCI initiation.

Among the Xist-interacting proteins are RNA-binding proteins (RBPs) with known functions in regulating mRNA processing, including the splicing regulators Polypyrimidine Tract Binding Protein 1 (PTBP1), MATRIN-3 (MATR3), CUG-Binding Protein 1 (CELF1), and TARDNA Binding Protein (TDP-43) ${ }^{22,25,34}$. X-linked gene silencing is largely unaffected by siRNAmediated depletion of these factors during the Xist-dependent phase of XCI initiation (Extended Data. Fig. 1a-c) ${ }^{25,37}$, raising the question of what roles these proteins play in XCI. Interestingly, these RBPs have all been shown to form extensive higher-order assemblies, particularly when concentrated by RNAs containing multivalent protein binding sites ${ }^{38-43,43}$. Because Xist contains several highly repetitive sequences that could seed a high spatial concentration of such proteins, we hypothesized that interactions between Xist and proteins like PTBP1, MATR3, CELF1, and TDP-43 might create a higher-order assembly within the Xi to establish a nuclear compartment that functions to restrict Xist localization and enforce the silent state of the $\mathrm{Xi}$.

Based upon our hypothesis, we first examined whether the depletion of PTBP1, MATR3, CELF1, or TDP-43 has an impact on Xist localization. To test this, we knocked down PTBP1, MATR3, CELF1, or TDP-43 at day 3 of female ESC differentiation (during the Xist-dependent phase of XCI), and observed nuclear dispersal of Xist compared to a control siRNA (Fig. 1a/b). To 
quantify the Xist dispersal, we derived the Xist aggregation score, by normalizing the projected Xist RNA fluorescent in situ hybridization (FISH) signal by the smallest circular area encompassing the Xist FISH mask (Fig. 1c and Extended Data Fig. 1d/e). Xist dispersal occurred with small or no changes in Xist transcript levels and splicing (Extended Data Fig. 1f-i). Consistent with this finding, PTBP1 knockdown in male ESCs expressing Xist from a cDNA transgene, which does not require a splicing event, resulted in a similar dispersal of the Xist RNA FISH signal (Extended Data Fig. 2). Depletion of PTBP1, MATR3, CELF1 or TDP-43 also revealed defects in the enrichment of heterochromatin marks on the forming Xi (Extended Data Fig. 1j/k). These findings demonstrate that PTBP1, MATR3, CELF1, and TDP-43 play a role in the enrichment of Xist within the X-chromosome territory, which is largely independent of their RNA-processing activities.

As these findings indicated a role for these RBPs in controlling Xist localization, we next determined where on Xist these factors bind, by applying CrossLinking and ImmunoPrecipitation of RNA followed by high-throughput sequencing (CLIP-Seq). CLIP-seq for PTBP1, MATR3 and CELF1 was carried out in male ESCs that can inducibly express endogenous Xist, a model system for studying the Xist-dependent phase of XCI initiation ${ }^{24}$. Each of the three RBPs yielded a striking enrichment of reads on the E-repeat of Xist (Fig. 1d(i) and Extended Data Fig. 3a). Consistent with the binding signature of these proteins, the $1.4 \mathrm{~kb}$ E-repeat comprises over $50 \mathrm{C} / \mathrm{U} / \mathrm{G}$-rich elements predicted to serve as PTBP1, MATR3 and CELF binding sites (Extended Data Fig. 3b-d) ${ }^{44-46}$. The binding of PTBP1 molecules to the E-repeat RNA was further confirmed with recombinant PTBP1 and in vitro transcribed E-repeat RNA in electrophoretic mobility shift assays (Fig. 1e). The Erepeat thus serves as a multivalent binding platform for PTBP1, MATR3, and CELF1. As Xist was expressed for just 6 hours prior to fixing cells for CLIP, our results indicated that these factors associate with Xist at the earliest stage of XCI initiation. To determine whether this interaction occurs co-transcriptionally, we performed Chromatin Immunoprecipitation followed by highthroughput sequencing (ChIP-seq) against PTBP1 and observed binding primarily over the genomic region encoding the E-repeat, upon induction of Xist expression, but not before (Fig. 1d(ii)). Taken together, these results demonstrate that PTBP1, and likely MATR3 and CELF1, engage Xist as it is transcribed. 
To ascertain whether these factors also associate with Xist in cells with an established Xi, we examined published datasets. The PTBP1 CLIP-seq profile for Xist in differentiated female cells is strikingly similar to that observed for PTBP1 in ESCs, as is the CLIP-seq signature against PTBP2, the neural homolog of PTBP $1^{47}$ (Fig. 1d(iii)). We further found that TDP-43 CLIP-seq in embryonic mouse brain displayed binding at the $3^{\prime}$ end of the E-repeat, where multiple (GU)n tracts presumably serve as TDP-43 binding motifs (Fig. 1d(iii) and Extended Data Fig. 3b/c) ${ }^{48,49}$. These results indicate that binding of TDP-43 and PTBP1 to the E-repeat persists after XCI initiation is completed and that PTBP2 likely replaces PTBP1 on Xist RNA in brain ${ }^{47}$. We conclude that Xist is a multivalent binding platform for PTBP1, MATR3, CELF1, TDP-43 and their homologs throughout all stages of XCI.

Next, we applied immunofluorescent staining to differentiating female ESCs to determine whether recruitment of PTBP1, MATR3, CELF1 or TDP-43 by Xist could be microscopically oberved within the $\mathrm{Xi}$. These experiments revealed that CELF1 accumulates on the $\mathrm{Xi}$, which was delineated by the enrichment of $\mathrm{H} 3 \mathrm{~K} 27 \mathrm{me} 3$, and that its accumulation increases in intensity from day 3 to day 7 of differentiation (Fig. 1f-i). We also observed PTBP1 to concentrate within the Xiterritory at later times of differentiation (day 7), in a mesh-like pattern distinct from the punctate staining seen earlier (day 3) (Extended Data Fig. 4a). Although clearly present in the Xi, MATR3 and TDP-43 did not appear enriched in the Xi relative to the nuclear background (Extended Data Fig. 4b/c). Xi-enrichment of these RBPs may not have been detected due to inassessibility of their epitopes or because high concentrations are present elsewhere in the nucleus. These findings indicate that, CELF1 and PTBP1 concentrate over time within the Xi-territory, consistent with the time-dependent formation of a spatially concentrated assembly.

We reasoned that, if PTBP1, MATR3, CELF1 or TDP-43 control the Xi-accumulation of Xist, loss of the E-repeat should disrupt XCI by reducing Xist enrichment within the Xchromosome territory. In support of this hypothesis, it has been shown that Xist lacking Xist exon 7, which contains the E-repeat (Fig. 2a), displays compromised nuclear localization during XCI initiation $^{32,33}$. To directly test the requirement of the E-repeat in Xist localization, we deleted it in a female hybrid mouse ESC line that carries one X-chromosome from the 129 background and a second X-chromosome from the castaneous (cas) background. We confirmed that the deletion of 
the E-repeat occurred on the 129 allele, which also harbors 11 copies of an MS2-RNA tag within $X i s t^{15}$, yielding the $\mathrm{X}_{129}{ }^{\mathrm{Xist}} \Delta \mathrm{E}, \mathrm{MS} 2 \mathrm{X}_{\text {Cas }} \mathrm{Xist}^{\mathrm{WT}}$ genotype (referred to as $\Delta \mathrm{E}$ ESCs below) (Fig. 2a and Extended Data Fig. 5a-d). We ensured that $\triangle \mathrm{E}$ ESCs maintained two X-chromosomes, and differentiated normally, as judged by morphological changes and loss of NANOG expression (Extended Data Fig. 5e-g). When transcribed from the $\mathrm{X}_{129}{ }^{\mathrm{Xist}} \Delta \mathrm{E}, \mathrm{MS} 2$ allele, Xist exon 6 was spliced to a cryptic site within exon 7 to generate an RNA missing specifically the E-repeat (Extended Data Fig. 6).

RNA FISH over seven days of differentiation (Fig. 2b) revealed that the number of cells containing an Xist-coated X-chromosome increased gradually until day 4 of differentiation in both WT and $\Delta$ E cells (Fig. 2c). At later time points (days 5-7), we observed a significant decline in the proportion of cells containing an Xist enrichment in $\Delta \mathrm{E}$ compared to WT cells $(>50 \%$ reduction at day 7) (Fig. 2c). This reduction was specific to the $129^{\mathrm{Xist}} \Delta \mathrm{E}, \mathrm{MS} 2$ allele as revealed by RNA FISH against the MS2 tag (Fig. 2d). Although slightly less than in WT cells, the level of the MS2+Xist transcripts in $\Delta \mathrm{E}$ cells continually increased during differentiation and RNA half-life was the same for WT and $\Delta \mathrm{E}$ Xist measured at day 3 of differentiation (Extended Data Fig. 7). Therefore, loss of $\Delta \mathrm{E}$ Xist enrichment on the $\mathrm{Xi}$ was not an apparent consequence of decreased Xist transcript abundance or reduced RNA stability.

Early during initiation of XCI (differentiation day 3), the $\Delta \mathrm{E}$ Xist was observed to enrich over the X-chromosome, while its aggregation measurements revealed a modest, but significant, defect in $\Delta \mathrm{E}$ Xist localization compared to WT Xist (Fig. 2e/f). RNA antisense purification (RAP) of Xist and sequencing of the associated DNA in male ESCs inducibly expressing full length or $\Delta \mathrm{E}$ Xist revealed highly correlated patterns of interaction across the X-chromosome early after Xist induction (6h induction, Pearson, $\mathrm{r}=0.85$, Fig. $2 \mathrm{~g}$ ). This confirms that the E-repeat is not required for the initial transfer of Xist across the X-chromosome upon onset of XCI. By day 7, however, $\Delta \mathrm{E}$ Xist was strikingly dispersed within the nucleus, often localizing at the nuclear lamina (Fig. 2h and Extended Data Fig. 8a-d). The $\Delta \mathrm{E}$ Xist aggregation score decreased to less than half of WT by day 7 (p<0.00001, 2-sample ks test) (Fig. 2f and Extended Data Fig. 8e/f). H3K27me3 enrichment on the $\mathrm{Xi}$ is an Xist-dependent mark at all stages of $\mathrm{XCI}^{17,26}$. Consistent with the Xist localization 
defect later in XCI initiation, we observed lower H3K27me3 enrichment and reduced chromatin compaction over the X-chromosome territory at day 7 of differentiation, despite having been established normally at day 3 of differentiation (Extended Data Fig. 9/10). Notably, the $\Delta \mathrm{E}-X i s t$ localization phenotype that becomes starkly apparent between day 4 and 7 of differentiation follows the transition from the Xist-dependent to the Xist-independent phase of XCI initiation ${ }^{5}$, and coincides with the described decrease in Xi-enrichment of the H3K27me3 methyltransferase complex PRC2 ${ }^{17,50}$ (Extended Data Fig. 11). Together, these results reveal a transition in the mechanisms that enrich Xist on the X-chromosome during XCI initiation, from a largely E-repeatindependent to an E-repeat-dependent phase. Moreover, the inability of Xist to remain associated with the X-chromosome in the absence of the E-repeat occurs after the induction of transcriptional silencing (see below), and upon transition to the Xist-independent-phase of XCI.

The Xist RNA signal has been previously resolved into 100-200 individual foci (or granules) when assessed with super-resolution imaging by 3D-SIM ${ }^{18}$. Quantifying the number of foci, we found that MS2+Xist ${ }^{129}$ clouds at both D3 and D7 in WT cells, as well as the MS2+Xist ${ }^{129}$ clouds in $\Delta \mathrm{E}$ cells at D3, were comprised of 200 foci (Fig. 2i). However, at D7, we observed a striking $50 \%$ increase in the average number of $\mathrm{MS} 2+X i s t^{129}$ foci in $\Delta \mathrm{E}$ compared to WT cells ( $\mathrm{p}=0.05$, two-sample ks-test) (Fig. 2i). This change occurs after the number of cells with Xist clouds in the population has stabilized (Fig. 2c) and while Xist transcript abundance is still increasing (Extended Data Fig. 7a). Thus, the increased number of $\Delta \mathrm{E} X i s t$ foci at later times may result from disassembly of Xist foci that each contain multiple Xist transcripts ${ }^{51,52}$. Taken together, our imaging data support a model in which the E-repeat is required for integration of multiple Xist transcripts into individual Xist foci and for stabilizing the localization of these foci within the Xchromosome.

The delayed timing of defective $\Delta \mathrm{E}$ Xist localization raised several possibilities regarding gene regulation. Silencing of genes on the $\Delta \mathrm{E}$ Xist-expressing X-chromosome (i) might not occur, (ii) might be initiated but not sustained, or (iii) might be both initiated and propagated normally with $\Delta \mathrm{E}$ Xist dispersal occuring after silencing becomes Xist-independent ${ }^{5}$. To distinguish between these possibilities, we examined differentiating WT and $\triangle \mathrm{E}$ ESCs using RNA FISH to measure nascent transcripts from five X-linked genes known to be transcriptionally silenced by XCI: Gpc4, 
Rnf12, Mecp2, Chic1, and Atrx (Fig. 3a/b). The $\Delta \mathrm{E}$ Xist RNA was identified by FISH against the MS2 tag. In this assay, the presence of two nuclear nascent transcript foci is indicative of biallelic expression of the respective X-linked gene. One focus indicates silencing of one allele, which can be correlated to the Xi (Xist-expressing allele) by colocalization. In WT cells by day 3 of differentiation, some genes (Gpc4 and Rnf12) already show mono-allelic and others (Atrx and Mecp2) biallelic expression, consistent with known differences in silencing kinetics (Fig. 3c and Extended Data Fig. 12a-d $)^{53}$. Importantly, during the first three days of differentiation, we observed little difference in the level of gene silencing between WT and $\Delta \mathrm{E}$ cells for most genes (Fig. 3c (compare top and bottom panels)/3d and Extended Data Fig. 12d ), although, silencing of Tsix, a repressor of Xist $t^{54}$, and Gpc4 were slightly delayed compared to WT (Fig. 3c and Extended Data Fig. 12c-e). Later stages of differentiation (day 4-7) presented a strikingly different pattern. The $\Delta \mathrm{E}$ Xist expressing cells frequently failed to silence the five X-linked genes (Fig. 3c/d and Extended Data Fig. 12c/d). Consistent with this late silencing defect, we observed that RNApolymerase II (RNA-PolII), which was largely excluded from the $\Delta \mathrm{E}$ Xist-marked territory during early differentiation, was intermingled with the $\Delta \mathrm{E}-X i s t$ foci at later times (Fig. 3e and Extended Data Fig. 11b-d). The E-repeat is thus essential for sustaining silencing of X-linked genes and exclusion of RNA-PolII beyond the initial wave of transcriptional shutoff. Moreover, the results demonstrate that the Xist-independent state of XCI initiation ${ }^{5}$ is not established in the absence of the E-repeat, suggesting that Xist generates the epigenetic memory for the silent state through the E-repeat. These findings uncover a new and crucial layer of regulation during Xist-dependent silencing.

To test a causal relationship between the E-repeat-binding RBPs, Xist localization and gene silencing, we synthetically fused PTBP1, MATR3, CELF1, or TDP-43 to the MS2-coat protein $(\mathrm{MCP})^{55}$. This allowed recruitment of these proteins to $\Delta \mathrm{E}$-Xist RNA during differentiation via the 11xMS2-tag (Fig. 2a, 4a and Extended Data Fig. 13a, c, d). In this gain-of-function approach, we were able to differentiate ESCs for seven days, which was not possible with RBP depletion. We then assessed whether synthetic recruitment of an RBP rescued the phenotypes associated with the loss of the E-repeat. We first confirmed in vivo recruitment of the synthetic protein to $129^{\mathrm{MS} 2}$ Xist by expressing a Flag-MCP-GFP fusion protein in differentiating WT ESCs (Extended Data Fig. 13b). We found that continued expression of a Flag-MCP-PTBP1 fusion protein in $\triangle \mathrm{E}$ cells, 
assessed at day 7 of differentiation, rescued Xist localization, gene silencing of Gpc4 and Atrx, and H3K27me3 enrichment on the $129^{\Delta \mathrm{E}, \mathrm{MS} 2} \mathrm{X}$-chromosome at day 7 of differentiation (Fig. 4b-e and Extended Data Fig. 13e). Interestingly, we observed a similar rescue upon expression of FlagMCP-MATR3, Flag-MCP-TDP-43, or Flag-MCP-CELF1 (Fig. 4b-e and Extended Data Fig. 13e). These data demonstrate that the E-repeat controls Xist localization, gene silencing, and heterochromatin formation via its binding proteins PTBP1, MATR3, TDP-43 and CELF1.

The ability of PTBP1, MATR3, TDP-43 and CELF1 to individually rescue phenotypes associated with loss of the E-repeat raised the question of whether they act redundantly in this process. To examine this more closely, we took advantage of a known direct interaction between PTBP1 and MATR3 ${ }^{44}$. We found that Flag-MCP-MATR3 harboring a mutant PTBP1-RRM Interaction (PRI) sequence (MATR3mPRI) ${ }^{44}$, partially rescued H3K27me3 enrichment, but was unable to rescue the Xist localization and gene silencing defects observed upon loss of the E-repeat (Fig. 4b,d,e and Extended Data Fig. 13e/f). The converse mutation in PTBP1 (Y247Q) that prevents interaction of PTBP1 with MATR $3^{44}$, was similarly unable to rescue the aggregation and silencing phenotypes associated with loss of the E-repeat (Fig. 4b-e and Extended Data Fig. 13e). These findings are supported by co-immunoprecipitation results demonstrating that PTBP1, MATR3, CELF1 and TDP-43 can interact with one another in the presence of RNA, whereas only PTBP1 and MATR3 robustly co-precipitate after RNase treatment (Fig. 4f and Extended Data Fig. 14). These findings show that PTBP1 and MATR3 are each necessary but insufficient for conferring function on the E-repeat and do not act completely redundantly in this process. Moreover, the data demonstrate that a specific protein-protein interaction between PTBP1 and MATR3 is critical for Xist localization, gene silencing and H3K27me3 Xi-enrichment.

The protein CIZ1 was previously suggested to anchor Xist to chromatin via the E-repeat in mouse embryonic fibroblasts (MEFs) ${ }^{31,56}$. Interestingly, PTBP1 and MATR3 interact with CIZ1 in both the presence and absence of RNA in our co-immunoprecipitation assay (Fig. 4f and Extended Data. Fig. 14). However, expression of Flag-MCP-CIZ1 did not rescue Xist cloud formation or X-linked gene silencing in our rescue system (Extended Data Fig. 15). Moreover, unlike the CIZ1 Xi-enrichment observed in WT cells (Extended Data Fig. 16) ${ }^{31,56}$, CIZ1 did not accumulate on the $\Delta \mathrm{E}$-Xist-coated X-chromosome in cells expressing Flag-MCP-PTBP1, - 
MATR3, or -TDP-43 (Extended Data Fig. 16). Despite the RNA-independent interactions observed between CIZ1 and PTBP1/MATR3, these results suggest that distinct functional complexes assemble on the E-repeat and that rescue by PTBP1, MATR3, TDP-43 and CELF1 is independent of CIZ1. As a negative control, we also tested a bivalent MS2-CP-GFP-MS2-CP fusion in our rescue system (Extended Data Fig. 17), which was unable to rescue the $\Delta \mathrm{E}-X i s t$ localization and silencing defects. This result suggests that the linkage formed PTBP1 and MATR3 does not function to simply tether Xist transcripts together and that additional activities conferred by the recruited proteins likely facilitate compartmentalization of Xist and downstream events in XCI.

To define such additional features of the E-repeat-interacting RBPs, we performed a limited domain analysis. MATR3 encodes two zinc finger domains described as conferring DNA binding ${ }^{57}$ (Fig $4 \mathrm{~b}$ ). We found that the rescue of the $\Delta \mathrm{E}$-Xist phenotypes by MATR3 is independent of these Zinc finger domains (MATR3 $\Delta$ Zfn) (Fig. 4b,d,e and Extended Data Fig. 13e/f). We also tested whether the valency of the RNA binding by PTBP1 affects the ability of the protein to confer function on the E-repeat. PTBP1 lacking two of its four RNA recognition motifs (RRMs) $(\Delta \mathrm{C}$ PTBP1 without RRM 3 and 4, Fig. 4b) retains some splicing repression activity ${ }^{58}$ and, in our system, rescued Xist cloud formation, H3K27me3 Xi-enrichment, and gene silencing defects observed upon E-repeat deletion (Fig. 4c-e and Extended Data Fig. 13e). However, closer inspection of $\triangle \mathrm{E}$ Xist clouds formed in the $\triangle \mathrm{C}$-PTBP1 rescue line, however, revealed the presence of dispersed Xist foci within the nucleus, normally not seen for WT Xist or in the rescue with fulllength PTBP1 (see inset, Fig. 4c). This result indicates that binding valency by PTBP1 is an important parameter in the function of the PTBP1-Xist assembly and suggests that PTBP1 likely uses all of its RRMs to build a network of RNA-protein interactions in the Xi (Extended Data Fig. 4).

The formation of the Xist territory containing PTBP1 is interesting in light of the observation that PTBP1 can undergo liquid-liquid de-mixing in vitro, when incubated at high concentration with a binding RNA ${ }^{41,42}$. Similarly, MATR3, TDP-43 and CELF1 are components stress granules and paraspeckles, membrane-less RNP granules that form via phase separation of RNA-protein assemblies ${ }^{38-40,43}$. Therefore, we examined whether features that play a role in these 
processes apply to XCI. First, we asked whether rPTBP1 can form liquid-droplets upon interaction with the E-repeat of Xist in vitro. We found that in the absence of RNA, a solution of rPTBP1 $(60 \mathrm{uM})$ remained uniformly clear as assessed by light microscopy (Extended Data Fig. 18a). Addition of the E-repeat RNA at a concentration reported for RNA-PTBP1 droplet formation ${ }^{42}$ $(3.2 \mathrm{uM})$, produced RNA-protein aggregates within an hour (Fig. 5a). Lower RNA concentrations (0.1-0.5uM) produced droplets that resembled phase-separated liquids by multiple parameters: (1) upon addition of RNA, the PTBP1 solution became turbid (data not shown), (2) spherical, transparent droplets accumulated over time, (3) these droplets fused with other droplets, and (4) higher concentrations $(0.3-0.5 \mathrm{uM})$ of E-repeat RNA lead to larger droplets than lower concentrations (0.1uM) (Fig. 5a/b and Extended Data Fig. 18b). In contrast, very small droplets produced by a control RNA, containing 5 short CU-tracts, showed minmal changes in size between upon addition of $0.1-0.5 \mathrm{uM}$ of RNA and did not aggregate at the highest concentration of RNA. These findings indicate that the highly multivalent Xist E-repeat has a high propensity for condensation with PTBP1 ${ }^{59}$.

We also assessed whether self-assembly of TDP-43 affected XCI. TDP-43 forms higherorder complexes that undergo LLPS both in vitro and in vivo ${ }^{60-62}$ and this activity is reduced by mutations: S48E, W334G, W385G and W412G $\mathrm{G}^{60-62}$. If formation of the Xi-domain also involves TDP-43 condensation, a mutant TDP-43 harboring these mutations (TDP-43-4xM, Fig. 4b) should be incapable of rescuing $\Delta \mathrm{E}-X i s t$ associated phenotypes during XCI initiation. Indeed, unlike WT TDP-43 (Fig. 4d), Flag-MCP-TDP-43-4xM did not restore Xist localization or gene silencing upon deletion of the E-repeat (Fig. 5c-d and Extended Data Fig. 19). Similar results were obtained with a MATR3 S85C mutant, previously shown to impair the formation of stress granules that also form from condensation ((Fig. 5c-d and Extended Data Fig. 19) ${ }^{63-66}$. From these data, we posit that by binding these proteins at high density via the E-repeat, Xist effectively concentrates PTBP1, MATR3, TDP-43 and CELF1 to establish a physical condensate, that matures over time during the initiation of XCI and relies on self-aggregation properties to compartmentalize Xist and enforce $\mathrm{X}$-linked gene silencing during the subsequent Xist-independent phase of XCI.

Our results suggested that the condensate set up by the E-repeat could be retained in the absence of Xist at least through a few rounds of cell division, and this could explain how the Xist- 
independent phase of XCI initiation is achieved when Xist is deleted after day 3 of differentiation ${ }^{5}$. To test the possibility that the protein condensate might be retained in the absence of Xist, we immunostained CELF1 in primary female MEFs carrying a conditional Xist allele ${ }^{67}$ (Fig. 5e) to detect the E-repeat dependent condensate. As seen with differentiating ESCs, in the WT MEFs we observed CELF1 enrichment that co-localized with the H3K27me3 accumulation over the Xi (Fig. 5e). We then deleted Xist in the MEF cells using Cre-mediated recombination (Extended Data Fig. 20). As expected, loss of Xist led to loss of $\mathrm{H} 3 \mathrm{~K} 27 \mathrm{me} 3$ accumulation on the $\mathrm{Xi}^{17,26}$ but strikingly, in half of the Xist-deleted cells, CELF1 maintained the punctal enrichment (Fig. 5f). We conclude that the E-repeat-seeded protein condensate is stable without Xist, which likely explains how silencing can be enforced during the Xist-independent phase of XCI.

Our results define a new model for how Xist establishes the Xi-domain during XCI initiation (Fig. $5 \mathrm{~g} / \mathrm{h}$-top). Upon induction of differentiation, Xist is upregulated, assembles with proteins across the RNA (Fig. 5g), and spreads along the X-chromosome ${ }^{24}$ (Fig. 5h, top left). Previous studies have elucidated funtions for several of these proteins. SAF-A/hnRNP-U mediates the chromatin attachment of Xist ${ }^{68}$, while the proteins SHARP ${ }^{25}$, bound at the A-repeat, and PRC1 recruited via hnRNP-K binding to the B-repeat ${ }^{29}$, silence transcription (Fig. $5 \mathrm{~h}$, top middle). We now define a function for the Xist E-repeat that recruits the RNA binding proteins PTBP1, MATR3, TDP-43 and CELF1 during the initiation of XCI. These factors each carry multiple RRMs allowing for the simultaneous engagement of distinct repeat motifs within in the long Erepeat sequence, whose multivalency will increase the avidity of binding to a single transcript (Fig. $5 \mathrm{~h}$, top middle). Our results also define homotypic and heterotypic interactions between these factors required for the faithful initiation of XCI. Together, these multivalent RNA-protein and protein-protein interactions will form a higher-order Xist-protein network. We propose that increasing Xist abundance with differentiation and, likely, compaction of the X-chromosome ${ }^{18}$ concentratrate the Xist binding factors within the confined nuclear region of the $\mathrm{Xi}$ and result in condensation of PTBP1, MATR3, TDP-43 and CELF1 around the nucleating Xist molecules.

After day 3 of differentiation and XCI initiation, the condensate formed by the E-repeat binding proteins is critical for XCI and sustained silencing of X-linked genes during the Xistindependent phase of XCI initiation (Fig. 5h, top right). At this point, the E-repeat has led to 
coalescence of Xist transcripts into the Xist granules and compartmentalization of the Xist-coated $\mathrm{Xi}$. By binding and concentrating the factors needed for establishment of the Xi-domain, Xist enforces its own cis-limited spread and resultant gene silencing. The formation of the E-repeatdependent condensate can allow Xist to remain associated solely on the X chromosome from which it is expressed. In the absence of the E-repeat, the loss of gene silencing and the dissociation of Xist from the X-chromosome domain only occur after trancriptional shutoff and heterochromatin formation (Fig. 5h bottom). We suggest that gene silencing, loss of active transcriptional regulators, and heterochromatinization alter the interaction of Xist with the $\mathrm{Xi}$ and induce a transition from an E-repeat-independent mode of association to one that is E-repeat-dependent.

Our model also suggests a mechanism for the epigenetic memory that perpetuates the silent state after the inducing molecule (Xist) has been deleted. We propose that continued gene silencing upon deletion of Xist after day 3 of differentiation ${ }^{5}$, is mediated by the E-repeat-seeded protein condensate. This is consistent with our finding that CELF1 enrichment on the Xi can be maintained in the absence of Xist. We hypothesize that the condensate integrates additional Xist-interacting proteins, such as SHARP, via specific protein interactions (Fig. 5h top/bottom right). Weak interactions between these different proteins might permit them to diffuse within the Xi-domain. In this way, Xist-interactors, such as SHARP, maintain association with the multi-molecular assembly independently of direct Xist interaction (Fig. 5h top right). Such a model could explain how 100-200 Xist granules (foci) ${ }^{18,52}$ can silence $>1000$ genes across the $167 \mathrm{Mb}$ of $\mathrm{X}$ chromosome DNA. A full understanding of how the condensed silencing domain controls gene silencing will involve determining all of its components and their stoichiometry within the $\mathrm{Xi}$ as well further biophysical characterization of the condenstate. It will be particularly interesting to examine individual genes within the compartment and whether silenced or escaper genes exhibit different interations with the condensate or locations within it.

Our silencing domain model may also explain the finding that Xist-dependent silencing can only be triggered within a defined developmental window upon onset of XCI. PTBP1, MATR3 and CELF1 are highly expressed in ESCs and decline in abundance upon differentiation ${ }^{69}$. In differentiated cells, the lower levels of these RBPs may be insufficient to multimerize on Xist and condense into a silenced compartment. 
Our work provides a new way of thinking about the mechanism of XCI, where the condensation of Xist with its interacting proteins drives the compartmentalization needed for sustained gene regulation. Our results also reveal how RBPs, known for their roles in RNA processing, mediate lncRNA localization and exert control over gene regulation via mechanisms independent of their previously described RNA processing activities. 


\section{References}

1. Brockdorff, N. et al. The product of the mouse Xist gene is a $15 \mathrm{~kb}$ inactive X-specific transcript containing no conserved ORF and located in the nucleus. Cell 71, 515-526 (1992).

2. Brown, C. J. et al. The human XIST gene: analysis of a $17 \mathrm{~kb}$ inactive X-specific RNA that contains conserved repeats and is highly localized within the nucleus. Cell 71, 527-542 (1992).

3. Marahrens, Y., Panning, B., Dausman, J., Strauss, W. \& Jaenisch, R. Xist-deficient mice are defective in dosage compensation but not spermatogenesis. Genes Dev. 11, 156-166 (1997).

4. Penny, G. D., Kay, G. F., Sheardown, S. A., Rastan, S. \& Brockdorff, N. Requirement for Xist in X chromosome inactivation. Nature 379, 131-137 (1996).

5. Wutz, A. \& Jaenisch, R. A shift from reversible to irreversible X inactivation is triggered during ES cell differentiation. Mol. Cell 5, 695-705 (2000).

6. Wutz, A., Rasmussen, T. P. \& Jaenisch, R. Chromosomal silencing and localization are mediated by different domains of Xist RNA. Nat. Genet. 30, 167-174 (2002).

7. Yang, L., Kirby, J. E., Sunwoo, H. \& Lee, J. T. Female mice lacking Xist RNA show partial dosage compensation and survive to term. Genes Dev. 30, 1747-1760 (2016).

8. Robert Finestra, T. \& Gribnau, J. X chromosome inactivation: silencing, topology and reactivation. Curr. Opin. Cell Biol. 46, 54-61 (2017).

9. Galupa, R. \& Heard, E. X-Chromosome Inactivation: A Crossroads Between Chromosome Architecture and Gene Regulation. Annu. Rev. Genet. 52, 535-566 (2018).

10. Brockdorff, N. Local Tandem Repeat Expansion in Xist RNA as a Model for the Functionalisation of ncRNA. Non-Coding RNA 4, (2018).

11. Froberg, J. E., Yang, L. \& Lee, J. T. Guided by RNAs: X-inactivation as a model for lncRNA function. J. Mol. Biol. 425, 3698-3706 (2013).

12. Monfort, A. \& Wutz, A. Progress in understanding the molecular mechanism of Xist RNA function through genetics. Philos. Trans. R. Soc. Lond. B. Biol. Sci. 372, (2017).

13. Monk, M. \& Harper, M. I. Sequential X chromosome inactivation coupled with cellular differentiation in early mouse embryos. Nature 281, 311-313 (1979).

14. Chaumeil, J., Le Baccon, P., Wutz, A. \& Heard, E. A novel role for Xist RNA in the formation of a repressive nuclear compartment into which genes are recruited when silenced. Genes Dev. 20, 2223-2237 (2006).

15. Jonkers, I. et al. Xist RNA Is Confined to the Nuclear Territory of the Silenced X Chromosome throughout the Cell Cycle. Mol. Cell. Biol. 28, 5583-5594 (2008).

16. Costanzi, C. \& Pehrson, J. R. Histone macroH2A1 is concentrated in the inactive X chromosome of female mammals. Nature 393, 599-601 (1998).

17. Plath, K. Role of Histone H3 Lysine 27 Methylation in X Inactivation. Science 300, 131135 (2003).

18. Smeets, D. et al. Three-dimensional super-resolution microscopy of the inactive X chromosome territory reveals a collapse of its active nuclear compartment harboring distinct Xist RNA foci. Epigenetics Chromatin 7, 8 (2014).

19. Teller, K. et al. A top-down analysis of Xa- and Xi-territories reveals differences of higher order structure at $\geq 20 \mathrm{Mb}$ genomic length scales. Nucl. Austin Tex 2, 465-477 (2011). 20. Jégu, T. et al. Xist RNA antagonizes the SWI/SNF chromatin remodeler BRG1 on the inactive X chromosome. Nat. Struct. Mol. Biol. 26, 96-109 (2019). 
21. Chen, C.-K. et al. Xist recruits the X chromosome to the nuclear lamina to enable chromosome-wide silencing. Science 354, 468-472 (2016).

22. Chu, C. et al. Systematic discovery of Xist RNA binding proteins. Cell 161, 404-416 (2015).

23. Deng, X. et al. Bipartite structure of the inactive mouse X chromosome. Genome Biol. 16, $152(2015)$.

24. Engreitz, J. M. et al. The Xist lncRNA exploits three-dimensional genome architecture to spread across the X chromosome. Science 341, 1237973 (2013).

25. McHugh, C. A. et al. The Xist lncRNA interacts directly with SHARP to silence transcription through HDAC3. Nature 521, 232-236 (2015).

26. Zhang, L.-F., Huynh, K. D. \& Lee, J. T. Perinucleolar targeting of the inactive X during S phase: evidence for a role in the maintenance of silencing. Cell 129, 693-706 (2007).

27. Żylicz, J. J. et al. The Implication of Early Chromatin Changes in X Chromosome Inactivation. Cell 176, 182-197.e23 (2019).

28. Nesterova, T. B. et al. Characterization of the genomic Xist locus in rodents reveals conservation of overall gene structure and tandem repeats but rapid evolution of unique sequence. Genome Res. 11, 833-849 (2001).

29. Pintacuda, G. et al. hnRNPK Recruits PCGF3/5-PRC1 to the Xist RNA B-Repeat to Establish Polycomb-Mediated Chromosomal Silencing. Mol. Cell 68, 955-969.e10 (2017). 30. da Rocha, S. T. et al. Jarid2 Is Implicated in the Initial Xist-Induced Targeting of PRC2 to the Inactive X Chromosome. Mol. Cell 53, 301-316 (2014).

31. Sunwoo, H., Colognori, D., Froberg, J. E., Jeon, Y. \& Lee, J. T. Repeat E anchors Xist RNA to the inactive X chromosomal compartment through CDKN1A-interacting protein (CIZ1). Proc. Natl. Acad. Sci. U. S. A. 114, 10654-10659 (2017).

32. Yamada, N. et al. Xist Exon 7 Contributes to the Stable Localization of Xist RNA on the Inactive X-Chromosome. PLoS Genet. 11, e1005430 (2015).

33. Yue, M. et al. Xist RNA repeat $\mathrm{E}$ is essential for ASH2L recruitment to the inactive X and regulates histone modifications and escape gene expression. PLOS Genet. 13, e1006890 (2017).

34. Minajigi, A. et al. Chromosomes. A comprehensive Xist interactome reveals cohesin repulsion and an RNA-directed chromosome conformation. Science 349, (2015).

35. Jeon, Y. \& Lee, J. T. YY1 tethers Xist RNA to the inactive X nucleation center. Cell 146, 119-133 (2011).

36. Patil, D. P. et al. m(6)A RNA methylation promotes XIST-mediated transcriptional repression. Nature 537, 369-373 (2016).

37. Moindrot, B. et al. A Pooled shRNA Screen Identifies Rbm15, Spen, and Wtap as Factors Required for Xist RNA-Mediated Silencing. Cell Rep. 12, 562-572 (2015).

38. Fox, A. H., Nakagawa, S., Hirose, T. \& Bond, C. S. Paraspeckles: Where Long Noncoding RNA Meets Phase Separation. Trends Biochem. Sci. 43, 124-135 (2018).

39. Fujimura, K., Kano, F. \& Murata, M. Dual localization of the RNA binding protein CUGBP-1 to stress granule and perinucleolar compartment. Exp. Cell Res. 314, 543-553 (2008).

40. Kato, M. et al. Cell-free Formation of RNA Granules: Low Complexity Sequence Domains Form Dynamic Fibers within Hydrogels. Cell 149, 753-767 (2012).

41. Li, P. et al. Phase transitions in the assembly of multivalent signalling proteins. Nature 483, 336-340 (2012). 
42. Lin, Y., Protter, D. S. W., Rosen, M. K. \& Parker, R. Formation and Maturation of Phase-Separated Liquid Droplets by RNA-Binding Proteins. Mol. Cell 60, 208-219 (2015). 43. Maharana, S. et al. RNA buffers the phase separation behavior of prion-like RNA binding proteins. Science 360, 918-921 (2018).

44. Coelho, M. B. et al. Nuclear matrix protein Matrin3 regulates alternative splicing and forms overlapping regulatory networks with PTB. EMBO J. 34, 653-668 (2015).

45. Han, A. et al. De novo prediction of PTBP1 binding and splicing targets reveals unexpected features of its RNA recognition and function. PLoS Comput. Biol. 10, e1003442 (2014).

46. Marquis, J. et al. CUG-BP1/CELF1 requires UGU-rich sequences for high-affinity binding. Biochem. J. 400, 291-301 (2006).

47. Vuong, J. K. et al. PTBP1 and PTBP2 Serve Both Specific and Redundant Functions in Neuronal Pre-mRNA Splicing. Cell Rep. 17, 2766-2775 (2016).

48. Buratti, E. Nuclear factor TDP-43 and SR proteins promote in vitro and in vivo CFTR exon 9 skipping. EMBO J. 20, 1774-1784 (2001).

49. Rogelj, B. et al. Widespread binding of FUS along nascent RNA regulates alternative splicing in the brain. Sci. Rep. 2, 603 (2012).

50. Silva, J. et al. Establishment of histone h3 methylation on the inactive X chromosome requires transient recruitment of Eed-Enx1 polycomb group complexes. Dev. Cell 4, 481-495 (2003).

51. Buzin, C. H., Mann, J. R. \& Singer-Sam, J. Quantitative RT-PCR assays show Xist RNA levels are low in mouse female adult tissue, embryos and embryoid bodies. Dev. Camb. Engl. 120, 3529-3536 (1994).

52. Sunwoo, H., Wu, J. Y. \& Lee, J. T. The Xist RNA-PRC2 complex at 20-nm resolution reveals a low Xist stoichiometry and suggests a hit-and-run mechanism in mouse cells. Proc. Natl. Acad. Sci. U. S. A. 112, E4216-4225 (2015).

53. Marks, H. et al. Dynamics of gene silencing during $\mathrm{X}$ inactivation using allele-specific RNA-seq. Genome Biol. 16, 149 (2015).

54. Lee, J. T. \& Lu, N. Targeted mutagenesis of Tsix leads to nonrandom X inactivation. Cell 99, 47-57 (1999).

55. Bertrand, E. et al. Localization of ASH1 mRNA Particles in Living Yeast. Mol. Cell 2, 437-445 (1998).

56. Ridings-Figueroa, R. et al. The nuclear matrix protein CIZ1 facilitates localization of Xist RNA to the inactive X-chromosome territory. Genes Dev. 31, 876-888 (2017).

57. Belgrader, P., Dey, R. \& Berezney, R. Molecular cloning of matrin 3. A 125-kilodalton protein of the nuclear matrix contains an extensive acidic domain. J. Biol. Chem. 266, 98939899 (1991).

58. Robinson, F. \& Smith, C. W. J. A splicing repressor domain in polypyrimidine tractbinding protein. J. Biol. Chem. 281, 800-806 (2006).

59. Banani, S. F., Lee, H. O., Hyman, A. A. \& Rosen, M. K. Biomolecular condensates: organizers of cellular biochemistry. Nat. Rev. Mol. Cell Biol. 18, 285-298 (2017).

60. Gopal, P. P., Nirschl, J. J., Klinman, E. \& Holzbaur, E. L. F. Amyotrophic lateral sclerosis-linked mutations increase the viscosity of liquid-like TDP-43 RNP granules in neurons. Proc. Natl. Acad. Sci. U. S. A. 114, E2466-E2475 (2017). 
61. Li, H.-R., Chiang, W.-C., Chou, P.-C., Wang, W.-J. \& Huang, J.-R. TAR DNA-binding protein 43 (TDP-43) liquid-liquid phase separation is mediated by just a few aromatic residues. J. Biol. Chem. (2018). doi:10.1074/jbc.AC117.001037

62. Wang, A. et al. A single N-terminal phosphomimic disrupts TDP-43 polymerization, phase separation, and RNA splicing. EMBO J. 37, e97452 (2018).

63. Protter, D. S. W. \& Parker, R. Principles and Properties of Stress Granules. Trends Cell Biol. 26, 668-679 (2016).

64. Mensch, A. et al. The p.S85C-mutation in MATR3 impairs stress granule formation in Matrin-3 myopathy. Exp. Neurol. 306, 222-231 (2018).

65. Gallego-Iradi, M. C. et al. N-terminal sequences in matrin 3 mediate phase separation into droplet-like structures that recruit TDP43 variants lacking RNA binding elements. Lab.

Invest. 99, 1030-1040 (2019).

66. Iradi, M. C. G. et al. Characterization of gene regulation and protein interaction networks for Matrin 3 encoding mutations linked to amyotrophic lateral sclerosis and myopathy. Sci. Rep. 8, (2018).

67. Pasque, V. et al. X chromosome reactivation dynamics reveal stages of reprogramming to pluripotency. Cell 159, 1681-1697 (2014).

68. Hasegawa, Y. et al. The matrix protein hnRNP U is required for chromosomal localization of Xist RNA. Dev. Cell 19, 469-476 (2010).

69. Chronis, C. et al. Cooperative Binding of Transcription Factors Orchestrates Reprogramming. Cell 168, 442-459.e20 (2017).

70. Ng, K. et al. A system for imaging the regulatory noncoding Xist RNA in living mouse embryonic stem cells. Mol. Biol. Cell 22, 2634-2645 (2011).

71. Rohland, N. \& Reich, D. Cost-effective, high-throughput DNA sequencing libraries for multiplexed target capture. Genome Res. 22, 939-946 (2012).

72. Cremer, M. et al. Multicolor 3D Fluorescence In Situ Hybridization for Imaging Interphase Chromosomes. in The Nucleus (ed. Hancock, R.) 463, 205-239 (Humana Press, 2012).

73. Henegariu, O., Bray-Ward, P. \& Ward, D. C. Custom fluorescent-nucleotide synthesis as an alternative method for nucleic acid labeling. Nat. Biotechnol. 18, 345-348 (2000).

74. Markaki, Y., Smeets, D., Cremer, M. \& Schermelleh, L. Fluorescence in situ hybridization applications for super-resolution 3D structured illumination microscopy. Methods Mol. Biol. Clifton NJ 950, 43-64 (2013).

75. Kraus, F. et al. Quantitative 3D structured illumination microscopy of nuclear structures. Nat. Protoc. 12, 1011-1028 (2017).

76. Beard, C., Hochedlinger, K., Plath, K., Wutz, A. \& Jaenisch, R. Efficient method to generate single-copy transgenic mice by site-specific integration in embryonic stem cells. Genes. N. Y. N 2000 44, 23-28 (2006).

77. Pandya-Jones, A. \& Black, D. L. Co-transcriptional splicing of constitutive and alternative exons. RNA N. Y. N 15, 1896-1908 (2009).

78. Davidovich, C., Zheng, L., Goodrich, K. J. \& Cech, T. R. Promiscuous RNA binding by Polycomb repressive complex 2. Nat. Struct. Mol. Biol. 20, 1250-1257 (2013).

79. Damianov, A. et al. Rbfox Proteins Regulate Splicing as Part of a Large Multiprotein Complex LASR. Cell 165, 606-619 (2016).

80. Van Nostrand, E. L. et al. Robust transcriptome-wide discovery of RNA-binding protein binding sites with enhanced CLIP (eCLIP). Nat. Methods 13, 508-514 (2016). 
81. Langmead, B., Trapnell, C., Pop, M. \& Salzberg, S. L. Ultrafast and memory-efficient alignment of short DNA sequences to the human genome. Genome Biol. 10, R25 (2009).

82. Trapnell, C., Pachter, L. \& Salzberg, S. L. TopHat: discovering splice junctions with RNA-Seq. Bioinforma. Oxf. Engl. 25, 1105-1111 (2009).

83. Yeo, G. CLIPper. A tool to define peaks in your CLIP-seq dataset.

84. Karperian, A, A. FracLac for ImageJ. (1999).

85. Demmerle, J. et al. Strategic and practical guidelines for successful structured illumination microscopy. Nat. Protoc. 12, 988-1010 (2017).

86. Schindelin, J. et al. Fiji: an open-source platform for biological-image analysis. Nat. Methods 9, 676-682 (2012).

87. Schneider, C. A., Rasband, W. S. \& Eliceiri, K. W. NIH Image to ImageJ: 25 years of image analysis. Nat. Methods 9, 671-675 (2012).

88. Ollion, J., Cochennec, J., Loll, F., Escudé, C. \& Boudier, T. TANGO: a generic tool for high-throughput 3D image analysis for studying nuclear organization. Bioinformatics 29, 18401841 (2013). 


\section{Author contributions}

K.P., A.P-J, Y.M., and D.L.B conceptualized the project, A.P-J., Y.M., J.S., T.C., W.M., A.D., B.P., K.C., R.M., X-J.W., C-K.C., and A.C. acquired data, A.P-J., J.S., Y.M., T.C., H.L. and K.P., analyzed data, A.P-J, Y.M., J.S., M.G. and K.P. interpreted the data and contributed towards methodology and model creation, K.P., D.L.B, M.G. and H.L. acquired funding to support the project, A.P-J. and K.P. administered the project and A.P-J. and K.P. wrote the manuscript and included edits from all authors.

\section{Author Information}

Authors declare no competing interests.

Correspondence and requests for materials should be addressed to kplath@mednet.ucla.edu.

\section{Data and materials availability}

All genomic data for Xist interactions and chromatin association will be deposited in the Gene Expression Omnibus (GEO) database before publication of the manuscript. All reagents will be made available upon request after the manuscripts has been accepted for publication. 


\section{Materials and Methods}

\section{Cell Culture}

All mouse ESC lines were cultured in knockout DMEM (Life Technologies) supplemented with 15\% FBS (Omega), 2 mM L-glutamine (Life Technologies), 1× NEAA (Life Technologies), 0.1 $\mathrm{mM}$ Beta-Mercaptoethanol (Sigma), 1xPenicillin/Streptomycin (Life Technologies), and 1000 $\mathrm{U} / \mathrm{mL}$ murine LIF (homemade) on $0.3 \%$ gelatinized plates (porcine skin gelatin, Sigma) pre-plated with irradiated male DR4 feeders (homemade from day 14.5 embryos with appropriate animal protocols in place). For 3D-SIM microscopy experiments (see below), ESCs were maintained in $2 \mathrm{i}$ culture conditions. No differences in results upon cell differentiation (see below) were observed between the ESC propagation conditions. ESCs were maintained as small colonies and passaged with trypsin and single cell dissociation at $80 \%$ confluency.

\section{Female ESC Differentiation}

Female WT F1 2-1 MS2 $2^{12970}$ (and derivatives thereof) were trypsinized to single cells and counted. Cells were seeded in $2 \mathrm{~mL}$ of mouse embryonic fibroblast (MEF) medium [DMEM (Invitrogen) supplemented with 10\% FBS (Omega), 2 mM L-glutamine (Life Technologies), 1× NEAA (Life Technologies), $0.1 \mathrm{mM}$ Beta-Mercaptoethanol (Sigma) and 1xPenicillin/Streptomycin (Life Technologies)] at a density of 20,000 - 200,000 cells $/ 4 \mathrm{~cm}^{2}$ (depending on the experiment) on tissue culture plates for Western Blotting or onto $18 \mathrm{~mm}$ sterile glass coverslips for IF/FISH experiments, both of which were pre-coated with sterile $0.3 \%$ gelatin (porcine skin gelatin, sigma) or matrigel (Corning)(diluted 1:100). 24 hours post-seeding, the culture medium was changed and supplemented with $1 \mu \mathrm{M}$ all-trans Retinoic Acid (Sigma), which was changed daily thereafter until the cells were harvested for analysis.

\section{Female MEF culture}

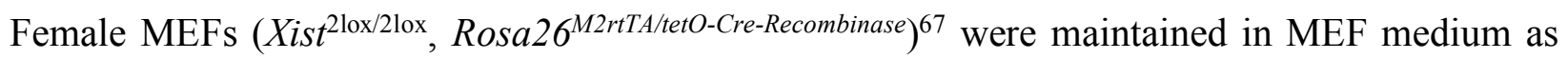
described above. To delete Xist, cells were treated with $2 \mu \mathrm{g} / \mathrm{mL}$ doxycycline (sigma) for $96 \mathrm{~h}$.

\section{RNA Fluorescence In Situ Hybridization}

FISH against Xist RNA was performed using both RNA and DNA probes. FISH against MS2, Atrx, Gpc4, Mecp2, Rnf12 and Chic1 was performed using DNA probes. In undifferentiated ESCs, the DNA probe against Xist additionally detects Tsix. 
RNA Probe Preparation: Strand-specific RNA probes were generated using a T3 in vitro transcription kit (Promega) in the presence of Chromatide AlexaFluor-UTP (ThermoFisher). Six 700nt transcription templates were generated from Xist exon 1 (Primers UCLA 1416 - 1429, Extended Data Table 1), and used in transcription reactions containing 0.5mM ATP, CTP, GTP, 0.1mM UTP, and 0.05mM Chromatide AlexaFluor488-UTP (Life Technologies) along with 1x T3 transcription buffer supplemented with 10mM DTT, 500U RNase inhibitor, 170U T3 RNA polymerase and $5 \mu \mathrm{g}$ of pooled template DNA in a final volume of $500 \mu \mathrm{L}$ at $37^{\circ} \mathrm{C}$ overnight in the dark. The transcription reaction was treated with $15 \mathrm{U}$ RNase-Free DNase for 15 minutes at $37^{\circ} \mathrm{C}$ prior to probe purification. To purify the probes, $1 / 3$ of the transcription reaction was loaded on a pre-spun (700xg, 5minutes) Chromaspin-100 column (Clontech) and centrifuged (700g, 5 minutes). The eluates were combined and precipitated with $100 \% \mathrm{EtOH}$ in the presence of $100 \mathrm{mg}$ tRNA and $1 / 10^{\text {th }}$ volume of sodium acetate (Sigma). We sometimes also purified RNA probes using a 2.5x volume of AMPure beads (ThermoFisher 09-981-123, reconstituted according to ${ }^{71}$ ) , which were washed twice on a magnet with $80 \%$ ethanol before elution of the probes from the beads with $50 \mu \mathrm{L}$ water, followed by ethanol precipitation. The RNA pellet was washed twice in $70 \%$ ethanol, resuspended in $400 \mu \mathrm{L}$ of RNase Free water, to which $1 \mathrm{~mL}$ EtOH was added for storage at $-20 \mathrm{C}$. To make the final probe, $1 / 7^{\text {th }}$ of the Probe/EtOH solution was added to $90 \mu \mathrm{L}$ Salmon Sperm DNA (Sigma), 90 $\mu$ L mouse Cot1 DNA (Life Technologies), 40 $\mu$ L 3M RNase Free Sodium Acetate (Sigma), 40 $\mu \mathrm{L}$ 10mg/mL tRNA (Life Technologies) and 1mL EtOH. After vigorous shaking, the solution was centrifuged at maximum speed for 10 minutes. The pellet was washed once with $70 \% \mathrm{EtOH}$ and then once with 100\% EtOH, allowed to dry completely, and then resuspended in $200 \mu \mathrm{L}$ deionized Formamide (VWR) and $200 \mu \mathrm{L} 2 \mathrm{x}$ Hybridization buffer [20\% dextran sulfate (sigma), $4 \times$ SSC (Ambion), $\left.0.1 \mathrm{M} \mathrm{NaH}_{2} \mathrm{PO}_{4}\right]$. Probes were stored at $-80 \mathrm{C}$ and denatured at $95^{\circ} \mathrm{C}$ for 5 minutes before use.

DNA probe preparation: For 3D-SIM and Airyscan experiments, FISH probes were labelled by nick translation as described in ${ }^{72}$, using p15 cDNA plasmid as template and home-labelled Atto488-, Cy3- or Texas Red-conjugated dUTPs made as described in ${ }^{73}$. For all other experiments, DNA probes were synthesized using the CGH Bioprime Array Kit (ThermoFisher) 
according to manufacturer's instructions. Briefly, a $40 \mu \mathrm{L}$ solution containing $100 \mathrm{ng}$ of template plasmid DNA was denatured in the presence of $1 \mathrm{x}$ random primers at $95^{\circ} \mathrm{C}$ for 5 minutes and snap cooled on ice. $5 \mu \mathrm{L}$ of nucleotide mix, $5 \mu \mathrm{L}$ of 488 , 555-, or $594-\mathrm{dUTP}$ or dCTP chromatide fluorphore (Life Technologies) and 5U Klenow exo- enzyme were then added and incubated in the dark at $37^{\circ} \mathrm{C}$ for 6 hours, after which an additional $5 \mathrm{U}$ of Klenow Exo- were added. The reaction was incubated at $37^{\circ} \mathrm{C}$ overnight, quenched with $10 \mu \mathrm{L}$ stop solution, and then purified over a Chromatide-100 column or AMPure beads as described above. The eluate was precipitated in the presence of 100mg yeast tRNA (Life Technologies) and sodium acetate (Sigma). The DNA probe was then prepared as above to yield $400 \mu \mathrm{L}$ of probe solution in formamide/hybridization buffer.

The MS2 DNA template for DNA probe preparation was PCR amplified from genomic DNA purified from WT F1 2-1 MS2 ${ }^{129}$ female ESCs (see Extended Data Table 1 for primers). For Xist, the DNA probe was synthesized using a full-length mouse Xist cDNA plasmid (p15A-31-17.9kb Xist, unpublished). Probes against X-linked genes were synthesized using BACs RP23-467J21 (Gpc4), RP23-265D6 (Atrx), WIBR1-2150D22 (Chic1), WIBR1-2704K12 (Rnf12) and W11894A5 and W11-1189K18 (Mecp2) (all obtained from CHORI-BACPAC). Note that the use of 2 BAC's for the Mecp2 probe sometimes resulted nascent FISH signals that appeared as doublets (see Fig. 3b).

RNA FISH procedure for Epifluorescence microscopy: Culture medium was changed 10 minutes prior to harvesting cells to remove dead cells and stimulate transcription. Upon collection, culture medium was aspirated and coverslips were gently rinsed twice with cold 1xPBS. Coverslips were then transferred to a new culture dish containing cold 1xPBS, which was then aspirated, and the cells were fixed in 4\% paraformaldehyde (PFA) (Electron Microscopy Sciences) in 1× PBS for 10 minutes at room temperature (RT) under standard laboratory safety practices. After fixation, the cells were permeabilized in $0.5 \%$ Triton X-100 (Acros) in 1xPBS with 2mM Vanadyl Ribonucleoside Complex (NEB) for 10-20 minutes on ice. Coverslips were then stored in 70\% ethanol at $-20^{\circ} \mathrm{C}$ for 1 hour or until samples from all time points had been collected. Prior to hybridization with probe, the coverslips with cells were brought back to $4^{\circ} \mathrm{C}$ and serially 
dehydrated by 5-minute incubations in ice-cold $80 \%, 95 \%$ and $100 \%$ ethanol. Coverslips were removed from $100 \%$ ethanol and allowed to air dry prior to incubation with probe for 48 hours at $37^{\circ} \mathrm{C}$ in a sealed chamber humidified with $2 \mathrm{xSSC} / 50 \%$ Formamide. For RNA probes, coverslips were washed $3 \times 5$ minutes in 50\% formamide (Fisher) / 2xSSC (Ambion) and 3x 5 minutes in Wash Buffer II (10mM Tris, $0.5 \mathrm{M} \mathrm{NaCl}, 0.1 \%$ Tween-20), prior to a 45 minutes incubation with $25 \mu \mathrm{g} / \mathrm{mL}$ RNaseA (ThermoFisher) in Wash Buffer II at $37^{\circ} \mathrm{C}$. After RNaseA treatment, coverslips were washed 2x 5 minutes in Wash Buffer II, 2x 5 minutes in 50\% formamide / $2 \times$ SSC, $3 \times 5$ minutes in $2 \times \mathrm{SSC}$ and $3 \times 5$ minutes in $1 \times \mathrm{SSC}$ before briefly drying excess $1 \times \mathrm{SSC}$ off and mounting with Vectashield mounting media lacking DAPI (Vector Labs). Coverslips were sealed with Biotium Covergrip coverslip sealant (Thermo Fisher). For DNA probes, coverslips were washed $3 \times 5$ minutes in 50\% Formamide / 2xSSC, 3x 5 minutes in 2xSSC and 3x 5 minutes in 1xSSC prior to mounting. A $1: 10,000$ dilution of DAPI $(0.5 \mathrm{mg} / \mathrm{mL})$ was included in all penultimate $1 \mathrm{xSSC}$ washes. All washes were conducted at $42^{\circ} \mathrm{C}$, cells were protected from light. All procedures were performed, and used reagents disposed of, according to standard laboratory safety procedures.

RNA FISH procedure for 3D-SIM and Improved-Resolution microscopy: All coverslips were processed according to ${ }^{74}$.

\section{Indirect Immunofluorescence staining}

The cell culture medium was changed 10 minutes prior to harvesting. Upon collection, culture medium was aspirated and coverslips were gently rinsed twice with cold 1xPBS. Coverslips were then transferred to a new culture dish containing cold 1xPBS. If cells were CSK treated (MS2-CPGFP expressing wild-type WT F1 2-1 MS2 ${ }^{129}$ ESCs), then coverslips were gently treated with $1 \mathrm{~mL}$ (added dropwise) ice-cold CSK buffer [100mM NaCl, $300 \mathrm{mM}$ sucrose, $3 \mathrm{mM} \mathrm{MgCl}, 10 \mathrm{mM}$ PIPES $\mathrm{pH}$ 6.8] and incubated on ice for 30 seconds before aspiration. Coverslips were then similarly treated with $1 \mathrm{~mL}$ ice-cold CSK-Trt Buffer $(\mathrm{CSK}+0.5 \%$ Triton X-100) for 30 seconds, followed with a second ice-cold CSK treatment. Coverslips were then processed as described in 75. See Extended Data Table 2 for antibody information. 


\section{Indirect Immunofluorescence staining -RNA Fluorescence In Situ Hybridization}

Where immunostaining and FISH were combined, immunostaining preceded FISH.

Epifluorescence Microscopy: The immunostaining protocol was followed as outlined above, but coverslips were not mounted. Instead, after the last round of washes (omitting DAPI in the penultimate wash), coverslips were re-fixed in 4\% PFA in $1 \times$ PBS for 10 minutes at room temperature and then dehydrated through a 70-85-95-100\% ice-cold ethanol series prior to overnight incubation with probe as described above in the RNA FISH procedure section.

3D-SIM and Improved-Resolution microscopy: All coverslips were processed according to ${ }^{74}$.

\section{Plasmid Construction and Cell Line Generation}

Xist- $\Delta E$ targeting construct: To create the targeting vector pCR2.1-Puro-Xist $\Delta \mathrm{E}, 3 \mathrm{~kb}$ upstream and $1.2 \mathrm{~kb}$ downstream of the mouse Xist E repeat were PCR amplified from mouse genomic DNA using primers WRM163-166, modified for In-Fusion cloning (Clontech) using Kapa polymerase (Kapa biosystems) according to the manufacturer's instructions. The upstream homology arm was integrated at the EcoR 1 site and the downstream homology arm at the BamH1 site, in a 4-piece InFusion cloning reaction, into a vector containing a floxed puromycin resistance cassette (PCR2.1-loxP-pGK-Puro-pA-loxP). Positive recombinants were identified by restriction digest of with HindIII.

E-repeat deletion in WT F1 2-1 MS2 ${ }^{129}$ ESCs: The Xist E repeat was deleted in female WT F1 2$1 \mathrm{MS} 2^{129} \mathrm{ESCs}$ derived from an F1 cross of mice from pure bread 129 and castaneous background, and then targeted to contain a $11 \mathrm{x}$ tandem repeat of the MS2 hairpin located $1.2 \mathrm{~kb}$ downstream of the E-Repeat ${ }^{70}$ via homologous recombination. The cells also harbor an M2-reverse tetracycline TransActivator cassette within the Rosa26 locus that confers neomcyin resistance on the cells. $1 / 2$ of a confluent T75 flask of WT F1 2-1 MS2 ${ }^{129}$ female ESCs were electroporated with 40 $\mu \mathrm{g}$ of PciI 
linearized PCR2.1-Puro Xist $\Delta \mathrm{E}$ targeting plasmid $(800 \mathrm{v}, 0.2 \mathrm{~ms}, 4 \mathrm{~mm}$ cuvette, Biorad X-Cell electroporation module) and plated at varying dilutions on $10 \mathrm{~cm}$ plates of confluent irradiated DR4 feeders. 36 hours after plating, the cells were selected with $1 \mu \mathrm{g} / \mathrm{mL}$ puromycin for 10 days. 100 clones were picked, expanded and subjected to southern blot analysis using a Sac1 digest and an external probe (amplified using primers WRM193/194 (Extended Data Table 1)) as outlined in Extended Data Fig.5. The positive clone \#35 was expanded in culture, then transfected with a Crerecombinase plasmid using Lipofectamine 2000 according to the manufacturer's protocol (Thermo Fisher), to delete the puromycin resistance cassette. Transfected cells were serially diluted, 100 clones were picked, expanded and replica plated for growth in the presence or absence of puromycin. Sub-clone \#96 was sensitive to puromycin. PCR analysis of genomic DNA confirmed the deletion of the puromycin cassette with primers APJ439/440 (Extended Data Table 1). Subsequent southern blot analysis and sequencing of wild-type Xist and $\triangle \mathrm{E}$ Xist PCR amplicons from genomic DNA (Intron 6 to Exon 7 using APJ248/631 (Extended Data Table 1)) showed that the $\Delta \mathrm{E}$ targeting construct integrated on the 129 allele of Xist upstream of the MS2 tag, preserving the 3' splice site of intron 6, to yield the heterozygous E-repeat deletion ESC line $\mathrm{X}_{129}{ }^{\text {Xist }} \Delta \mathrm{E}$,MS2 $\mathrm{X}_{\text {Cas }}{ }^{\text {Xist } \mathrm{WT}}$ (= $=\mathrm{E}$ ESCs) (Extended Data Fig. 5/6 and data not shown). Sequencing of the exon 6 exon 7 RT-PCR amplicon (obtained from cDNA of differentiated ESCs) derived from the $129^{\mathrm{MS} 2}$ Xist transcript, revealed the use of a cryptic 3' splice site downstream of the LoxP site (Extended Data Fig. 6). This extended the E-repeat deletion within the Xist transcript (as initially designed) by $42 \mathrm{nt}$ and removed the LoxP site and additional vector sequences present in the genomic DNA from mature $\Delta \mathrm{E}$ Xist transcripts, resulting in a scar-less ligation of the 3 ' terminus of exon 6 to nucleotide 1479 of Exon 7 (Extended Data Fig. 6 and data not shown). We ensured that $\triangle$ E ESCs maintained two $\mathrm{X}$ chromosomes throughout the targeting process, and differentiated equally to wild-type as judged by changes in morphology, loss of NANOG upon induction of differentiation (Extended Data Fig. 5).

Engineering of $W T$ and $\triangle \mathrm{E}$ ESCs with a FLP-FRT recombination platform for rescue experiments: WT F1 2-1 MS2 ${ }^{129}$ ESCs and $\Delta \mathrm{E}$ ESCs described above ( $1 / 2$ of a confluent T75 flask) were electroporated with $40 \mu \mathrm{g}$ of Fsp1 linearized Flp-IN homing plasmid that integrates a FRT landing site downstream of the Col1A locus and carried a puromycin resistance cassette for 
targeting ${ }^{76}$, at $800 \mathrm{v}, 0.2 \mathrm{~ms}, 4 \mathrm{~mm}$ cuvette using a Biorad X-Cell electroporation module before being serially diluted on $10 \mathrm{~cm}$ plates, pre-coated with irradiated DR4 feeders. 36 hours after plating, the cells were selected with $2 \mu \mathrm{g} / \mathrm{mL}$ puromycin for 10 days after which 200 clones were picked and expanded. Genomic DNA was isolated and EcoRI digested, before being subjected to southern analysis with the Col1A Xba/Pst1 3' probe. Positive clones 1-61 (WT) and $137(\Delta \mathrm{E})$ were used for all subsequent experiments.

Generation of Flp-In plasmids encoding Flag-MS2-CP fusion proteins: The MS2 Coat Protein (CP) coding sequence was PCR amplified with a forward primer encoding a 3xFlag tag downstream of a Kozak-ATG start signal. The reverse primer contained an in frame Nhe1 site (primers APJ526/570 (Extended Data Table 1)) such that any fragment ligated into the site would be expressed in frame with the MS2-CP protein, separated by a 3-amino acid (Gly-Leu-Gly) linker. The Flag-MS2-CP-Nhe1 fragment was inserted into the EcoRI site of the pBS32 vector using Infusion cloning. This vector is similar to the pgkATGfrt vector described in $^{76}$, except that the tetinducible promoter was replaced with a CAGGS promoter, allowing constitutive expression of the fusion protein. The coding sequence for each protein (GFP, PTBP1, MATR3, TDP-43, and CELF1) fused to the Flag-MS2-CP was PCR amplified from cDNA with infusion overhangs, or synthesized (see below, Genewiz), and ligated into the Nhe1 site of the pBS32-Flag-MS2CP parent plasmid using InFusion cloning (Clontech). The PTBP1 Y247Q, MATR3mutPRI and MATR3 $\triangle$ Zfn mutants were generated using primer-directed mutagenesis. The WT PRI sequence (GILGPPP) was mutated to create the mutant PRI sequence (GAAAPPA) ${ }^{44}$. The coding sequences for the CELF1, MATR3 S85C, TDP43 4xM and MS2CP-GFP-MS2CP fusions were synthesized (Genewiz). All plasmids were verified by sequencing.

The $\Delta \mathrm{C}$-Terminal PTBP1 fragment that is fused to Flag-MS2-CP in our rescue system, is comprised of the first 299 amino acids of PTBP1, which includes the first two RRMs as well as the MATR3 interaction site, followed by 68 amino acids that are out of frame, and do not encode a functional linker region. A premature stop codon terminates the protein at residue 367 . 
Generating WT and $\triangle E$ ESCs expressing Flag-MS2-CP-fusions via Flp-In recombination: $33 \mu \mathrm{g}$

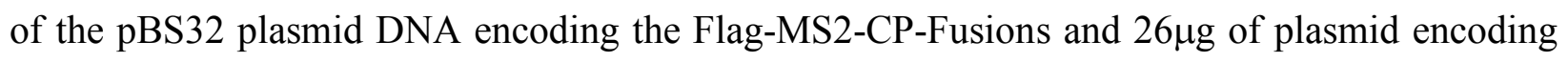
the flpase FlpO were electroporated into WT ESCs carrying the FRT homing site (clone 1-61) for the GFP fusion and $\triangle \mathrm{E}$ ESCs with the FRT homing site (clone 137) for all other fusion constructs (1/2 of a confluent T75 flask of ESCs per electroporation). Cells were plated on confluent irradiated DR4 feeders in a $10 \mathrm{~cm}$ dish and 36 hours after plating selected with $170 \mu \mathrm{g} / \mathrm{mL}$ hygromycin for 14 days, after which all colonies were picked and expanded. The resulting clones were tested for protein expression by immunoblot of lysates (RIPA buffer in 1X SDS lysis buffer (ThermoFisher)) using an anti-Flag antibody as well as antibodies against the respective fusion protein (Extended Data Table 2). Immunostaining confirmed nuclear localization of all fusion proteins that failed to rescue the phenotypes associated with loss of the E-Repeat. All clones used maintained two X chromosomes, as determined by FISH against $T$ six in undifferentiated cells. For all rescue experiments, at least two clones were analyzed, which revealed that the data are robust. Therefore, in most cases only the results from one rescue clone per protein or mutant are shown.

Generation of tet-inducible Xist 4 Tsix V6.5 male ESCs: Tet-On Xist male V6.5 ESCs carrying a tet-inducible promoter in place of the endogenous Xist promoter and a M2rtTA trans-activator as well as puromycin resistance in the R26 locus ${ }^{24}$ ( $1 / 2$ of a confluent T75 flask) were electroporated with $40 \mu \mathrm{g}$ of Not 1 linearized paa2 $\Delta 1.7$ plasmid DNA $(800 \mathrm{v}, 0.2 \mathrm{~ms}, 4 \mathrm{~mm}$ cuvette using a Biorad X-Cell electroporation machine) and plated on confluent irradiated DR4 feeders. 36 hours after plating, the cells were selected with Neomycin/G418 for 10 days after which 100 clones were picked and subjected to southern analysis described in ${ }^{24}$ (data not shown). Positive clone 70 was used for the PTBP1 ChIP-seq experiments.

\section{siRNA treatments}

Silencer Select siRNAs (Thermo Fisher) against PTBP1, MATR3, CELF1, TDP-43 and SAF-A were diluted to $20 \mathrm{nM}$ in 1x siRNA buffer (60mM KCl, 6mM HEPES pH $7.50 .2 \mathrm{mM} \mathrm{MgCl}_{2}$ ), aliquoted and stored at $-80 \mathrm{C}$ until further use. Under sterile conditions at room temperature, $2.5 \mu \mathrm{L}$ of $20 \mathrm{nM}$ siRNA were added to $80 \mu \mathrm{L}$ of fresh Opti-MEM solution (Gibco). 1.6 $\mu \mathrm{L}$ siRNA MAX 
transfection reagent (Life Technologies) were added to $80 \mu \mathrm{L}$ Opti-MEM solution and subsequently added to the siRNA/opti-MEM solution after 5 minutes of incubation. The resulting solution was mixed by pipetting and left to incubate at RT for 20 minutes. The solution was then added to 200,000 cells in $0.8 \mathrm{~mL}$ of culture medium and plated in 1 well of a 12 well plate on $18 \mathrm{~mm}$ gelatinized coverslips and left overnight at 37C. For female ESCs undergoing differentiation, cells were plated in MEF medium and after 24h, the culture medium was changed (with the addition of $1 \mu \mathrm{M}$ all-trans Retinoic Acid)(Sigma) and a second round of siRNA treatment was performed. Knockdown efficiency was assessed by immunoblotting (Extended Data Table 2).

\section{Immunoblotting}

Cells were harvested by trypsinization, pelleted $(1000 \mathrm{xg}, 5 \mathrm{~min})$, resuspended in $500 \mu \mathrm{L} 1 \mathrm{xPBS}$ to wash, and re-pelleted. The washed cell pellet was lysed in 5 pellet volumes of RIPA buffer and 40U benzonase (Novogen) and incubated at 4C overnight. The lysate was centrifuged at max speed to pellet the remaining insoluble material and the supernatant was transferred to a new tube and mixed with $4 \mathrm{x}$ Novex sample buffer containing 5\% 14.3M Beta-mercaptoethanol (Sigma) to a final concentration of $1 \mathrm{x}$. The samples were then denatured for $5 \mathrm{~min}$ at $95 \mathrm{C}$ and loaded onto a 4$12 \%$ Novex Bis-Tris acrylamide gel with 1x MES running buffer (Life Technologies) run at 120V for $1.5-2 \mathrm{~h}$. The gels were transferred to a protran BA-85 nitrocellulose membrane (Whatman) using a Novex XCell II transfer system for $1 \mathrm{~h}$ at $30 \mathrm{~V}, 4 \mathrm{C}$ (or overnight at $4 \mathrm{C}$ at $10 \mathrm{~V}$ ) in transfer buffer (25mM Tris-HCl, 192mM glycine, 20\% methanol). Membranes were probed with primary antibody (Extended Data Table 2) in 1x Odyssey blocking buffer (LI-COR) overnight at 4C, washed $3 \times 5$ minutes in PBS $+0.2 \%$ tween-20 and then incubated with appropriate secondary antibodies (1:10,000 dilution, Odyssey 700 and 800nm antibodies) in the dark at room temperature for 30 minutes before being washed again and scanned on a LI-COR infrared imaging system.

\section{Co-Immunoprecipitation}

For co-immunoprecipitation experiments, Rabbit IgG and antibodies against PTBP1, MATR3, CELF1, CIZ1, TDP-43 (Extended Data Table 2) were crosslinked to ProteinG-Dynabeads (ThermoFisher) using the protocol provided by Abcam (http://www.abcam.com/protocols/cross- 
linking-antibodies-to-beads-protocol) with minor modifications. Briefly, $20 \mu \mathrm{L}$ of bead slurry were isolated on a magnet and washed $3 \times 5$ minutes at room temp in 5 volumes of 1xPBS. Beads were then washed once in 5 volumes of binding buffer $(100 \mu \mathrm{L}, 1 \times \mathrm{xPS}$ containing $1 \mathrm{mg} / \mathrm{mL}$ of BSA (NEB)) for 10 minutes at RT, and incubated in $100 \mu \mathrm{L}$ binding buffer supplemented with $5 \mu \mathrm{g}$ of Rabbit IgG or antibodies against PTBP1, MATR3, CELF1, CIZ1 or TDP43. Samples were rotated for 1 hour at $4^{\circ} \mathrm{C}$. Beads were then washed in binding buffer for 5 minutes, followed by an additional 5 minute wash in 1xPBS. Next, the antibody was crosslinked by incubating in a $100 \mu \mathrm{L}$ of $1 \times$ PBS solution containing $0.2 \mathrm{M}$ triethanolamine (Sigma) and $6.5 \mathrm{mg} / \mathrm{mL}$ Dimethyl pimelimidate (DMP) (Sigma) pH 8.5 for 30 minutes with rotation at RT. Beads were then washed in $250 \mu \mathrm{L} 0.2 \mathrm{M}$ triethanolamine in $1 \mathrm{xPBS}$ for 5 minutes. DMP incubation and wash steps were repeated two more times before samples were quenched in $100 \mu \mathrm{L}$ of $50 \mathrm{mM}$ ethanolamine in 1xPBS for 5 minutes. The quenching step was repeated and excess non-crosslinked antibody removed with 2x 10 minutes incubations in fresh $1 \mathrm{M}$ glycine $\mathrm{pH}$ 3.0. Beads were washed in 1xPBS $3 x 5$ minutes before use in immunoprecipitations. Immunoprecipitations were performed under non-denaturing conditions according to the Abcam protocol (http://www.abcam.com/ps/pdf/protocols/immunoprecipitation\%20protocol\%20(ip).pdf).

$4 \times 15 \mathrm{~cm}$ plates of confluent WT F1 2-1 MS2 ${ }^{129}$ female ESCs were lysed by pipetting in $3 \mathrm{~mL}$ of lysis buffer (10M Tris- $\mathrm{HCl} \mathrm{pH} 8,137 \mathrm{mM} \mathrm{NaCl}, 1 \%$ NP40, 2mM EDTA) supplemented with 1x Complete EDTA-free Protease Inhibitors (Roche) and incubated for $1 \mathrm{~h}$ on ice with or without RNase $\left(10 \mu \mathrm{g} / \mathrm{mL}\right.$ RNase A) (Thermo Fisher). Lysate was centrifuged at $4^{\circ} \mathrm{C}, 14,000 \mathrm{rpm}$ in a tabletop microfuge for 15 minutes to pellet insoluble material. The supernatant was transferred to new tubes and precleared with $20 \mu \mathrm{L}$ of washed ProteinG-dynabeads per $1 \mathrm{~mL}$ of lysate with rotation at $4^{\circ} \mathrm{C}$ for 1 hour. $500 \mu \mathrm{L}$ of lysate were then added to each crosslinked antibody-proteinG Dynabead prep (described above) and rotated at $4^{\circ} \mathrm{C}$ overnight. The next day, crosslinked antibody-proteinG Dynabeads were isolated on a magnet and washed 4x 5 minutes in ice-cold wash buffer (10mM Tris-HCl pH 7.4, 1mM EDTA, 1mM EGTA, 150mM NaCl, 1\% TritonX-100) supplemented with 1x Complete EDTA-free Protease Inhibitors. The co-purified proteins were eluted by boiling in 1x NuPage Protein Loading buffer (Thermo Fisher) supplemented with 5\% Beta-mercaptoethanol, at $95^{\circ} \mathrm{C}$ for 5 minutes. Samples were assessed by immunoblotting. Input represents $4 \%$ of lysate added per immunoprecipitate. $1 / 4^{\text {th }}$ of eluate was loaded per lane. 


\section{In Vitro RNA transcription (IVT)}

For several in vitro experiments (Droplet assays, EMSA), RNAs encoding the E-repeat and other sequences were obtained by IVT. Templates for IVT were amplified from DNA using KAPA polymerase according to manufacturer's instructions (KAPA Biosystems), and then gel purified and concentrated over AMPure beads (homemade). See Extended Data Table 1 for primer information. RNA was transcribed and UREA-PAGE purified as described in ${ }^{77}$. For biotinylated RNAs, Biotin-UTP (Ambion) comprised $18 \%$ of the total UTP.

\section{Droplet Assays}

Recombinant 6x-His tagged PTB was purified using Ni-NTA agarose (Invitrogen) according to manufacturer's instructions. The purified protein was dialyzed and stored in Buffer DG (20 mM HEPES-KOH pH 7.9, 80 mM K. glutamate, 20\% glycerol, $2.2 \mathrm{mM} \mathrm{MgCl}_{2}, 1 \mathrm{mM}$ DTT, and 0.1 mM PMSF). $10 \mu \mathrm{L}$ droplets were assembled in $1.5 \mathrm{~mL}$ Eppendorf tubes as described in ${ }^{42}$. Briefly, $5 \mu \mathrm{L}$ of a $2 \mathrm{x}$ buffer containing $200 \mathrm{mM} \mathrm{NaCl}, 40 \mathrm{mM}$ Imidazole, $2 \mathrm{mM}$ DTT and $20 \%$ glycerol was supplemented with the E-Repeat or control IVT RNA (varying concentrations), PTBP1 (to a final concentration of $60 \mu \mathrm{M}$ ) and water to $10 \mu \mathrm{L}$ (final volume). The solution was mixed by pipetting and transferred to one well of an 8 well chamber slide (Ibidi) that had been pre-coated with 3\% BSA, washed 3x with RNase-Free water and dried. Droplets were imaged at 10x magnification.

\section{Electrophoretic Mobility Shift Assays}

EMSAs were performed as described in ${ }^{78}$ except that $40,000 \mathrm{cpm}$ of 5 ' end labeled RNA were used per condition.

\section{Quantitative RT-PCR and ActinomycinD treatment}

In several experiments we determined the levels of Xist by RT-PCR. For experiments with ActinomcyinD treatment, the drug was dissolved in DMSO at $1 \mathrm{mg} / \mathrm{mL}$ and added to the culture medium to a final concentration of $1 \mu \mathrm{g} / \mathrm{mL}$. For RT-PCR, cells were harvested in $1 \mathrm{~mL}$ TRIzol 
(Thermo Fisher), after culture medium removal and PBS wash. RNA was purified over RNAeasy columns (Qiagen). 1 $\mu \mathrm{g}$ total RNA was used in a reverse-transcription (RT) reaction with SuperScript III and appropriate strand-specific reverse primer, according to manufacturer's instructions (ThermoFisher). $1 / 20^{\text {th }}$ of the RT reaction was used in a quantitative PCR reaction, using either 480 SYBR Green LightCycler PCR mix (Roche), SsoAdvanced Universal SYBR mix (Bio-Rad) or SYBR Green Master Mix (Applied Biosystems) and appropriate primers (see Extended Data Table 1), in triplicate reactions. RT-qPCR experiments were normalized against Gapdh or Rrm2 transcripts.

\section{Crosslinking and Immunoprecipitation of RNA and high throughput sequencing (iCLiP- Seq) for MATR3, PTBP1 and CELF1}

iCLIP experiments were performed as described in ${ }^{79}$. For CLiP-Seq all washes were conducted for 5 minutes per wash, at $4^{\circ} \mathrm{C}$ with ice cold buffers. Three confluent $15 \mathrm{~cm}$ plates of male tetOXist V6.5 (pSM33) ESCs ${ }^{24}$ were used per immunoprecipitation upon 6 hours of induction of Xist expression with $2 \mu \mathrm{g} / \mathrm{mL}$ doxycycline, and crosslinking was performed at $100 \mathrm{~mJ} / \mathrm{cm}^{2}$ at $4{ }^{\circ} \mathrm{C}$ in a Stratalinker 1800 (Stratagene). Crosslinked cells where harvested by scraping in cold 1xPBS and pelleted at $700 \mathrm{xg}$ for $2 \mathrm{~min}$. Cell pellets were lysed in ice cold lysis buffer [20 mM HEPES-KOH pH 7.5 (Sigma), 150 mM NaCl (Sigma), 0.6\% Triton X-100 (Sigma), 0.1\% SDS (Sigma), $1 \mathrm{mM}$ EDTA (GIbco), and $0.5 \mathrm{mM}$ DTT (Sigma)] and sonicated in a bioruptor (Diagenode) for 2x 15 minutes $(30 \mathrm{sec} \mathrm{ON}, 30 \mathrm{sec} \mathrm{OFF})$ on high setting at $4^{\circ} \mathrm{C}$. Sonicated lysates were cleared by centrifugation at $20,000 \mathrm{~g}, 5 \mathrm{~min}, 4 \mathrm{C}$, supernatants transferred to $15 \mathrm{~mL}$ falcon tubes and diluted in 5 volumes of buffer containing 20mM HEPES-KOH pH 7.5, $150 \mathrm{mM} \mathrm{NaCl}, 0.5 \mathrm{mM}$ DTT, 1.25x complete protease inhibitors EDTA-free (Roche), $50 \mu \mathrm{g} / \mathrm{ml}$ yeast tRNA (Life Technologies) and 400U RNAase out (Life Technologies). Samples were briefly mixed and rotated overnight at $4^{\circ} \mathrm{C}$. To prepare beads for pulldown, a magnet was used to isolate beads from $200 \mu \mathrm{L}$ of proteinGdynabead slurry, which were then washed $3 \mathrm{x}$ in $\mathrm{WB}_{150}[20 \mathrm{mM}$ HEPES-KOH pH 7.5, $150 \mathrm{mM}$ $\mathrm{NaCl}, 0.1 \%$ Triton-X100] and incubated overnight at $4^{\circ} \mathrm{C}$ with $50 \mu \mathrm{g}$ antibody $\alpha$-MATR3 (Abcam ab151714), $\alpha$-PTBP1 (Abcam, ab5642) in 700 $\mu \mathrm{L} \mathrm{WB}_{150}$. Beads were washed $3 \mathrm{x}$ in $\mathrm{WB}_{750}(20 \mathrm{mM}$ HEPES-KOH pH 7.5, 750mM NaCl, 0.1\% Triton-X100) and 1x with $\mathrm{WB}_{150}(20 \mathrm{mM}$ HEPES-KOH $\mathrm{pH} 7.5,150 \mathrm{mM} \mathrm{NaCl}, 0.1 \%$ Triton-X100) prior to incubation with lysate. After overnight 
incubation in lysate, beads were collected at the bottom of the falcon tube with a magnet and the supernatant was removed. Beads were then transferred to a $1.5 \mathrm{~mL}$ eppendorf tube with $1 \mathrm{~mL}$ of $\mathrm{WB}_{150}$, washed 5x in $\mathrm{WB}_{750}$ and 2x in PNK buffer (20 mM HEPES-KOH pH 7.5, $10 \mathrm{mM} \mathrm{MgCl2,}$ $0.2 \%$ Tween-20). The immunoprecipitated RNA was fragmented in $100 \mu$ of $1 \mathrm{x}$ MNase buffer (NEB) containing $5.0 \mu \mathrm{g}$ of yeast tRNA that was pre-warmed to $37^{\circ} \mathrm{C}$ in a thermomixer (Eppendorf) set to shake for $15 \mathrm{sec}$ ON/15 sec OFF at 750rpm (or minimum speed required to prevent settling of the beads). $50 \mu \mathrm{l}$ of $1 \mathrm{xMNase}$ buffer containing 60 gel units/ml (6 Kunz units/ml) of Micrococcal nuclease (NEB M0247S) were added and incubated for exactly for 5 min. The reaction was stopped with the reaction with $500 \mu$ of EGTA buffer (20 mM HEPES-KOH pH 7.5, $150 \mathrm{mM} \mathrm{NaCl}, 20 \mathrm{mM}$ EGTA, 0.1\% TritonX-100). The beads were then washed 4x in EGTA buffer and 2x in cold PNK buffer. The fragmented RNA was dephosphorylated in $100 \mu \mathrm{l}$ of $1 \mathrm{x}$ FastAP buffer (Fermentas) containing $0.15 \mathrm{U} / \mu \mathrm{l}$ of Fast alkaline phosphatase (Thermo Scientific, EF0651) and 0.2 U/ $\mu 1$ of RNaseOUT (LifeTechnologies, 10777-019), incubated in a thermomixer for $90 \mathrm{~min}$ at $37^{\circ} \mathrm{C}, 15 \mathrm{sec}$ shaking/20 sec rest. Beads were washed $4 \mathrm{x}$ in $\mathrm{WB}_{750}$ and $2 \mathrm{x}$ in cold PNK buffer. The dephosphorylated RNA was then ligated to a 3 'biotinylated linker RNA in $40 \mu \mathrm{L}$ of buffer containing $1 \mathrm{mM}$ ATP, 25\% PEG4000 (Sigma, 202398), $0.5 \mathrm{U} / \mu 1 \mathrm{~T} 4$ RNA ligase1 (NEB M0204S), 0.5 U/ $\mu$ l RNaseOUT, and 6.0 $\mu \mathrm{M}$ L3 linker (Extended Data Table 1). The ligation reaction was incubated in a thermomixer overnight at $16^{\circ} \mathrm{C}, 15 \mathrm{sec} \mathrm{ON} / 4 \mathrm{~min} \mathrm{OFF}$ at a speed that prevents beads from settling. The next day, beads were washed $4 \mathrm{x}$ in $\mathrm{WB}_{150}$ and $2 \mathrm{x}$ with cold PNK buffer. The RNA was then 5' end labeled in $24 \mu \mathrm{L}$ PNK wash buffer with $16 \mu \mathrm{L}$ of 1x PNK buffer (NEB) containing $150 \mu \mathrm{Ci}$ of gamma P32-ATP, $10 \mathrm{U}$ PNK and $1 \mathrm{U} / \mu \mathrm{L}$ of RNase OUT. The reaction was incubated in a thermomixer for 20 minutes at $37^{\circ} \mathrm{C}$ set to shake for $15 \mathrm{sec} \mathrm{ON} / 20 \mathrm{sec} \mathrm{OFF}$. The beads were then washed $3 \mathrm{x}$ with $\mathrm{WB}_{150}$. The immunoprecipitated complexes were eluted off the dynabeads in $50 \mu \mathrm{L}$ of buffer $(100 \mathrm{mM}$ Tris-HCl pH7.5, 0.6\% SDS, 5mM EDTA, 50mM DTT and $50 \mathrm{ng} / \mu \mathrm{L}$ yeast tRNA) incubated for $10 \mathrm{~min}$ at $85^{\circ} \mathrm{C}$ shaking continuously at $900 \mathrm{rpm}$. The elute was transferred to a new tube and the beads were rinsed with $1200 \mu \mathrm{L}$ of buffer $(50 \mathrm{mM}$ Tris- $\mathrm{HCl}$ $\mathrm{pH} 7.5,150 \mathrm{mM} \mathrm{NaCl}, 1.25 \mathrm{x}$ complete protease inhibitors (Roche), $50 \mathrm{ng} / \mu \mathrm{L}$ yeast tRNA and $0.1 \%$ Triton X-100) which was added to the first eluate. The combined eluates were centrifuged for $5 \mathrm{~min}$ at $4^{\circ} \mathrm{C}$ at maximum speed and the supernatant transferred to a new tube to prevent carry over of any remaining dynabeads. To prevent IgG heavy chain contamination that co-migrate with many 
proteins of interest, the biotinylated RNA-protein complexes were bound to monomeric avidin beads. To do this, $10 \mu \mathrm{L}$ packed monomeric avidin agarose beads (Thermo Fisher) were washed $3 \mathrm{x}$ with $\mathrm{WB}_{150}$. Beads were pelleted after each wash by spinning in a swing bucket rotor at $1000 \mathrm{xg}$, $4^{\circ} \mathrm{C}$ (use of the swing bucket rotor helps prevent loss of agarose beads). One packed bead volume was mixed with an equal volume of $\mathrm{WB}_{150}$ and $15 \mu \mathrm{L}$ of the bead slurry was added to each combined eluate and rotated at $4^{\circ} \mathrm{C}$ for 4 hours. The beads were then pelleted as above and washed $3 x$ with $\mathrm{WB}_{150}$. After the final wash, carefully remove the remaining $5-20 \mu \mathrm{L}$ of supernatant with a p10 pipette. The complexes were eluted off avidin beads by incubation in $30 \mu \mathrm{L}$ of buffer $(10 \mathrm{mM}$ Tris- $\mathrm{HCl} \mathrm{pH} 7.5,10 \%$ glycerol, $2.2 \%$ SDS, 5mM EDTA) at $85^{\circ} \mathrm{C}$ for 10 minutes in a thermomixer shaking at 900rpm. After centrifugation to pellet the beads, the supernatant was transferred to a new tube and mixed with $5 \mu \mathrm{L}$ of 1xLDS sample buffer (Life Technologies) with 300mM DTT. Samples were incubated at $90 \mathrm{C}$ for $10 \mathrm{~min}$ and then loaded on a pre-run $(75 \mathrm{v}, 10 \mathrm{~min}) \mathrm{NuPAGE}$ Bis-Tris Gel (Life Technologies NP0307) with 1x MOPS running buffer and run for 10-15min at $75 \mathrm{v}$ and then $120 \mathrm{~V}$ until each sample has been satisfactorily separated. The gel was then incubated in transfer buffer (25mM Bis-Tris, 25mM Bicine, 1mM EDTA pH7.2, 20\% methanol) for 5 minutes and then transferred onto a protran BA-85 nitrocellulose membrane using a semi-dry transfer apparatus (Biorad 170-3940) for 75min at 400mA (not exceeding 15V). After completion of the transfer, the membrane was briefly washed in milli-Q water, wrapped in plastic film and exposed on a phosophoimager screen for 1 hour. The regions of interest were then excised from the membrane and transferred to an eppendorf tube. The RNA was eluted from the membrane by incubation in $300 \mu \mathrm{L}$ of buffer $(100 \mathrm{mM}$ Tris-HCl pH $7.5,50 \mathrm{mM} \mathrm{NaCl}, 10 \mathrm{mM}$ EDTA and $2 \mu \mathrm{g} / \mu \mathrm{L}$ proteinase $\mathrm{K}$ ) for 30 minutes at $55^{\circ} \mathrm{C}$ in a thermomixer, shaking continuously. $300 \mu \mathrm{L}$ of prewarmed buffer $(100 \mathrm{mM}$ Tris-HCl $\mathrm{pH} 7.5,50 \mathrm{mM} \mathrm{NaCl}, 10 \mathrm{mM}$ EDTA, $7 \mathrm{M}$ urea and $2 \mu \mathrm{g} / \mu \Lambda$ proteinase K) was then added to the tube and incubated for a further $30 \mathrm{~min}$ at $55^{\circ} \mathrm{C}$. The supernatant was then transferred to a new tube and extracted with an equal volume of phenol:chloroform $(5: 1, \mathrm{pH} 4.5)$. The separated aqueous phase was precipitated with $0.5 \mu \mathrm{L}$ of Glycblue (Life Technologies), 60 $\mu \mathrm{L}$ Sodium Acetate pH 5.4 and $600 \mu \mathrm{L}$ isopropanol overnight at -20C. Next day, the RNA was pelleted by centrifugation at $4{ }^{\circ} \mathrm{C}$ for $30 \mathrm{~min}$ at max speed. The pellet was then washed with $1 \mathrm{~mL} 75 \% \mathrm{EtOH}$ before air drying for 2 minutes, and dissolved in $5.70 \mu \mathrm{L}$ RNAse free water and left on ice for 5-10 minutes before being reverse transcribed. To do this, 
$0.5 \mu \mathrm{L}$ of $10 \mathrm{mM}$ dNTPs and $0.5 \mu \mathrm{L}$ of $2 \mu \mathrm{M}$ RT primer (Extended Data Table 1 ) were added to the RNA, mixed by pipetting and denatured for $5 \mathrm{~min}$ at $70^{\circ} \mathrm{C}$ before being snap cooled on ice. The RT primers contain an 11nt Unique Molecule Identifier (UMI) used in sequence analysis (see below). The sample was then equilibrated at $25 \mathrm{C}$ in a PCR machine before $3.5 \mu \mathrm{L}$ of RT mix were added $(2 \mu \mathrm{L} 5 \mathrm{x}$ First Strand Buffer, $0.5 \mu \mathrm{L} 100 \mathrm{mM}$ DTT, $0.5 \mu \mathrm{L} 100 \mathrm{U} / \mu \mathrm{L}$ Superscript III (Life Technologies) and $0.5 \mu \mathrm{L} 40 \mathrm{U} / \mu \mathrm{L}$ RNase OUT (Life Technologies)) and incubated for 5 minutes at $25^{\circ} \mathrm{C}$ and then for 20 minutes at $42 \mathrm{C}$, and then 20 minutes at $48^{\circ} \mathrm{C}$. The reverse transcription reaction was then transferred to a new eppendorf tube containing $100 \mu \mathrm{L}$ TE, $11 \mu \mathrm{L} 3 \mathrm{M}$ Sodium Acetate and 2.5 volumes of $100 \% \mathrm{EtOH}$. The cDNA was precipitated overnight at $-20^{\circ} \mathrm{C}$, pelleted and washed as described above, dissolved in $5 \mu \mathrm{L}$ RNase-free water and then mixed with $7.5 \mu \mathrm{L}$ of formamide containing 10mM EDTA, bromophenol blue and xylene cyanol tracking dyes. For size determination, ladder was prepared as follows: $2 \mu \mathrm{L}$ GeneScan 500LIZ size marker (Life

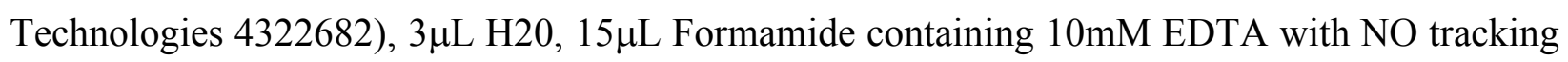
dyes. The samples and ladder were denatured for $5 \mathrm{~min}$ at $85^{\circ} \mathrm{C}$ and then loaded on a pre-run $5.5 \%$ (19:1 Bis:acrylamide) UREA-PAGE gel (1xTBE, 7.5M UREA) for $20 \mathrm{~min}$ at $21 \mathrm{~V}$. The gel was then scanned and a gel slice in the range of 70-120nt was excised, chopped into $1 \mathrm{~mm}$ cubes and the cDNA eluted in $700 \mu \mathrm{L}$ of TE buffer rotating at RT overnight. The next day, the cDNA was precipitated overnight as described above. The washed pellet was then dissolved in $6.7 \mu \mathrm{L}$ of RNase free water and left on ice for 5-10 minutes before being transferred to a PCR tube. Subsequently, the RNA was circularized by addition of $1.5 \mu \mathrm{l}$ of: $0.8 \mu \mathrm{L}$ of circligase II buffer, $0.4 \mu \mathrm{L} 50 \mathrm{mM} \mathrm{MnCl}_{2}$ and $0.3 \mu \mathrm{L}$ of $100 \mathrm{U} / \mu \mathrm{l}$ CicrLigase II ssDNA ligase (Epicentre CL9021K) and incubated in a PCR machine at $60^{\circ} \mathrm{C}$ for 60 minutes. The circularized cDNA was then digested with BamHI by addition of $30 \mu \mathrm{L}$ of: $4 \mu \mathrm{L} 10 \mathrm{x}$ FastDigest Buffer, $0.9 \mu \mathrm{L}$ of $10 \mu \mathrm{M}$ cut_oligo and $25.1 \mu \mathrm{L}$ RNase Free water. This mix was incubated for 4 minutes at $95 \mathrm{C}$ after which the temperature was decreased $1 \mathrm{C}$ each minute until $37^{\circ} \mathrm{C}$ after which $2 \mu \mathrm{L}$ of FastDigest BamH1 (Thermo Scientific FD0054) was added and incubated at $37^{\circ} \mathrm{C}$ for a further 30 minutes. The sample was transferred to an eppendorf tube and pelleted as described above. The pelleted DNA was dissolved in $12 \mu \mathrm{L}$ RNase free water. $2 \mu \mathrm{L}$ were used to prepare a $42 \mu \mathrm{L}$ PCR mix, containing $1 \mathrm{x}$ PFU buffer, 0.2mM dNTPs, $0.2 \mu \mathrm{M}$ P3 and P5 solexa primers, and 0.5U PFU polymerase. A negative control containing water instead of cDNA was also prepared. The $42 \mu \mathrm{L}$ reaction was then 
split into $4 \times 10 \mu \mathrm{L}$ reactions and PCR amplified for $20,24,28$ and 32 cycles $\left(94^{\circ} \mathrm{C} / 3^{\prime} ; 94^{\circ} \mathrm{C} / 30 \mathrm{~s}\right.$; $63.5^{\circ} \mathrm{C} / 15 \mathrm{~s} ; 72^{\circ} \mathrm{C} / 30 \mathrm{~s}$; with final extension at $72^{\circ} \mathrm{C}$ for 7 minutes). The PCR amplicons were run on a $2 \%$ agarose gel in $0.5 \mathrm{X} \mathrm{TBE} / \mathrm{EtBr}$ and the number of cycles required to produce $50-200 \mathrm{ng}$ of PCR product from the remaining $10 \mu \mathrm{L}$ of cDNA template was calculated. The PCR reaction was repeated using $10 \mu \mathrm{L}$ remaining ssDNA template, run on a $2 \%$ gel as before and the $150-210 \mathrm{bp}$ size range was excised and purified using Zymoclean Gel DNA recovery kit (Zymo Research D4007). DNA concentration was determined by qubit using the dsDNA Broad Range assay and prepared for sequencing on an Illumina HiSeq2000 machine using a single end 100bp protocol.

\section{Enhanced Crosslinking and Immunoprecipitation of RNA and high throughput sequencing (eCLIP) for CELF1}

eCLIP experiments against CELF1 ( $\alpha$-CELF1 (ab129115) were performed as described in ${ }^{80}$ with a few modifications. As with iCLIP, male tet-inducible -Xist V6.5 ESCs (pSM33) were induced with $2 \mu \mathrm{g} / \mathrm{mL}$ doxycycline for 6 hours prior to crosslinking at $100 \mathrm{~mJ} / \mathrm{cm}^{2}$ at $4{ }^{\circ} \mathrm{C}$ in a Stratalinker 1800 (Stratagene). Cells were then processed according to the eCLIP protocol for input and immunoprecipitated samples until cDNA was obtained. We then used the iCLIP protocol from the gel-purification of the cDNA through to amplification and purification of the DNA library. For eCLIP samples were sequenced on an Illumina HiSeq2000 machine using the single end 50bp protocol.

\section{CLIP-seq analysis}

CLIP-seq results were mapped using TopHat and processed with the publicly available fastq-tools, fastx-toolkit, Samtools, Bedtools, DeepTools and UCSC scripts ${ }^{8182}$. The first 11 bases of each sequenced read correspond to a UMI, composed of a library specific barcode ( $3 \mathrm{nt}$ ) flanked by 4 degenerate nucleotides. The UMI permitted removal of PCR duplicates from the total sequenced reads with the fastq-uniq command-line tool. The Fastx-toolkit was then used to clip 3' adapter sequences. Sequences shorter than $20 \mathrm{nt}$ were discarded. Reads were then de-multiplexed and mapped to the iGenome mm9 reference genome by Tophat with high stringency settings (no mismatches, reads with multiple genome hits were discarded). Library-depth normalized counts 
were generated and data was converted to bigWig format to visualize tracks in IGV. Peaks were called using CLIPper ${ }^{83}$ using the --superlocal option.

\section{ChIP-seq}

$3 \times 15 \mathrm{~cm}$ plates of male tetOXist- $\Delta$ Tsix V6.5 ESCs ( 100 million cells) were used to prepare chromatin for PTBP1 ChiP-seq. Cells were induced for 0 or 20 hours with $2 \mu \mathrm{g} / \mathrm{mL}$ doxycycline to induce Xist prior to harvesting by trypsinization. Cells were then pelleted by centrifugation at $700 \mathrm{~g}$ for 5 minutes at RT and resuspended in a total volume of $10 \mathrm{~mL}$ PBS. The wash step was repeated twice before resuspending in $10 \mathrm{mLs}$ of $1 \mathrm{x}$ PBS and transferring to a $50 \mathrm{~mL}$ falcon tube to which Disuccinimidyl glutarate (DSG) (Pierce) in DMSO was added for crosslinking to a final concentration of $2 \mathrm{mM}$ and incubated for 10 minutes at room temperature with gentle mixing under standard laboratory safety practices. Cells were then pelleted and the supernatant was safely disposed of. Cells were re-suspended in 10mL ESC medium and incubated for 10 minutes with $1 \%$ formaldehyde (16\% methanol free, Pierce) at room temperature with gentle mixing under standard laboratory safety practices. The reaction was quenched by addition of freshly made 0.125M Glycine (Sigma) for 5 minutes at room temperature. Cells were pelleted and supernatant was safely disposed of. Cells were washed twice in 50mL PBS with protease inhibitors (Complete EDTA free, Roche) before being pelleted and flash frozen in liquid nitrogen and stored at -80C. The frozen pellets were processed for ChIP-seq as described in 69

\section{ChIP-seq analysis}

Reads were mapped using Bowtie to the iGenome mm9 reference genome. Duplicate reads were removed and length extended to 49 nt. Normalized reads count were generated across 50nt bins. Tracks were visualized in IGV in bigWig format.

\section{RNA Affinity Purification (RAP)}


RAP was performed as described $\mathrm{in}^{24}$. For the RAP-seq experiment, we used male T20 ESCs ${ }^{6}$ carrying homing site in the Hprt locus on the single X-chromosome as well as a tetracyclineinducible transactivator in the R26 locus. The Hprt homing site includes a bidirectional, tetracycline-inducible promoter for expression of a control gene $(E G F P)$ and of the Xist cDNA transgene introduced later by site-specific recombination, as well as a lox $\mathrm{P}$ site neighboring the tet-promoter and linked to a truncated neomycin-resistance gene lacking a promoter and translation initiation codon. We integrated two different Xist cDNA transgenes into the homing site by electroporation of the respective Xist cDNA encoding plasmid and a Cre expression plasmid. The Xist transgene plasmid contained a promoter-less Xist sequence followed by a poly-adenylation signal and a PGK promoter and translation initiation codon linked to a loxP site. Site-specific recombination of the lox $\mathrm{P}$ sites in the Xist plasmid and the homing site linked the translation initiation codon and Pgkl promoter to the neo gene, which restored the antibiotic resistance marker. A single copy of the Xist cDNA transgene was thus integrated under the control of the inducible promoter. In this study, we employed a $\sim 14.5 \mathrm{~kb}$ Xist cDNA with either a 4122 nucleotide deletion between BstEii sites within the Xist cDNA, deleting the E-repeat and surrounding sequences, or a 1237 nucleotide deletion ending at a similar region with Xist and not including the E-repeat, which was generating by deleting internal sequences within the cDNA by SnaBI digestion and re-ligations (see Fig. $2 \mathrm{~g}$ ). Cells were induced with $2 \mu \mathrm{g} / \mathrm{mL}$ dox for $6 \mathrm{~h}$, prior to fixation for RAP-seq.

\section{Microscopy}

Epifluorescence imaging:_Immunofluoresence and RNA FISH samples were imaged using a Zeiss AxioImager M1 microscope with a 63x objective and acquired with AxioVision software. Epifluorescence images shown are sections and were analyzed, merged and quantified using ImageJ or Adobe Photoshop.

Xist aggregation analysis: To quantify the aggregation of Xist clouds, images were taken as Zstacks and transformed in a Maximum Intensity Projection (MIP) image to detect the entire Xist FISH signal in one plane. The background was removed using a rolling ball radius of 50 pixels. Xist RNA cloud areas were measured by creating a binary mask over the Xist RNA FISH signal 
for each analyzed Xist cloud. Edges of each Xist cloud signal were determined by selecting a central pixel and all associated pixels of same intensity value (+/- 5 units). The ImageJ FracLac plugin was then used to calculate the area of a circle encompassing each cloud signal ${ }^{84}$. The ratio of the Xist cloud area over its bounding circle area approximates the compaction of the Xist RNA cloud. Significant differences between $\mathrm{WT} / \triangle \mathrm{E}$ ESC or siRNA treated samples were tested with the non-parametric 2 sample Kolmogorov-Smirnov (K-S) test.

3D-Structured Illumination Microscopy (3D-SIM): 3D-SIM super-resolution imaging was performed on a DeltaVision OMX V3 system (Applied Precision, GE Healthcare) equipped with a $100 \AA \AA \sim 1.40$ NA Plan Apo oil immersion objective (Olympus, Tokyo, Japan), Cascade II:512 EMCCD cameras (Photometrics, Tucson, AZ, USA) and 405, 488 and $593 \mathrm{~nm}$ diode lasers. Image stacks were acquired with a z-distance of $125 \mathrm{~nm}$ and with $15 \mathrm{raw}$ images per plane (five phases, three angles). The raw data were computationally reconstructed with the soft-WoRx 6.0 software package (Applied Precision) using a wiener filter set at 0.002 and channel-specifically measured optical transfer functions (OTFs) using immersion oil with different refractive indices (RIs) as described in ${ }^{85}$. Images from the different channels were registered using alignment parameters obtained from calibration measurements with $0.2 \mu \mathrm{m}$ diameter TetraSpeck beads (Invitrogen) as described in ${ }^{75}$.

Improved Confocal microscopy: Improved confocal laser scanning microscopy was performed on a LSM880 platform equipped with 100x/1.46NA or 63x/1.4 NA plan Apochromat oil objectives and 405/488 diode and 594 Helium-Neon lasers using the Airyscan detector (Carl Zeiss Microscopy, Thornwood, NY). An appropriate magnification was used in order to collect image stacks from a region that encompassed the nucleus of interest thereby optimizing imaging time and reducing photobleaching. The pixel size and z-optical sectioning were set to meet the Nyquist sampling criterion in each case. Airyscan raw data were linearly reconstructed using the ZEN 2.3 software.

\section{Quantitative image analysis}


All image analysis steps were performed on Fiji/Image ${ }^{86,87}$.

CELF-1 intensity plot profiles: Airyscan image stacks were imported into ImageJ and converted to 16-bit composites. The 3D-stacks were reduced into $2 \mathrm{D}$ images and $2 \mu \mathrm{m}$ intensity line plots were used to extract the intensity profiles over the Xi enriched signal in the CELF1 channel. The same line-plot was used in a random nucleoplasmic region to select for the average nuclear CELF-1 intensities. The ratio of the top $10 \%$ intensities of the signals were plotted after diving over the nucleoplasmic signal.

Xi DAPI intensities quantification: Wide-field image stacks were generated from 3D-SIM raw data of H3K27me3 and DAPI stained cells by average projection of five consecutive phase-shifted images from each plane of the first angle and subjected to an iterative 3D deconvolution using soft-WoRx 6.0 software. The reconstructed image stacks were imported to ImageJ and converted to 8-bit files. In order to measure the $\mathrm{Xi}$ underlying DAPI intensity, binary masks from the H3K27me3 channel were created to define the Xi territory of day 7 WT and $\triangle \mathrm{E}$ ESC nuclei. A threshold was carefully applied selecting the boarder of H3K27me3 enriched region that demarcates the Xi territory. Subsequently the grey values of the corresponding masked region in the DAPI channel were extracted and plotted.

Segmentation of Xist RNA foci from 3D-SIM datasets: The 32-bit reconstructed 3D-SIM image stacks were imported into ImageJ were grey values were shifted to the positive range and converted to 16-bit composites after subtracting the mode grey value to remove background noise. Segmentation of Xist RNA foci was performed by using the TANGO plugin ${ }^{88}$ on ImageJ according to the pipelines described in ${ }^{75}$. In brief, nuclear masks were created by using the nucleus processing chain. Xist foci were segments by first pre-filtering with a TopHat filter with a radius of 1 pixel in all three dimensions (xyz), followed by a Laplace of Gaussian filter with a radius of 1-pixel $(\mathrm{x}, \mathrm{y}, \mathrm{z})$. Segmentation of foci was performed using the spot detector 3D with Otsu autothresholding. Segmented objects were post-filtered with a size and edge filter of 5 pixels per spot and an SNR above 2. 
Amira reconstructions: 3D-reconstructions were performed using Amira 2.3 (Mercury Computer Systems, Chelmsford, Massachusetts, United States). Image stacks were imported into Amira as separate channels. Xist FISH or antibody stainings were reconstructed as surface renderings while DAPI was reconstructed as volume rendering using the Volren module that allows visualization of intensity in color maps.

\section{Genomics data}

Genomics data (CLIP-seq, ChIP-seq and RAP-seq) will be made accessible with publication on the GEO database.

Technical Note 1: The Xist and MS2 DNA FISH probes were synthesized using Klenow Exopolymerase primed with random hexamers from DNA templates. Specifically, for the Xist probe, we used a plasmid encoding the entire $17.9 \mathrm{~kb}$ Xist cDNA as template. The MS2 probe was synthesized from a PCR product that amplified the $1.1 \mathrm{~kb}$ MS2 repeat sequence from genomic DNA of targeted F1 2-1 ESCs. See Fig. 2a for illustration of the sequences covered by the two FISH probes. The difference in sequence length available for the probes to bind to Xist transcripts in cells meant that the Xist probe gave more intensely fluorescent FISH signals than the MS2 probe. To assign the cas or 129 allele-of-origin for the Xist cloud, we first looked for Xist probe signal then asked whether we could detect any co-localizing MS2 probe signal. Thus, while we show that $50-70 \%$ of the cells with a detectable Xist cloud at late stages of initiation (day 4 onward) still show MS2 co-localizing signal cloud formation in $\Delta \mathrm{E}$ cells, we wish to emphasize that almost none of these clouds presented as morphologically intact and our results, as presented, underrepresent the penetrance of the dispersal phenotype observe for $\Delta \mathrm{E}$-Xist transcript at later stages of XCI initiation. 
a

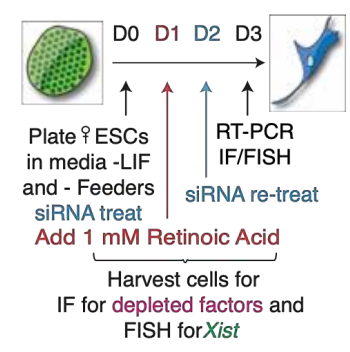

c $\mathrm{i}$

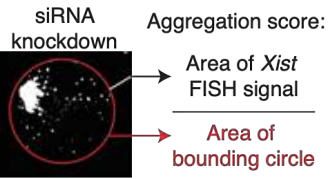

C ii

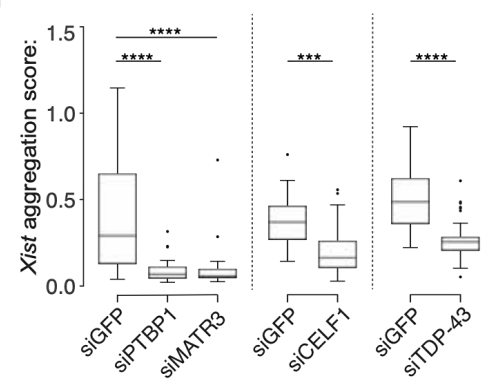

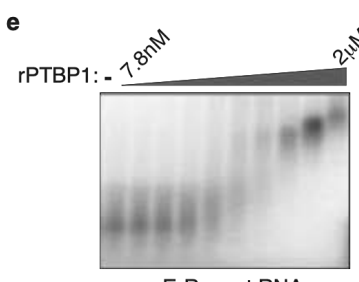

E-Repeat RNA

g

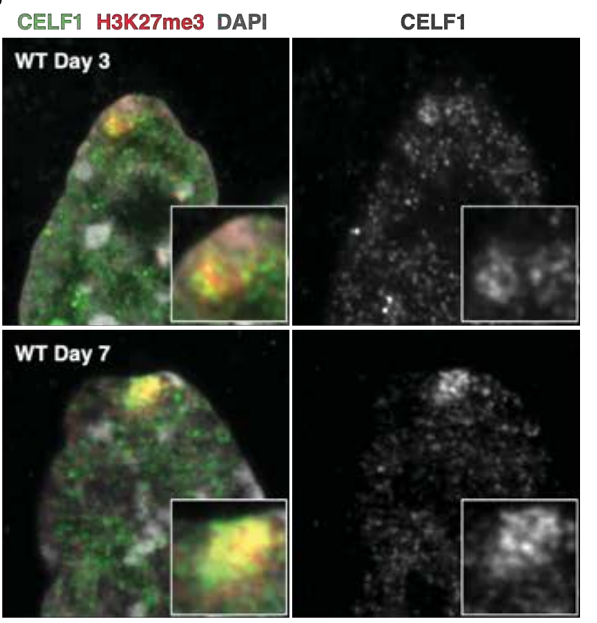

b
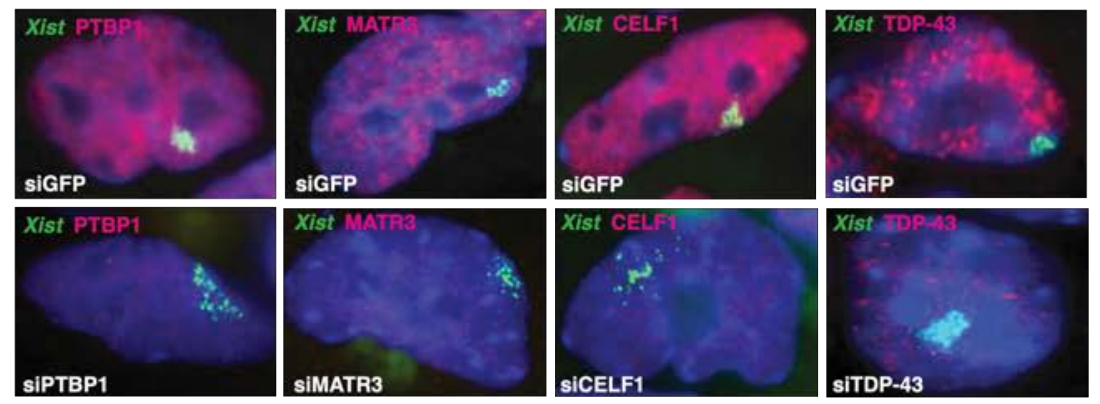

d $\mathbf{i}$
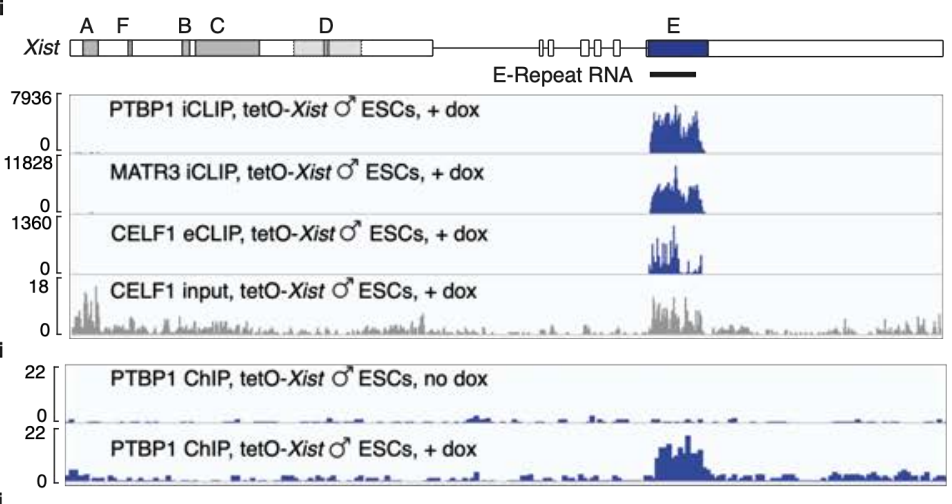

iii

132 [ PTBP1 iCLIP (transgene), $ๆ$ P1 brain

$116[$ PTBP2 ICLIP, $\uparrow$ P1 brain 298 TDP-43 iCLIP + E18 brain

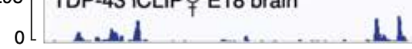

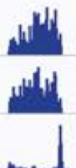

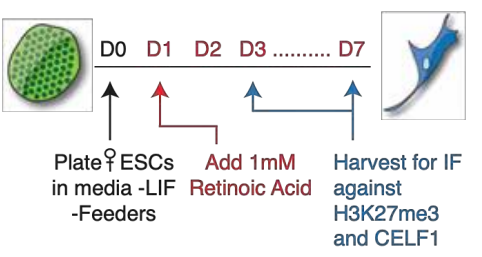

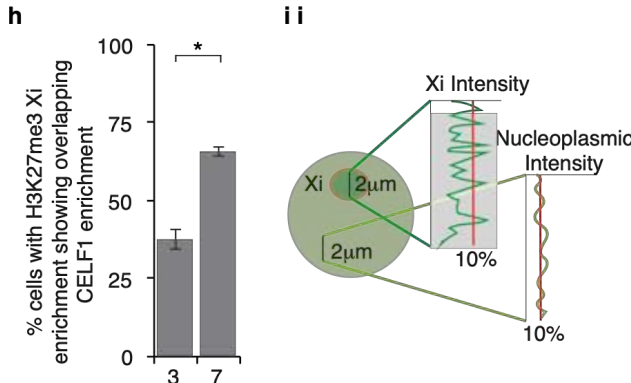

Day of differentiation i ii

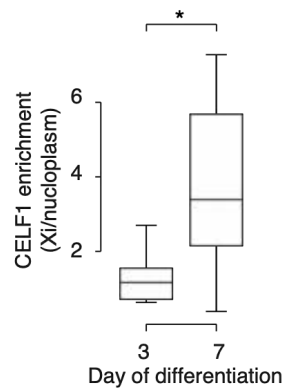




\section{Figure 1: PTBP1, MATR3, TDP-43 and CELF1 bind the Xist E-repeat}

a, Protein knockdown approach in differentiating female ESCs.

b, Representative images of siRNA-treated differentiating female ESCs immunostained for indicated proteins (red) and probed for Xist by RNA FISH (green).

c i, Depiction of aggregation score calculation. Maximum Intensity Projections (MIPs) of stacked images were used to mask the Xist RNA FISH signal and calculate the smallest bounding circle that encompassed the entirety of the Xist mask. The aggregation score is the ratio of the Xist mask (in pixels) to the bounding circle (in pixels). ii, Box plots showing Xist aggregation scores upon depletion of indicated proteins. Depletion experiments for CELF1 and TDP-43 were conducted separately to PTBP1 and MATR3, and measured relative to their paired siGFP (control) sample. Significance testing was performed using a 2 -sample K-S test $(* * * \mathrm{p}<0.0005, * * * * \mathrm{p}<0.00005)$. No significant differences $(\mathrm{p}>0.05)$ were observed between different siGFP treated samples. 2536 clouds were measured per sample from one experiment.

d i, Top: Diagram of the Xist locus with introns, exons and repeat arrays A-F of the RNA depicted. The repeat regions (A-D and F) within exon 1 are shown in grey. The sole conserved D-repeat in mouse (dark grey) is flanked by truncated versions (light grey coloring/dotted line). The $1.4 \mathrm{~kb}$ Erepeat is shown in blue. The in vitro transcribed E-repeat RNA used in (e) is indicated. PTBP1 (iCLIP), MATR3 (iCLIP) and CELF1 (eCLIP) CLIP-seq profiles across the Xist locus after 6 hours of doxycycline (dox) treatment in male tetO-Xist ESCs are shown, together with the CELF1 input sample. ii, PTBP1 ChIP-seq profiles across the Xist locus before or after 20 hours of dox treatment in male tetO-Xist ESCs. iii, PTBP1, PTBP2 and TDP-43 iCLIP-seq profiles across the Xist locus in female mouse brain.

e, Left: Radiograph of an EMSA with in vitro transcribed E-repeat RNA (see (d)) and either none, or increasing amounts of recombinant (r) PTBP1 (0, 7.8nM, 15.6nM, 31.3nM, 62.5nM, 125nM, 250nM, 500nM, $1 \mu \mathrm{M}$ and $2 \mu \mathrm{M})$. Right: Quantification showing fraction of bound RNA. Quantification from two independent experiments, with standard error of the mean (SEM) shown. f, Depiction of wildtype female ESC differentiation experiment for immunostaining.

g, Left: Confocal-airyscan optical sections through nuclei of WT female cells at day 3 or 7 of differentiation, immunostained for CELF1 (green) and H3K27me3 (marking the Xi, red) and counterstained with DAPI (gray). Inset: Enlargement of the Xi-territory. Right: Same as left only 
showing CELF1 staining in greyscale. Note the increased CELF1 staining intensity within the H3K27me3-enriched territory with differentiation time.

h, Histogram showing the number of H3K27me3-marked Xi's with a co-localizing CELF1 enrichment on days 3 and 7 of differentiation. 50-90 cells with H3K27me3 enrichment were counted per coverslip. 3 coverslips taken from 2 independent differentiations were counted for each sample. Error bars indicate SEM (p-value: $\mathrm{p}<0.05$, 2-tailed students t-test).

i i, Intensity values for CELF1 fluorescence were recorded across a $2 \mu \mathrm{m}$ line plot over the Xi (as marked by H3K27me3 staining) or in the nucleoplasm of the same nucleus in z-stack projections, as illustrated in the schematic. ii, Box plot showing the distribution of the ratio between the top $10 \%$ CELF1 intensity values within the $\mathrm{Xi}$ compared to the top $10 \%$ intensity values within the nucleoplasm for 12 cells per time point from one experiment. 
a

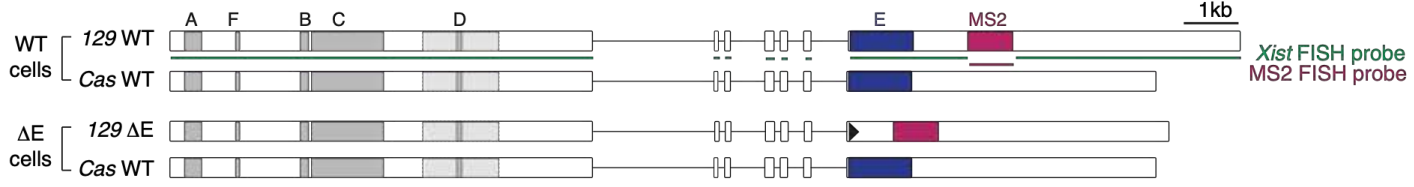

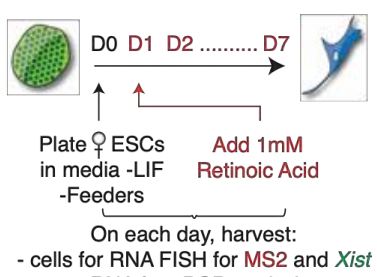
RNA for qPCR analysis
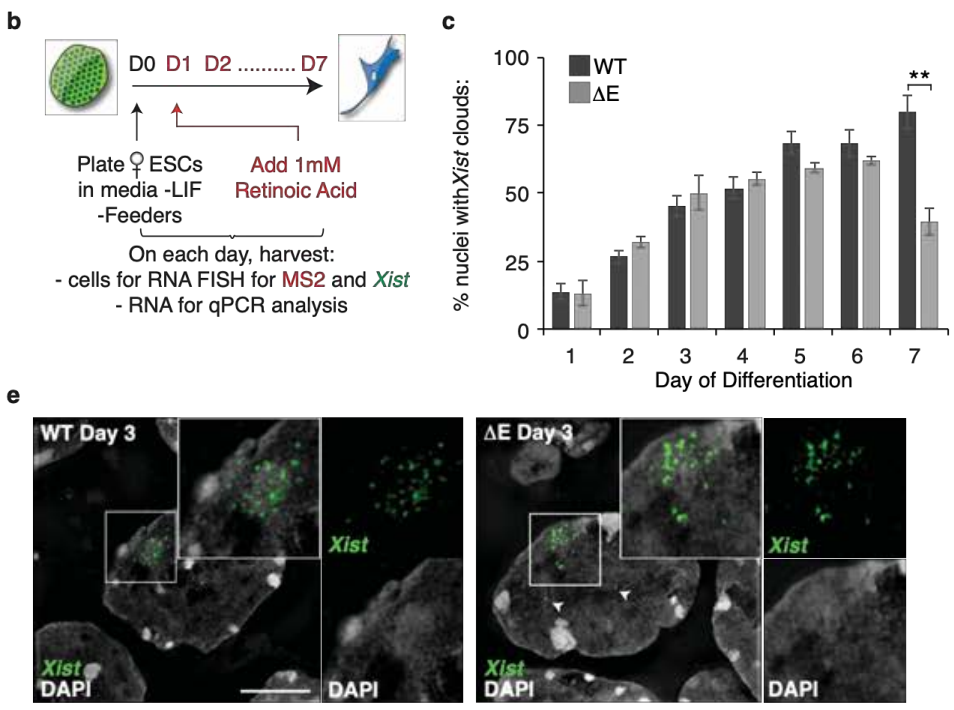

g
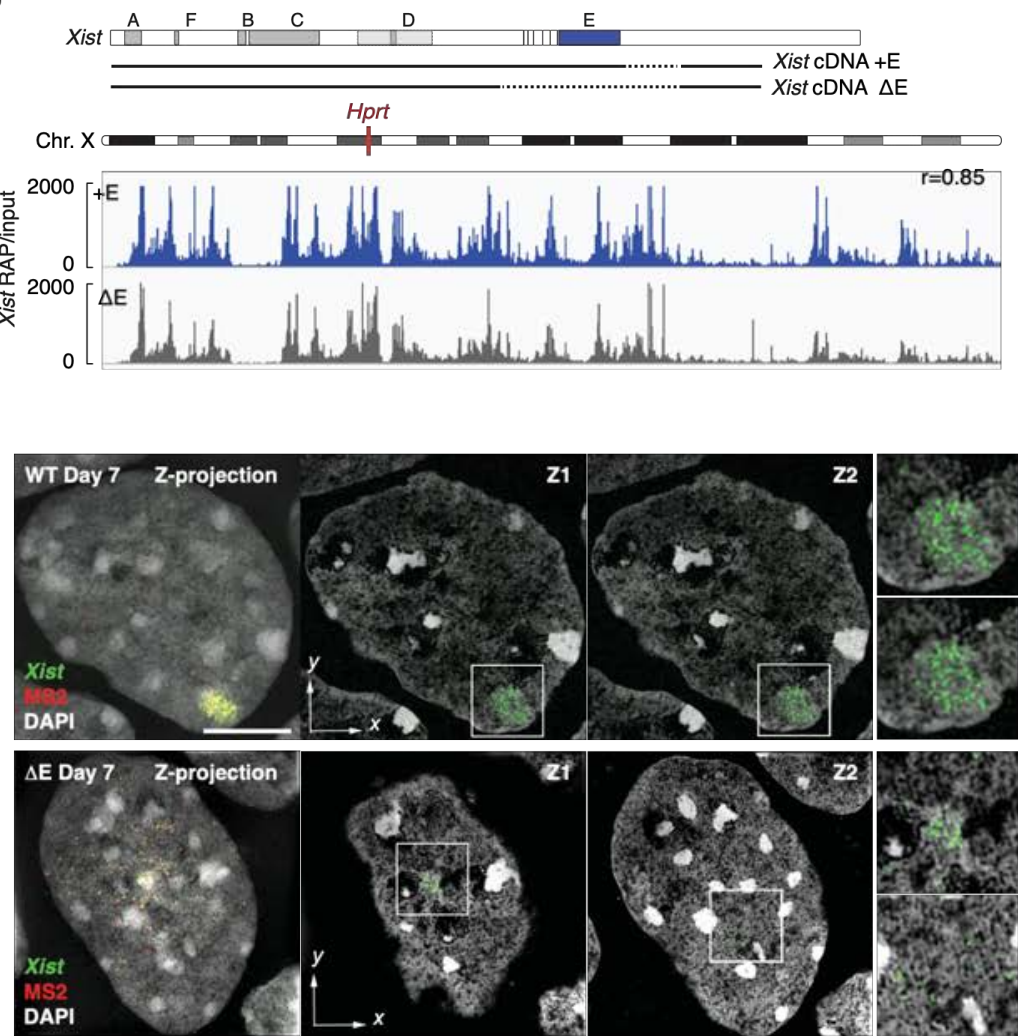
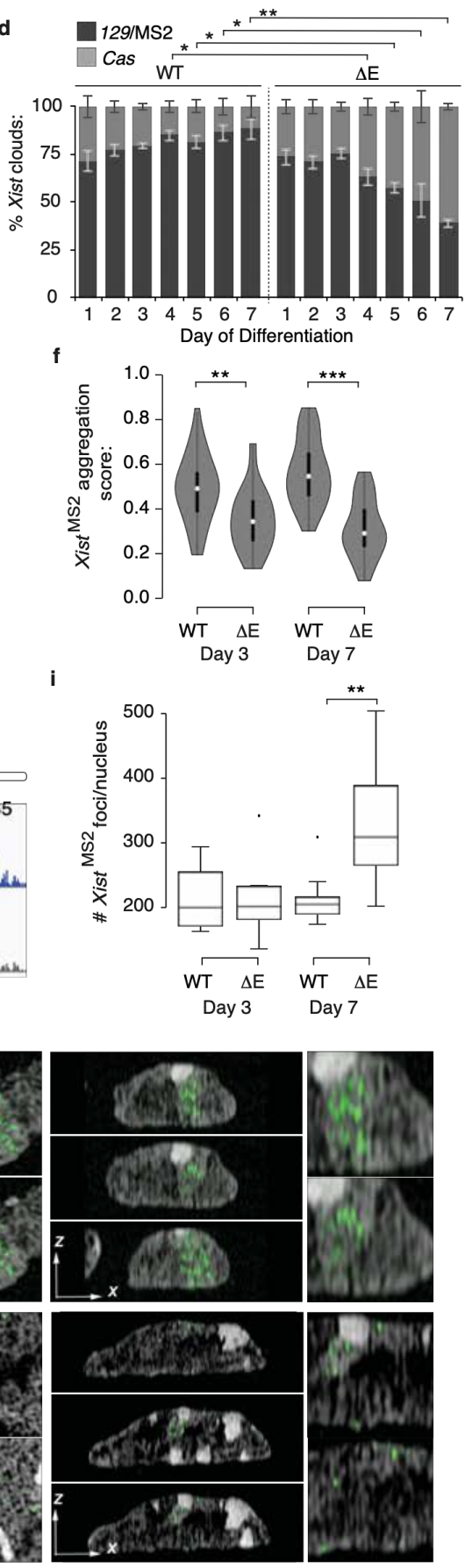


\section{Figure 2: The E-repeat is required for Xist localization and controls the number of Xist foci} a, Diagram showing Xist alleles in female WT and $\triangle \mathrm{E}$ ESCs. A $11 \mathrm{xMS} 2$ hairpin, located 1.2kb downstream of the $1.4 \mathrm{~kb}$ E-repeat, is indicated in magenta. Green lines delineate the sequences within Xist RNA targeted by the Xist FISH probe. The magenta line represents the region targeted by the MS2 FISH probe. Note that the loxP site (black triangle) present in the genomic sequence after targeting is spliced out in $\Delta \mathrm{E}$ Xist transcripts (see Extended Data Fig. 6).

b, Scheme of Retinoic Acid (RA) differentiation experiment to assess Xist coating of the Xi in female WT and $\triangle \mathrm{E}$ ESCs.

c, Histogram showing the number of nuclei that display an Xist cloud, detected by RNA FISH against Xist, at the indicated day of differentiation. $100-250$ cells were counted per time point across 3 independent differentiations. Error bars represent SEM (p-values: ** $<0.005$, 2-tailed students t-test).

d, Histogram showing the allelic origin of Xist RNA clouds for the data in (c), detected by RNA FISH for Xist and the MS2 tag. At later days of differentiation (4-7), MS2+Xist ${ }^{129}$ (mutant) clouds in $\Delta \mathrm{E}$ cells were scored if an Xist signal (which delivers a brighter signal than the MS2 probe due to target sequence length - see (a)) co-localized with even a weak MS2 signal. 50-80 Xist clouds were assessed per time point, across 3 independent experiments. Error bars represent SEM (pvalues: $*<0.05, * *<0.005,2$-tailed students t-test).

e, 3D-SIM optical sections through WT and $\Delta \mathrm{E}$ cells at day 3 of differentiation showing Xist RNA FISH signals (green) of MS2+Xist ${ }^{129} \mathrm{WT}$ or $\Delta \mathrm{E}$ clouds, with DAPI counterstaining in grey. Note, that for simplicity, the MS2 signal is not shown, but the Xist clouds shown are derived from the MS2+Xist ${ }^{129}$ allele. Inset: Enlargement of marked region corresponding to the Xist-coatedchromosome. Right: Same as inset except DAPI and Xist RNA FISH are presented independently, highlighting formation of the Barr body. Bar; $5 \mu \mathrm{m}$. Arrowheads indicate $\Delta \mathrm{E}-X i s t^{\mathrm{MS} 2}$ foci located further from the core of the Xist cloud.

f, Violin plots of aggregation scores of MS2+Xist ${ }^{129}$ clouds formed in WT and $\Delta \mathrm{E}$ cells at days 3 and 7 of differentiation. Only MS2+Xist ${ }^{129}$ clouds were chosen for the analysis, but the signal quantified was derived from the co-localizing Xist RNA FISH signal. Significance testing was performed using a 2-sample K-S test. 30-34 clouds were analyzed per time point from one experiment (p-values: $* *<0.005, * * *<0.0005)$. 
g, Top: Diagram of the tet-inducible Xist cDNA transgenes that were inserted into the Hprt locus on the X-chromosome in male ESCs for RAP-seq. Bottom: RAP-seq profile of $+\mathrm{E}$ and $\Delta \mathrm{E} X i s t$ across the X-chromosome after 6 hours of dox treatment. Pearson correlation score between the two profiles is indicated. Data were collected from one experiment.

$\mathbf{h}$, Left panel: 3D-SIM MIP of the Xist RNA FISH signal from MS2+Xist ${ }^{129}$ clouds in WT (top panel) and $\Delta \mathrm{E}$ (bottom panel) nuclei at day 7 of differentiation. Xist is shown in green, MS2 in red, DAPI in grey. Bar; $5 \mu \mathrm{m}$. Next two large panels: Xist/DAPI signals from two different Z-plane optical sections through the nucleus are shown. Next two small panels: Enlargements of the Xistcoated X-chromosome from each Z-plane (Z1, top; Z2, bottom). Rightmost panels: Y-plane sections through same cells, showing Xist localization relative to the apical and basal nuclear lamina with enlargements of area with Xist signal area shown on the far right (see Extended Data Figure 8a for additional images).

i, Box plot showing the distribution of Xist RNA foci number derived from the MS2+Xist ${ }^{129}$-allele in WT and $\Delta \mathrm{E}$ cells at day 3 and 7 of differentiation. Significance testing was performed using the 2-sample K-S test. 10 clouds were quantified per condition from one experiment (p-value: **< $0.005)$. 
bioRxiv preprint doi: https://doi.org/10.1101/2020.03.09.979369; this version posted March 12, 2020. The copyright holder for this preprint (which was not certified by peer review) is the author/funder. All rights reserved. No reuse allowed without permission.

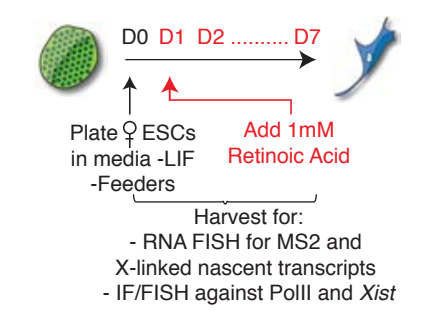

b
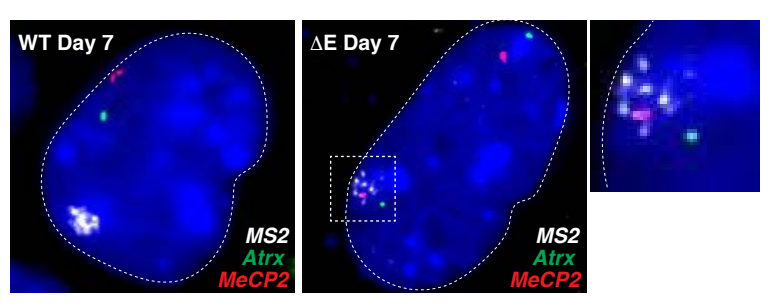

$\left.\begin{array}{l}\text { Single spot } \\ \text { co-localizing with Xist } \\ \text { Bi-allelic }\end{array}\right] \begin{aligned} & \text { Lack of } \\ & \text { silencing }\end{aligned}$

Rnf12

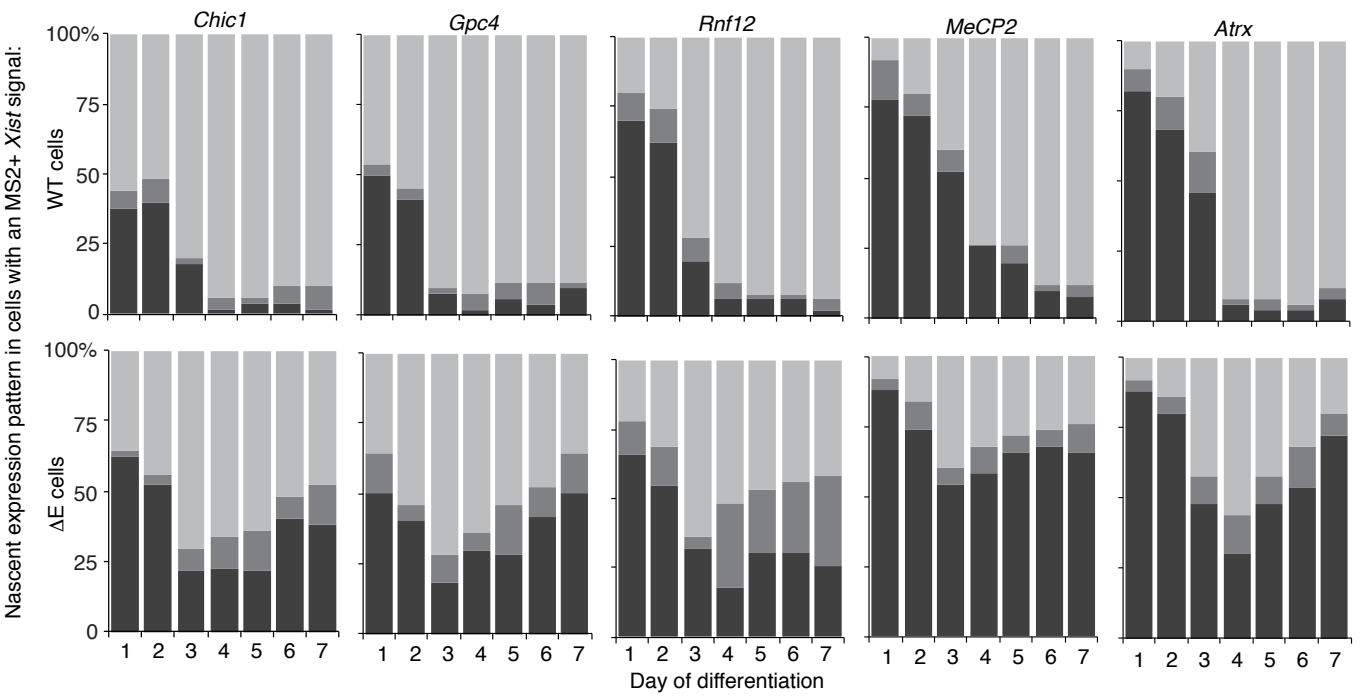

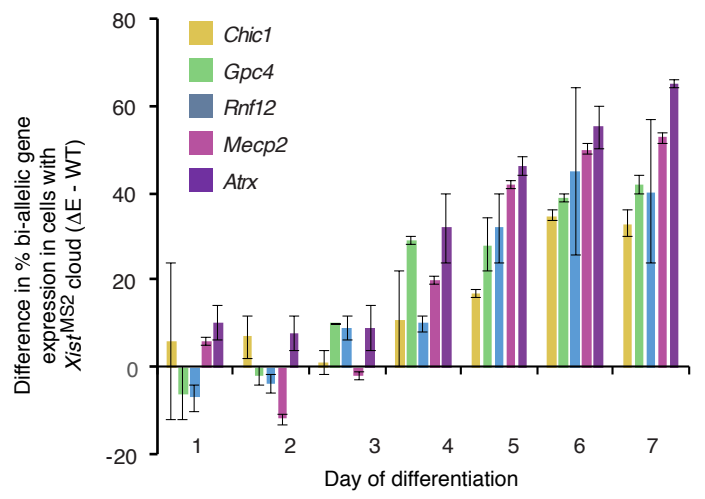

e
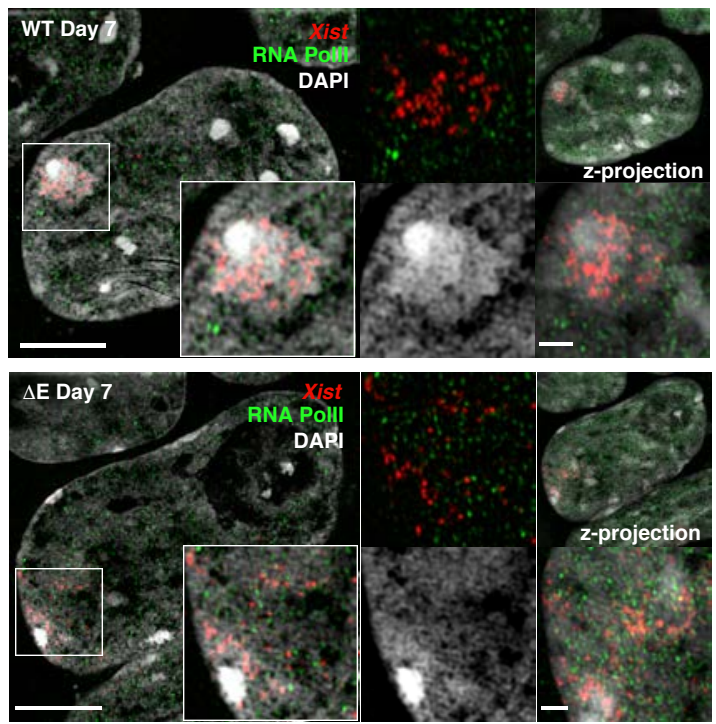


\section{Figure 3: The Xist E-repeat establishes a memory of gene silencing during XCI initiation}

a, Scheme of RA-differentiation experiment assessing gene silencing and Xi RNA-PolII presence in female WT and $\triangle \mathrm{E}$ ESCs.

b, Representative epifluorescence images showing the nascent expression patterns of the X-linked genes Mecp2 (red) and Atrx (green) found in a majority of WT (mono-allelic expression on the active $\mathrm{X}$ chromosome) and $\Delta \mathrm{E}$ cells (bi-allelic expression) with $\mathrm{MS} 2+X i s t^{129}$ signal at day 7 of differentiation, together with the MS2 RNA FISH signal (white). DAPI staining is shown in blue. Note that MS2 signal in $\Delta \mathrm{E}$ cells is faint as it is dispersed across the nucleus as described in Fig. 2. Far right: enlargement of square hatched region showing the MS2 FISH signal in $\Delta \mathrm{E}-X i s t$ cells. c, Histograms showing the quantification of nascent expression patterns of indicated X-linked genes in WT and $\Delta \mathrm{E}$ cells displaying an MS2+Xist ${ }^{129}$-coated X-chromosome, across 7 days of differentiation. 50 cells with an MS2+Xist ${ }^{129}$ clouds were counted per time point. (See Extended Data Fig. 12c/d).

d, Histogram showing the percentage difference in bi-allelic nascent gene expression (lack of silencing) between $\Delta \mathrm{E}$ and WT cells with an MS2+Xist ${ }^{129}$ cloud, across 7 days of differentiation for the X-linked gene expression quantification shown in (c and Extended Data Fig.12d). Error bars represent SEM.

e, 3D-SIM optical sections through nuclei of WT and $\Delta \mathrm{E}$ cells showing Xist clouds derived from the MS2+Xist ${ }^{129}$ allele at 7 days of differentiation, stained for RNA-polII (green) and probed for Xist (red). DAPI is shown in grey. Inset: Magnification of the marked region. Right, top: Same as inset but only showing PolII and Xist signals. Right bottom: Same as inset but only showing DAPI. Far right: z-stack projections of the whole nucleus (top panel) and Barr Body (lower panel). Size bars: $5 \mu \mathrm{m}$; inset: $1 \mu \mathrm{m}$. 

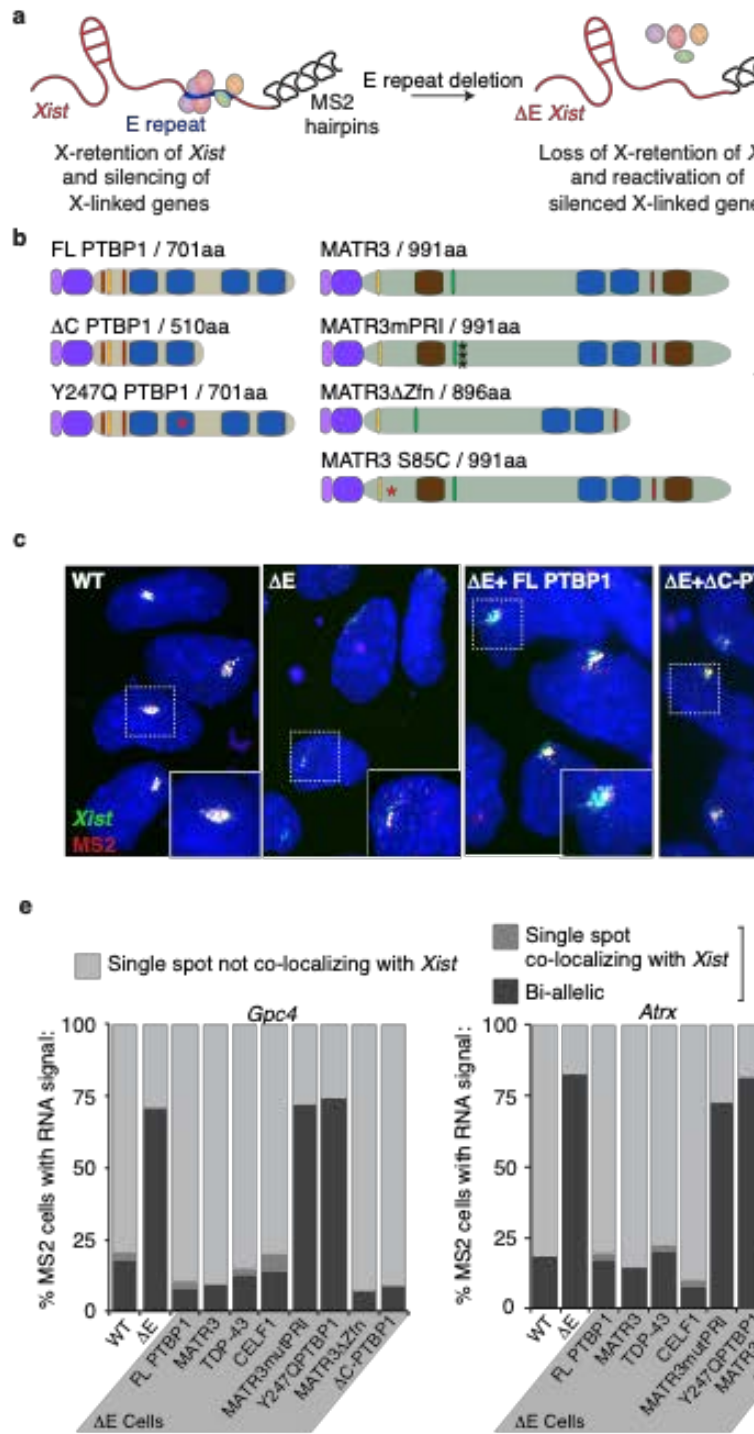
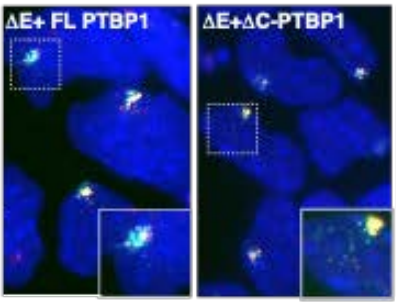

Single spot

\begin{tabular}{l|l} 
Single spot & Lack of \\
colizing with Xist & $\begin{array}{l}\text { Lack } \\
\text { silencing }\end{array}$
\end{tabular}

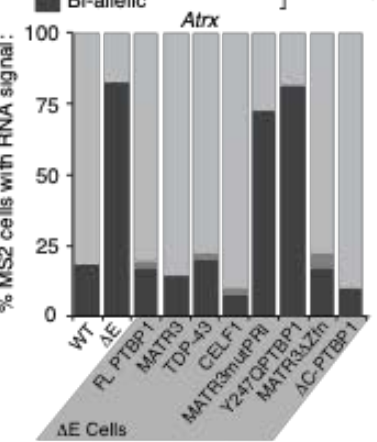

TDP-43 / 558aa COMOI

TDP-43 4xM / 558aa (S48E*, W334G*, W385G", W412G")

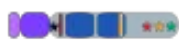
CELF1 / 630aa

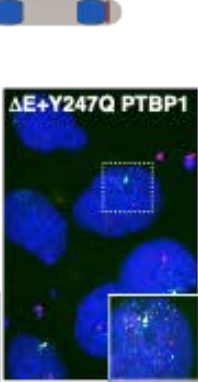

f

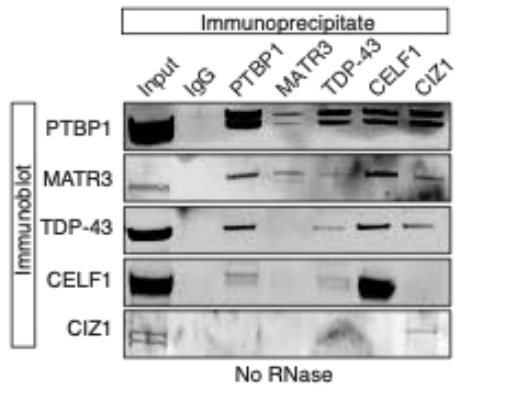


Figure 4: PTBP1, MATR3, TDP-43 and CELF1 confer function on the E-repeat to localize Xist and enable persistent $\mathrm{X}$-linked gene silencing during XCI-initiation

a, Depiction of the MCP-fusion protein rescue strategy for $\Delta \mathrm{E}$ Xist.

b, Illustration of Flag-tagged MCP-fusion proteins used in this study and mutants thereof. Point mutations are indicated with asterisks (see methods for MATR3 PRI mutant sequence, denoted by 3 black asterisks). Amino acid length of fusion proteins includes Flag-tag and MCP sequence.

c, Representative epifluorescence images of RNA FISH against Xist (green) and MS2 (red) in day 7 differentiated WT, $\Delta \mathrm{E}$, and $\Delta \mathrm{E}$ lines expressing variants of Flag-tagged MCP-PTBP1 fusions. Inset: enlargement of MS2+Xist ${ }^{129}$ clouds outlined with hatched lines. Chosen clouds are representative of the $\mathrm{MS} 2+X i s t^{129}$ cloud phenotype in $\Delta \mathrm{E}$ cells upon rescue with the indicated protein variant.

d, Histogram showing the number of nuclei with an Xist cloud that also displayed a co-localizing MS2 signal at differentiation day 7, as detected by RNA FISH, in WT, $\Delta \mathrm{E}$, or $\Delta \mathrm{E}$ lines expressing the indicated Flag-tagged MCP fusions (see methods for information on scoring). 83-103 cells were counted per sample. Error bars represent the SEM from two independent experiments (pvalues: $*<0.05, * *<0.005,2$-tailed students t-test).

e, Histograms showing the quantification of nascent Gpc4 or Atrx nascent expression pattern in cells displaying an MS2 $+X i s t^{129}$ coated X-chromosome at differentiation day 7 as described in (d). 50 cells with an MS2+Xist ${ }^{129}$-coated X-chromosome were counted per sample from one experiment.

f, Immunoprecipitation of PTBP1, MATR3, CELF1, TDP-43 and CIZ1 from ESC extracts (not RNase treated) and detection of co-precipitated proteins by immunoblotting, using the same antibodies. Image is representative of the results obtained from three independent replicates. 
bioRxiv preprint doi: https://doi.org/10.1101/2020.03.09.979369; this version posted March 12, 2020. The copyright holder for this preprint (which was not certified by peer review) is the author/funder. All rights reserved. No reuse allowed without permission.

a

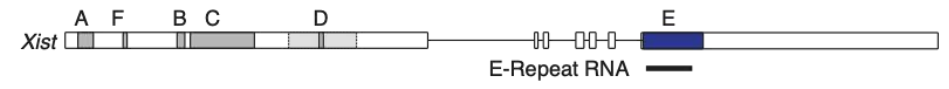

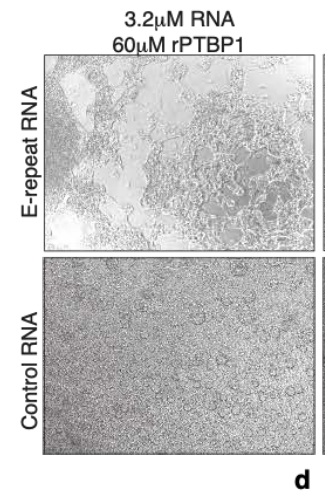
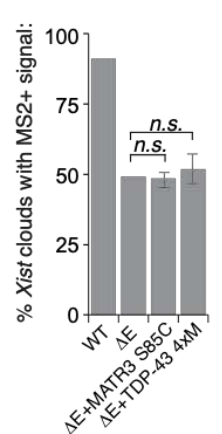
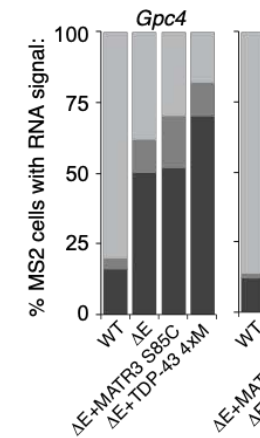

g

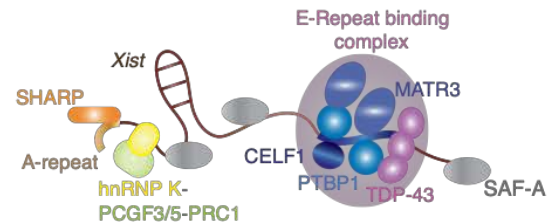

$0.3 \mu \mathrm{M}$ RNA
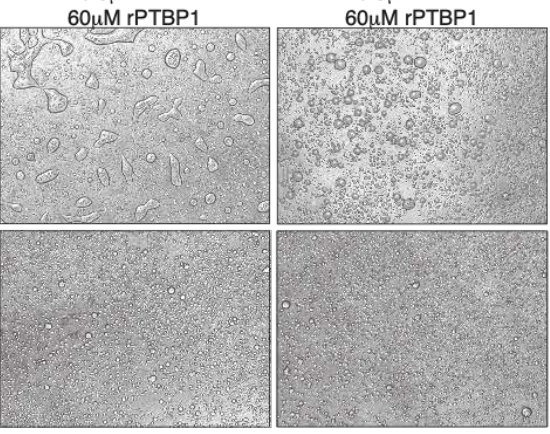

e
$0.1 \mu \mathrm{M}$ RNA
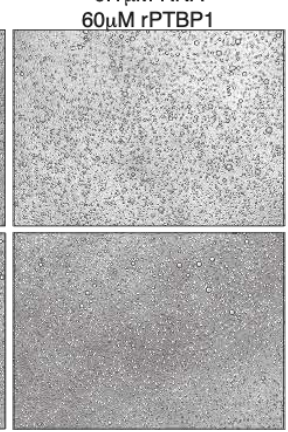

$100 \mu \mathrm{m}$

Xist - -
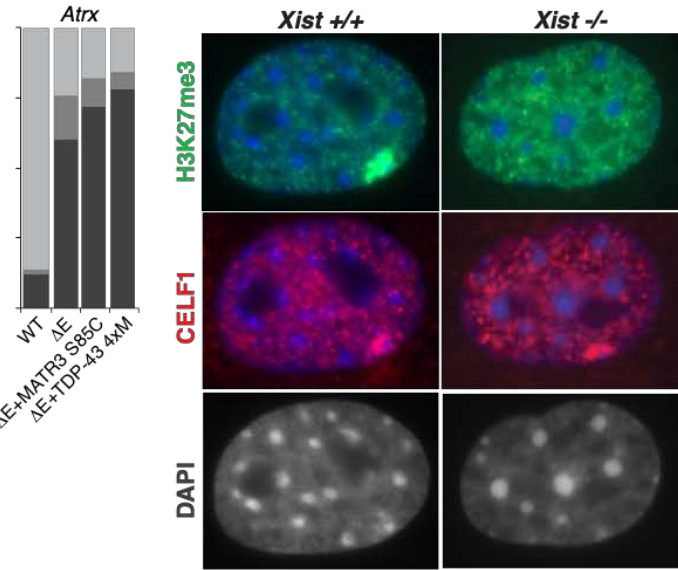

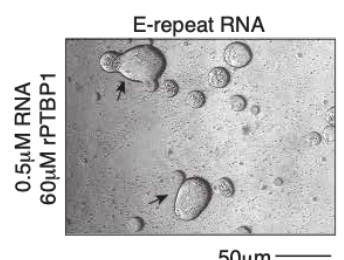

$\mathbf{f}$

h

Initial Xist RNP spread across euchromatic, active $X$
SHARP- and PRC1-dependent $X$-linked gene silencing Increasing Xist RNP abundance
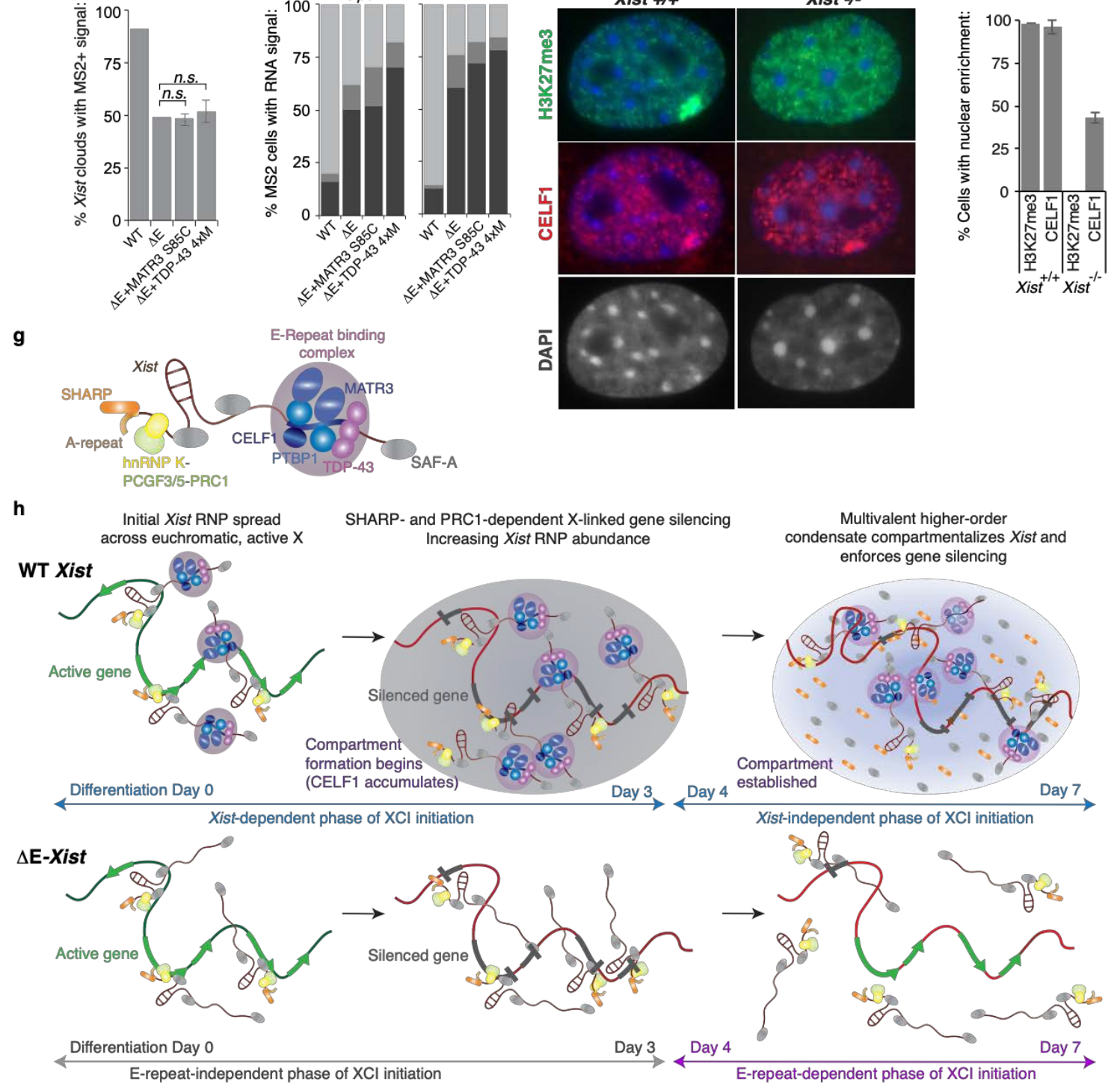
Figure 5: Self-association of E-repeat binding RBPs is critical for compartment formation

a, Top: Diagram of the Xist locus with introns, exons and repeat arrays A-F depicted as in Fig. 2a. The Xist-derived in vitro transcribed E-repeat used in droplet assays is indicated. Bottom: Brightfield (10x) images showing droplets formed after 40 minutes with $60 \mu \mathrm{M}$ rPTBP1 and decreasing concentrations of Xist E-repeat or control RNAs.

b, Brightfield image of droplets formed after 40 minutes with $60 \mu \mathrm{M}$ rPTBP1 and $0.5 \mu \mathrm{M}$ E-repeat RNA, which have fused or are undergoing fusion, indicated by arrows. Images were taken at higher magnification than in (a), note scale bar.

c, Histogram showing the number of nuclei with an Xist cloud that also displayed a co-localizing MS2 signal, as detected by RNA FISH, in WT, $\triangle \mathrm{E}$, or $\triangle \mathrm{E}$ cells expressing Flag-tagged MCPMATR3-S85C and TDP-43 4xM, respectively. 108-144 cells were counted per sample, from 2 independent experiments. Data for WT and $\Delta \mathrm{E}$ samples were from a single experiment but was combined with data from Fig. 2 to calculate experimental standard error of the mean. There was no significant difference between the $\Delta \mathrm{E}$ sample and experimental $\Delta \mathrm{E}$ rescue lines (2-tailed students t-test).

d, Histograms showing the quantification of nascent Gpc4 or Atrx nascent expression patterns in cells described in (c) displaying an MS2+Xist ${ }^{129}$ coated X chromosome at differentiation day 7. 50 cells displaying an MS2+Xist ${ }^{129}$ were counted per sample, from one experiment.

e, Representative fluorescence microscopy images showing H3K27me3 (green) and CELF1 (red)

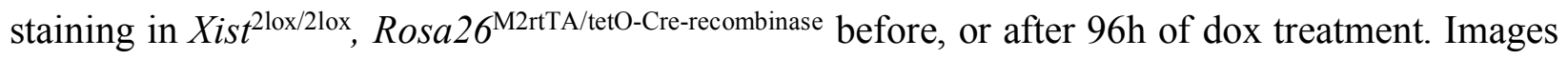
of the DAPI stained nuclei (grey) are shown below.

f, Histogram showing the percentage of cells with H3K27me3 or CELF1 enrichment before or after $96 \mathrm{~h}$ of dox treatment $(2 \mathrm{ug} / \mathrm{mL})$. Error bars represent SEM from two biological replicates.

g, Illustration of the Xist ribonucleoprotein complex. PTBP1, MATR3, CELF1 and TDP-43 bound to the E-repeat are depicted in blue/purple. The A-repeat bound by SHARP is shown in yellow/brown, and hnRNPK-PGCF3/5-PRC1 bound to the B-repeat indicated in yellow/green. Proteins marked in grey indicate additional Xist-interactors, such as SAF-A/hnRNP-U.

h, Model for how the E-repeat forms the Xi domain and facilitates the transition from the Xistdependent to the Xist-independent phases of XCI-initiation. Top: At the onset of differentiation, Xist expression is induced and the Xist RNP spreads across the euchromatic X-chromosome (green line) using the $3 \mathrm{D}$ conformation of the chromosome and mechanisms involving SAF-A/hnRNP U 
to attach to chromatin. SHARP, bound at the A-repeat, recruits HDAC3 (not shown) to initiate transcriptional shutoff for genes. HnRNPK, bound at the B-repeat, instates heterochromatin formation (red line) via deposition of PRC1-dependent H2AK119ub and PRC2-dependent H3K27me3, and contributes to gene silencing. Further differentiation and likely DNA compaction, along with rising Xist levels, increase the overall local concentration of Xist RNPs, such that more Xist RNPs integrate into each Xist granule detected in 3D-SIM experiments. At a critical concentration of Xist transcripts and E-repeat-binding RBPs, the formation of a higher-order multimolecular and multi-valent assembly occurs. This establishes a self-sustaining condensate that compartmentalizes Xist within the Xi-domain and enforces X-linked gene silencing, and that can be sustained in the absence of Xist. Bottom: Without the E-repeat, the Xist-retaining silencing compartment does not form, Xist localization cannot be reinforced, rendering the local concentration of Xist and its directly interacting proteins insufficient to maintain gene silencing, which ultimately results in reactivation of the $\mathrm{Xi}$. 


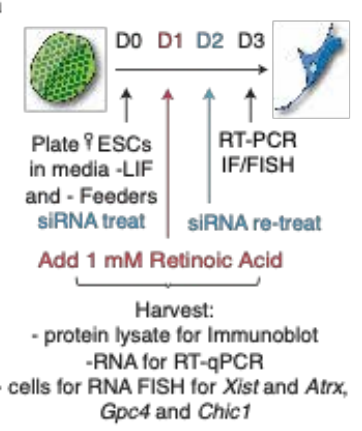

c $\left.\begin{array}{l}\text { Single spot } \\ \text { co-localizing with Xist } \\ \text { Bi-allelic }\end{array}\right] \begin{aligned} & \text { Lack of } \\ & \text { silencing }\end{aligned}$

i

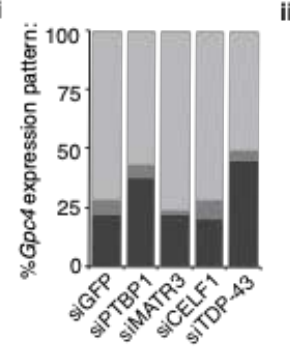

b i

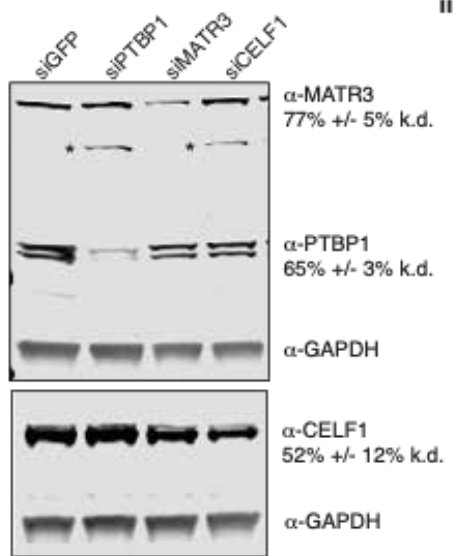

Single spot not co-localizing with Xist

iii
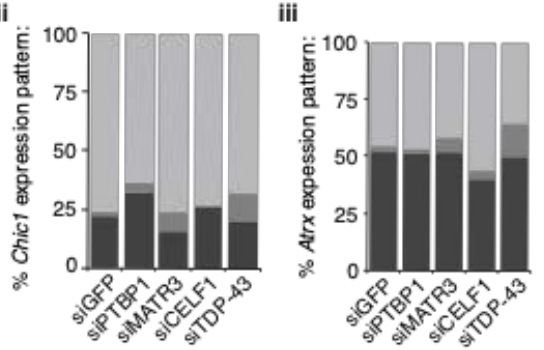

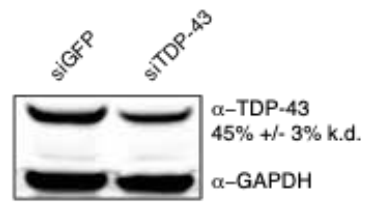

d

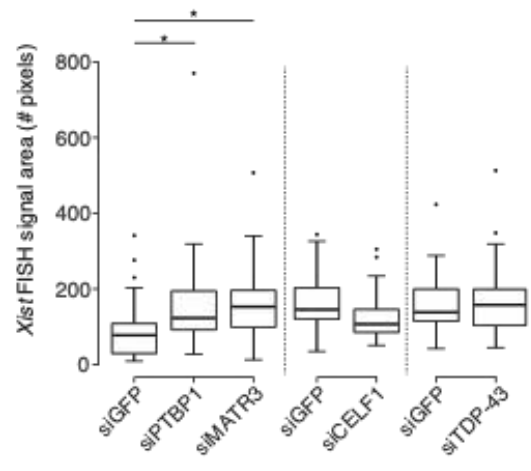

e

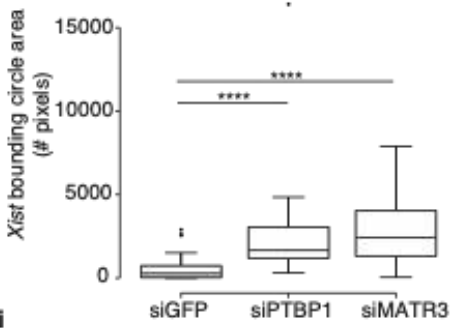

g

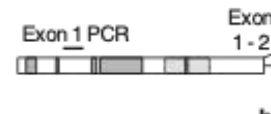

h

Exon 1-2

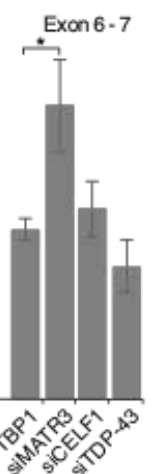

j
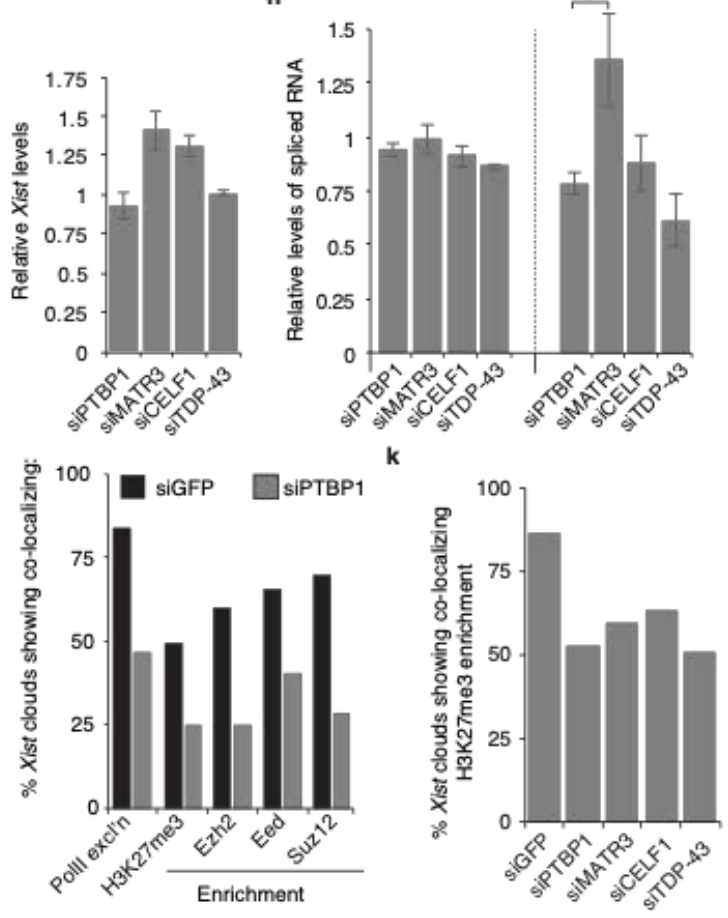

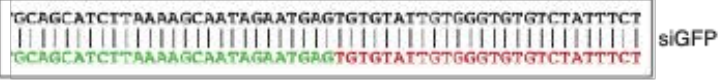

GCAGCATCTTAAAAGCAATAGAATGAGTGTGTATTGTGGGTGTGTCTUTTTCT IIIIIIIIIIIIIIIIIIIIIIIIIIIIIIIIIIIIIIIIIIIII Exon 7 


\section{Extended Data Figure 1: Depletion of PTBP1, MATR3, TDP-43 or CELF1 does not strongly affect $\mathrm{X}$-linked gene silencing or Xist processing}

a, Overview of siRNA approach used to deplete Xist-interacting RBPs in female differentiating mouse ESCs and to assess XCI.

b i, Representative immunoblot showing the level of siRNA-mediated knockdown of PTBP1, MATR3, and CELF1 relative to siGFP treatment in cells differentiated for 3 days. The percentage depletion normalized to GAPDH is shown on the right. Error represents the SEM from 3 independent experiments. ii, As in (i), except for TDP-43.

c i, Histogram showing the nascent expression pattern of X-linked gene Gpc4 after 3 days of differentiation and knockdown of the indicated factor. 50 cells with Xist clouds were counted per sample from one experiment. ii, Same as (i) except showing data for the X-linked gene Chicl. iii, Same as (i) except showing data for the X-linked gene Atrx.

d, Box plots showing the distribution of the area (mask) covered by Xist RNA FISH signal upon knockdown of PTBP1, MATR3, CELF1 or TDP-43, used to calculate the Xist aggregation scores displayed in Figure 1b. (p-value: $*<0.05$, 2-sample KS test). 25-36 clouds were measured per sample, from one experiment.

e i, Box plots showing the distribution of the area of the bounding circle encompassing Xist FISH signal/mask upon knockdown of PTBP1 or MATR3 used to calculate the Xist aggregation scores displayed in Figure 1b. ii, Same as (i) except for knockdown of CELF1 or TDP-43. (p-value: *< $0.05, * * * *<0.00005,2$-sample KS test). 25-36 clouds were measured per sample, from one experiment.

f, Diagram of Xist showing the splicing events assessed upon knockdown of PTBP1, MATR3, TDP-43 or CELF1, and amplicon used to assess Xist abundance (exon 1 PCR). The results are shown in (i) and (j).

g, Histogram showing the relative abundance of total Xist transcripts upon knockdown of indicated RBPs. All samples are normalized to siGFP control treatment samples and Snrnp27 RNA (encodes a U6 snRNP factor). Samples were assessed in triplicate from 3 independent depletions. Error bars represent the SEM.

h, Histogram showing the relative level of Xist exon 1-2 and exon 6-7 splicing upon knockdown of indicated factors, normalized as in (j). Samples were assessed in triplicate from three independent depletions. Error bars represent the SEM (p-value: *<0.05, 2-tailed students t-test). 
i, Snapshot of aligned sequences derived from the expected sequence upon splicing of exon 6 (green) - exon 7 (red) (colored, lower sequence), and the observed sequence upon siRNA treatment targeting GFP or PTBP1 after 72h in differentiating female ESCs (black, upper sequence). Two independent experiments were tested for splicing with identical results showing correct exon 6 - 7 ligation.

j, Histogram showing the percentage of Xist-positive cells showing co-localizing exclusion of RNA-pol II or enrichment of PRC2 components EZH2, EED, SUZ12 and the histone modification $\mathrm{H} 3 \mathrm{~K} 27 \mathrm{me} 3$ on the $\mathrm{Xi}$, in female ESCs differentiated for 3 days and treated with siRNAs against GFP (control) or PTBP1. 54-64 cells were counted per sample from one experiment.

$\mathbf{k}$, Histogram showing the percentage of Xist-positive cells with H3K27me3 Xi-enrichment in day 3 differentiated female ESCs treated with siRNAs against GFP (control), PTBP1, MATR3, TDP43 or CELF1. 109-146 cells were counted per sample, from one experiment independent of that shown for (f). 
bioRxiv preprint doi: https://doi.org/10.1101/2020.03.09.979369; this version posted March 12, 2020. The copyright holder for this preprint (which was not certified by peer review) is the author/funder. All rights reserved. No reuse allowed without permission.

a

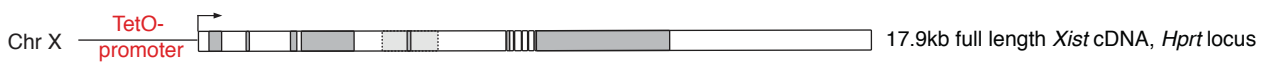

b

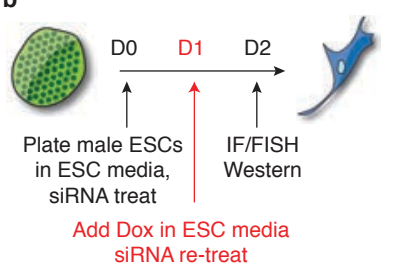

c

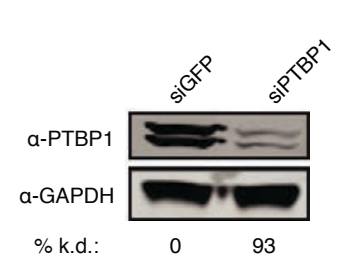

d

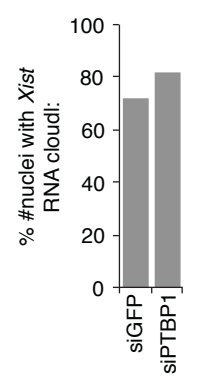

e

tetO-Xist cDNA (17.9kb, Hprt)

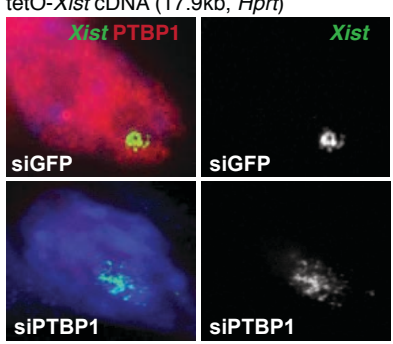




\section{Extended Data Figure 2: Xist dispersal upon PTBP1 knockdown is independent of splicing}

a, Diagram of a tet-inducible full length $(17.9 \mathrm{~kb})$ Xist cDNA transgene inserted into the Hprt locus on the X-chromosome in male ESCs.

b, Approach used to deplete PTBP1 in the cells described in (a).

c, Immunoblot and quantification showing level of PTBP1 knockdown in the experiment diagrammed in (b).

d, Histogram showing the percentage of cells with an Xist RNA cloud upon knockdown of PTBP1 and 24 hours of dox treatment in the experiment as described in (b). $82-90$ cells were counted per sample in one experiment.

e, Representative images of Xist, detected by RNA FISH, after being treated with siRNAs against GFP or PTBP1 and dox-addition, as outlined in (b). Note the dispersal of Xist upon PTBP1 knockdown occurs in the absence of splicing regulation, as Xist is expressed from a cDNA construct in this experiment. 
bioRxiv preprint doi: https://doi.org/10.1101/2020.03.09.979369; this version posted March 12, 2020. The copyright holder for this preprint (which was not certified by peer review) is the author/funder. All rights reserved. No reuse allowed without permission.

a

\begin{tabular}{|l|c|c|c|c|c|}
\hline PTBP1 CLiP & \# reads in Dataset & Window & \# Reads in window & \% of total mapped reads & \% of reads in Xist \\
& $3,328,965$ & 1st kb of Xist (1000nt) & 1084 & 0.0325 & 0.897 \\
\hline & & E repeat (1435nt) & 111514 & 3.349 & 3.629 \\
\hline & Xist (22860nt) & 120827 & & 92.292 \\
\hline & & & & 0.028 & 1.058 \\
\hline MATR3 CLiP & $5,020,287$ & 1st kb of Xist & 1425 & 2.482 & 92.579 \\
\hline & & E repeat & 124617 & 2.681 & \\
\hline & $3,897,448$ & Xist & 134605 & & 0.004 \\
\hline
\end{tabular}

b TGTGTATTGTGGGTGTGTCTATTTCTTGTTTTATGTATCTATTTTTTCCTTGGTCTGTGTGTCTAATTCTTTGTTACATC TATTTCTTCCTTGCTTTGTGTGTCTATTTCTTCCTTGCTTTGTGTGTCTATTTCTTCCTTGCATTATGTCTAATTCTTTG TTATATCTATTTCTTCCTTGCTTTGTGTCTATTTCTTCCTTGCAGTTGTGTCTAATTCTTTGTTACATCTATTTCTTCCT TGCTTTGTGTGTCTATTCTTCCTTGCATTGTGTCTAATCTTTGTTATATCTATTTCTTCCTTGCTTTGTGTGTCTGTC TTCCTTGCTTTGTGTCTATTTCTTCCTTGCAGTTGTGTCTAATCTTTGTTACATCTATTTCTTCCTTGCTTTTGTGTGT СTTTCTTTCTTGCTTTTGTGTGTCTATTTCTTCCTTGCAGTTGTGTCTAATTCTTTGTTACATCTATTTCTTCCTTGCTT TTGTGTGTCTATTTCTTCCTTGCATTGTGTCTAATTCTTTGGTATATATATTTCTTCATTGCTTTGTGTGTCTATGTCTC CTTGTGTTGTCTAATCGTTGTTGCATCTATTTCTTCCTTGCTTTGTGTGTCTATTTCTTCCTTGCTTTGTGTGTCTATG TCTTCCTTGCTTTGTGTGTCTATGTCTTCCTTGTTTTGTGTATCTACTTCTTCCTTGTGTGTCTAATTCTTTGTTACATC TATTCTTCCTTCCTTTGCATGTCTCCTTCTTTCCTTTGTGTGTCTTTTCTGTCTGCAGTGTGTCTTACCTATTCCCATG TTTCTCCTGCATGTTCTTTCTTGCAGAGCTTTGAGCTTTGTTTCACTTTCTCTGGTGCCTGTGTGGTCTGCTTTGTCTTC ACTAGCTATGGCTCTCTGTTTTATCTATCTGGTtGCTATTTCTCTTAGCTTTTCTTTCACTCCTGCCTTTCGTGACTCCC CTTTGGGTCACATGTTGCATGCATCCCTCTCTTTTTCTTGTGCTCACCCCACTTGTTCTTTGTTCAAGTTCTCTTTGTCA GTCCATTTCAGTTTTCTTTCTGCTGCTTCTATCCTTAGTGAATCTTGTTTACATTTCTTCCCTGCCTTTCTTGGGCCAC TTTCTCTGTTTTCTTTTGTATTTGTGTCTCTTTGCTATTGGTGGATTTCTTATCTCAGCATCATTCTGTTGCTTTGTGTT TGCTTGTGTTTCTATCTTCTACTTTCCTCCTTTCTGTTCACTTTGAGCATTTCATCTCTTTACAAGTCTGTGTCTCTCTT GTATTCTAAAGTAATCCTTTCTTGGATGTTTCTTTGTATGTACATGTGCGTGTGTGCATGTGTGTTATGTGTGTCATGTG TGAGAGGAGCTTCATAGCCCCTTCCCAATAGGTCCAGAATGTCACCCGTGGAGCCGTTCCTCACACCAGACTGCCCTGAG AAATAATCTAAGACAAAATACATCATTCCGTCCGGTCAG GATTCAAGTGG
${ }^{*}$ Xist gene length

c

\begin{tabular}{|c|c|}
\hline 1 . & TTTCTTCCTTGCTTTGTGTGTCTA \\
\hline 2 & TTTCTTCCTTGCTTTGTGTGTCTA \\
\hline 3 & TTTCTTCCTTGCTTTGTGTGTCTA \\
\hline 4 . & TTCTTCCTTGCATTATGTCTA \\
\hline 5 . & TTTCTTCCTTGCTTTGTGTCTA \\
\hline 6 . & TTTCTTCCTTGCAGTTGTGTCTA \\
\hline 7 . & TTTCTTCCTTGCTTTGTGTGTCTA \\
\hline 8 . & TTTCTTCСTTGCATTGTGTCTA \\
\hline 9 . & TTTCTTCCTTGCTTTGTGTGTCTG \\
\hline 10 . & TCTTCCTTGCTTTGTGTCTA \\
\hline 11. & TTTCTTCCTTGCAGTTGTGTCTA \\
\hline 12 . & TTTCTTCCTTGCTTTTGTGTGT \\
\hline 13. & СтTTCTTTCTTGCTTTTGTGTGTCTA \\
\hline 14 . & TTTCTTCCTTGCAGTTGTGTCTA \\
\hline 15 . & TTTCTTCCTTGCTTTTGTGTGTCTA \\
\hline 16 . & TTTCTTCCTTGCATTGTGTCTA \\
\hline 17. & TTTCTTCATTGCTTTGTGTGTCTA \\
\hline 18. & TCTCCTTGTGTTGTCTA \\
\hline 19. & TTTCTTCCTTGCTTTGTGTGTCTA \\
\hline 20. & TTTCTTCCTTGCTTTGTGTGTCTA \\
\hline 21 . & TCTTCCTTGCTTTGTGTGTCTA \\
\hline 22 . & TCTTCCTTGTTTTGTGTATCTA \\
\hline 23 & TTCTTCCTTGTGTGTCTA \\
\hline 24 . & TCTCCTTCTTTCCTTTGTGTGT \\
\hline 25 . & ГTTCTGTCTGCAGTGTGTCTTACC \\
\hline
\end{tabular}

d 


\section{Extended Data Figure 3: A tandem array of 20-25nt C/U/G-rich elements comprises the Xist E-repeat}

a, Table showing mapping statistics of PTBP1, MATR3 and CELF1 CLIP-seq data for male ESCs with a tet-inducible Xist allele, induced for 6 hours, for reads mapping across the Xist locus, the Erepeat of Xist, and the $1^{\text {st }} \mathrm{kb}$ of Xist (including the A-repeat) as a percentage of total mapped reads or of the reads located within Xist. Note that Xist is overexpressed in this experiment, which influences the total number of reads mapping to the locus.

b, The first 1500nt of the terminal exon 7 of Xist are shown. The sequence remaining after splicing of the Xist $\Delta \mathrm{E}$ transcript is underlined (see Extended Data Figure 6). The $\mathrm{C} / \mathrm{U} / \mathrm{G}$ tandem repeats within the 5' half of the E-repeat are indicated (pink - full repeats, blue- truncated repeats) as are the CU-tracts (shown as CT tracks in the DNA sequence, green) in the 3' half of the sequence. Potential TDP-43 sites are shown in orange at the 3' terminal end of the E-repeat.

c, Alignment of the 25 full C/U/G-tandem repeats (pink) in (b). Brown, green, red and black text indicate similar nucleotide runs within each tandem repeat. Brown $\mathrm{C} / \mathrm{U}$ tracts encode putative PTBP1 and/or MATR3 binding sites. Red G/U tracts encode putative CELF1 and/or TDP-43 binding sites.

d, Alignment of the 9 truncated C/U/G-tandem repeats (blue) in (b)). Orange nucleotides are variable within each truncated repeat unit. 
bioRxiv preprint doi: https://doi.org/10.1101/2020.03.09.979369; this version posted March 12, 2020. The copyright holder for this preprint (which was not certified by peer review) is the author/funder. All rights reserved. No reuse allowed without permission.

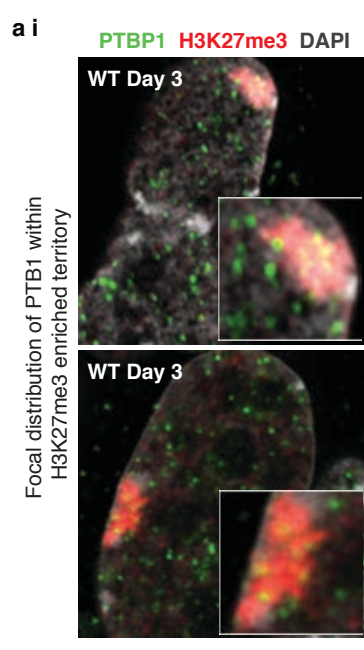

b i

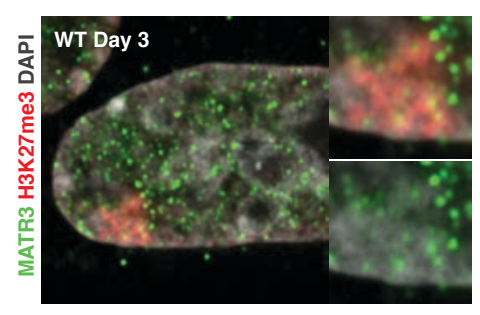

c i

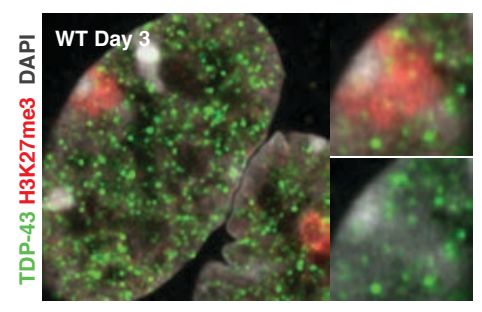

ii

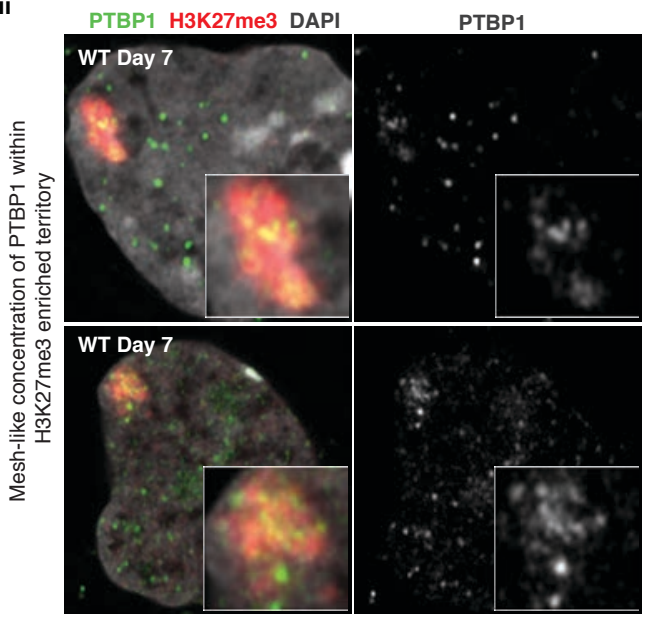

ii

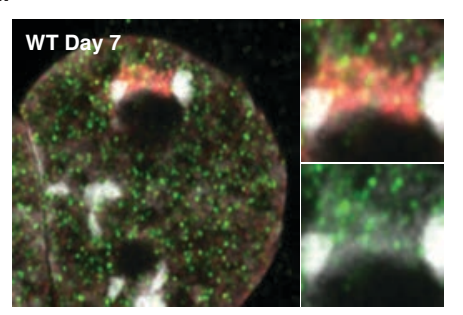

ii

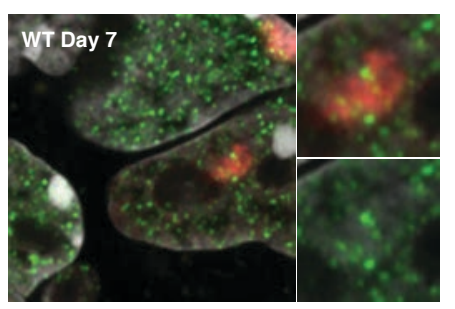




\section{Extended Data Figure 4: PTBP1 concentrates in a unique pattern within the Xist-coated X- chromosome territory at day 7 of XCI initiation}

a i, Left: Two independent micrographs of confocal-airyscan optical sections through nuclei of WT female ESCs at day 3 of differentiation immunostained for PTBP1 (green) and H3K27me3 (red, detecting the Xi) and counterstained with DAPI (grey). Inset: enlargement of the Xi-territory, showing a punctate, focal pattern of PTBP1 within the Xi. Right: same as left, except showing only the PTBP1 signal in grayscale. ii, Same as in (i), except showing WT female ESCs differentiated for 7 days, showing a mesh-like PTBP1 concentration within the Xi territory that is distinct from that observed in the nucleoplasm of these cells or from the pattern within the day 3 $\mathrm{Xi}$.

b i, Confocal-airyscan optical sections through nuclei of WT female ESCs at day 3 of differentiation immunostained for MATR3 (green) and H3K27me3 (red) and counterstained with DAPI (gray). Right: enlargement of the Xi territory showing MATR3 and H3K27me3 signals (upper) or only MATR3 signal (lower) with DAPI counterstaining (gray). ii, Same as in (i), except showing cells differentiated for 7 days.

c i, Same as in (b-i) except showing immunostaining for TDP-43 at day 3 of differentiation. ii, Same as in (b-ii) except showing immunostaining for TDP-43 at day 7 of differentiation. 
bioRxiv preprint doi: https://doi.org/10.1101/2020.03.09.979369; this version posted March 12, 2020. The copyright holder for this preprint (which was not certified by peer review) is the author/funder. All rights reserved. No reuse allowed without permission.

a

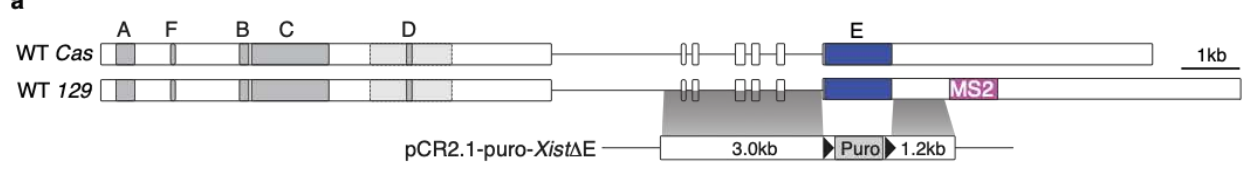

b

$17.5 \mathrm{~kb}$

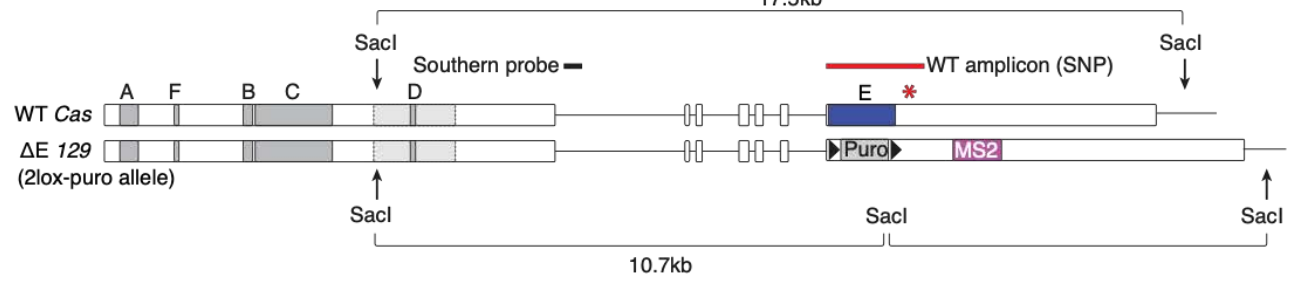

c

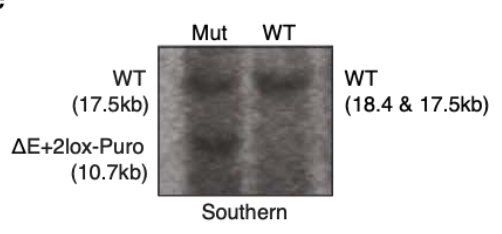

e

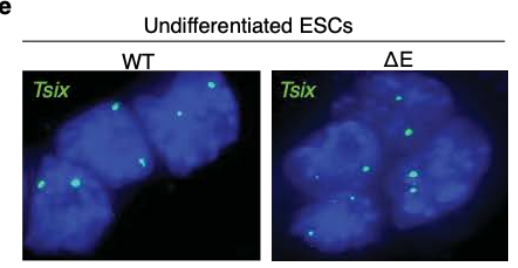

Amplicon sequence 129 allele sequence TCAGAATTCAAGTGGTCTGAagtGaACGCCCAagtagaAgacagaAGTTTTGCGaCtTG AGATTTAAAAGGACCAAAATACACAGATGGCCCATCTTGAGCTGGCTGGACAGAATGCTG

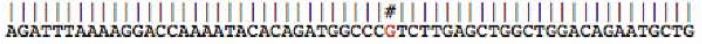
ACAACCCAAAGAAGAGGAACTGTTTCTACAGGACACCTGTGACTTCCAAGAGCGGAGAAC

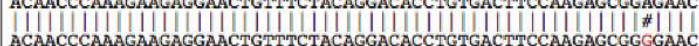

f

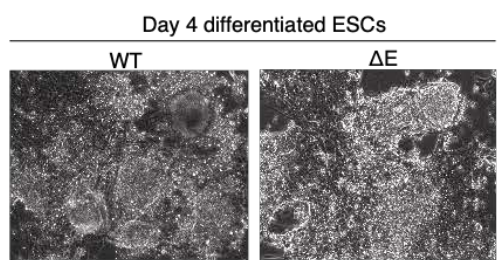

g

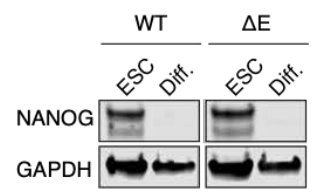




\section{Extended Data Figure 5: $\Delta \mathrm{E}$ cells undergo differentiation similar to WT cells}

a, Scale diagram of the Xist gene annotated with repeat regions A-F (see Figure 2a for complete description). Also shown is the targeting strategy used to delete the Xist E-repeat region in F1 2-1 MS2 female ESCs with the targeting plasmid pCR2.1-Puro-Xist $\Delta \mathrm{E}$.

b, Diagram of the southern blot strategy with a 5' external probe, used to identify deletion clones. This strategy did not distinguish between targeting of the E-repeat on the 129 or cas allele of Xist. Location of southern probe is shown, as is the location of the PCR amplicon (red) used to identify which allele was deleted (see (d)). Asterisk denotes location of SNPs used for defining the allelic origin of the deleted allele (see (d)).

c, Southern blot as described in (b) on targeted female ESCs with a loxP-flanked puromycin cassette in place of the E-repeat on one Xist allele. The puromycin cassette was subsequently deleted by Cre-recombinase transfection into the mutant line and confirmed by PCR after subcloning.

d, Sequencing analysis of the long amplicon (shown in b). SNPs conforming to the 129 allele are shown in red and are not detected in the amplicon, confirming that the E-repeat remains present on the Cas allele and is deleted on the 129 allele.

e, Tsix RNA FISH (green) on undifferentiated WT and $\Delta \mathrm{E}$ cells confirming the presence of two Tsix nascent transcription units, used as a proxy to confirm that the targeted $\Delta \mathrm{E}$ cells maintain two X-chromosomes. DAPI stained nuclei are shown in blue.

f, Brightfield images of WT and $\Delta \mathrm{E}$ cells at day 4 of differentiation, showing that the cells do not appear morphologically different upon induction of differentiation.

g, Immunoblot of lysates from WT and $\Delta \mathrm{E}$ cells at 2 days of differentiation showing early and equal loss of Nanog expression, a pluripotency gene quickly down-regulated with the induction of differentiation. 
bioRxiv preprint doi: https://doi.org/10.1101/2020.03.09.979369; this version posted March 12, 2020. The copyright holder for this preprint (which was not certified by peer review) is the author/funder. All rights reserved. No reuse allowed without permission.

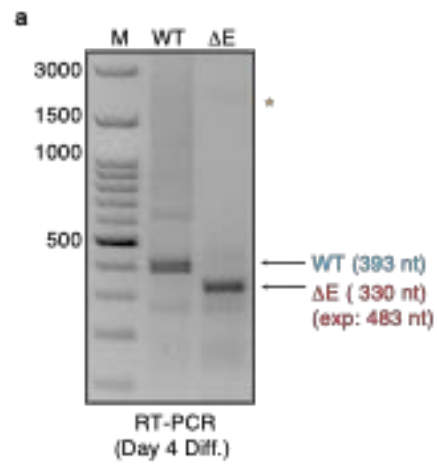

d b

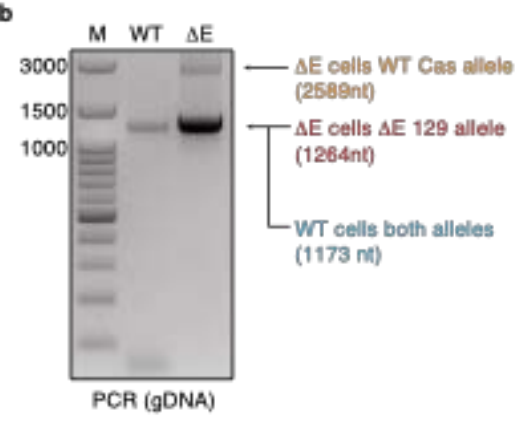

c
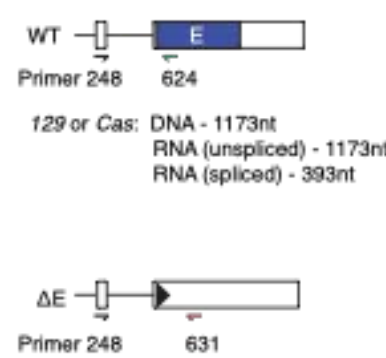

Cas: DNA - 2589nt

RNA (unspliced) - 2589nt

RNA (spliced) - 1655nt

$129 \triangle \mathrm{E}:$ DNA - 1264nt

RNA (unspliced) - 1264nt

RNA (spliced) - 483nt (expected)

RNA (spliced) - 330nt (cbserved)

$\triangle E$ Xist DNA

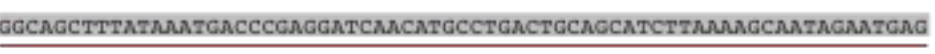

Xist Exon 6

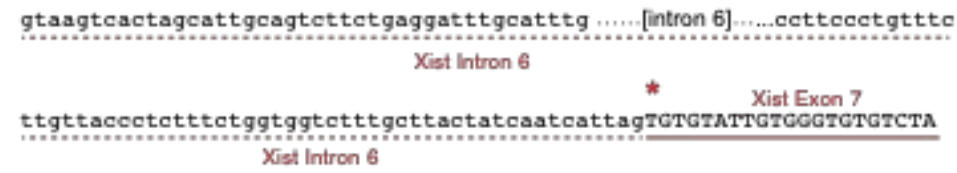

ArCTGCAслатTCGCCCrrGCGGCCGCataacttegtataatgtatgetatacgaagttat

Targeting Vector Sequence LoxP sequence

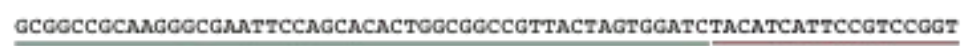

$\star$

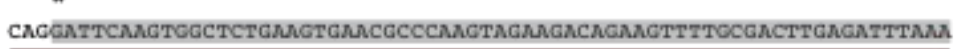

\section{Xist Exon 7}

- Normal $3^{\prime}$ splice sile in WT Xist - skipped in $\Delta E$ Xist transcripts

- Cryptic 3' splice site - used in SE Xist transcripts

AE Xist RNA 


\section{Extended Data Figure 6: Splicing of intron 6 proceeds in the absence of the E-repeat}

a, RT-PCR amplicons generated from RNA isolated from day 4 differentiated WT (with primers APJ248/624) or $\Delta \mathrm{E}$ (with primers APJ248/631) cells across exon 6 - exon 7 to confirm splicing of intron 6. (see (c) for primer locations). We found that that the PCR amplicon was shorter than expected for the $\Delta \mathrm{E}$ transcript. Sequencing across the modified exon 6 - 7 junction revealed the use a cryptic 3' splice site downstream of the LoxP site which extended the E repeat deletion within the Xist transcript (but not the Xist genomic DNA) (as designed) by 42nt. This caused the LoxP site and associated vector sequences to be excluded from the mature $\Delta \mathrm{E}$ Xist transcripts, such that the 3' terminus of exon 6 splices to nucleotide 1479 of exon 7 to form a scar-less junction (see (d)). Orange asterisk indicates a faint band at the expected location for the amplicon generated from the WT Cas allele in $\Delta \mathrm{E}$ cells.

b, PCR amplicons from WT or $\triangle \mathrm{E}$ genomic DNA using the same primers as in (a) showing that the intron 6-containing products can be amplified and thus, non-detection of intron 6-containing Xist transcripts is due to splicing of $\Delta \mathrm{E}$ Xist and not amplification problems.

c, Schematic outlining primers used to assess Xist gDNA and RNA in (a) and (b) across the exon 6 / intron 6 and exon 7 region in WT and $\Delta \mathrm{E}$ cells that have been differentiated for 4 days.

d, Diagram of sequenced genomic and cDNA amplicons (using APJ248/631) confirming correct integration of the targeting plasmid and cre recombinase-mediated removal of the puromycin cassette as well as the sequence of the spliced Xist $\Delta \mathrm{E}$ transcript from exon 6 to exon 7 . Red asterisk denotes wild-type $3^{\prime}$ splice site on exon 7 . Black asterisk denotes splice site used in in $\Delta \mathrm{E}$ transcripts. 
bioRxiv preprint doi: https://doi.org/10.1101/2020.03.09.979369; this version posted March 12, 2020. The copyright holder for this preprint (which was not certified by peer review) is the author/funder. All rights reserved. No reuse allowed without permission.

$\mathbf{a}$

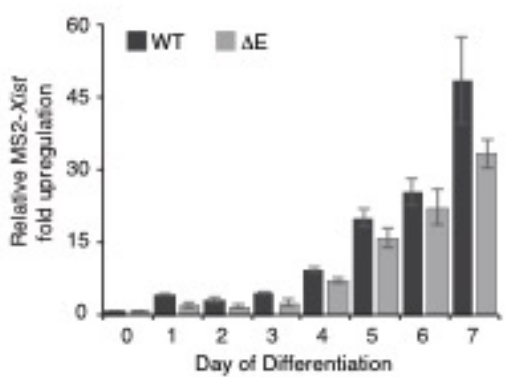

b

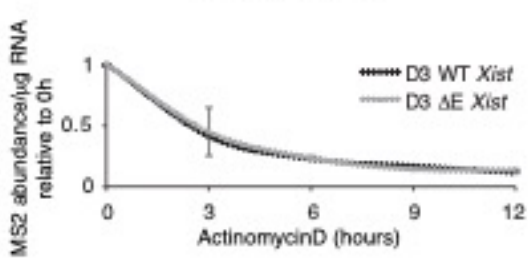




\section{Extended Data Figure 7: Deletion of the E-repeat does not affect Xist abundance or stability}

a, Histogram showing RT-qPCR quantification of the fold upregulation of MS2+Xist ${ }^{129}$ RNA in WT and $\Delta \mathrm{E}$ cells over 7 days of differentiation, normalized relative to undifferentiated samples and against an internal control (Rrm2). Error bars represent the standard error of the mean for three independent samples measured in triplicate. Differences were not significant by 2-tailed students t-test.

b, Line plot showing RT-qPCR measurements of Xist RNA half-life in in WT and $\Delta \mathrm{E}$ cells treated for 3, 6, 9 or $12 \mathrm{~h}$ with ActinomycinD at day 3 of differentiation. Xist RNA amounts were calculated as transcript copy number per microgram of total RNA. Note that $\Delta \mathrm{E}$ Xist RNA displays a similar stability as WT Xist at day 3 of differentiation, which is before the major XCI phenotype in $\Delta \mathrm{E}$ cells manifests, indicating that the deletion of the E-repeat does not make Xist inherently less stable. Samples measured in triplicate from 3 independent samples. Error bars indicate the SEM. 

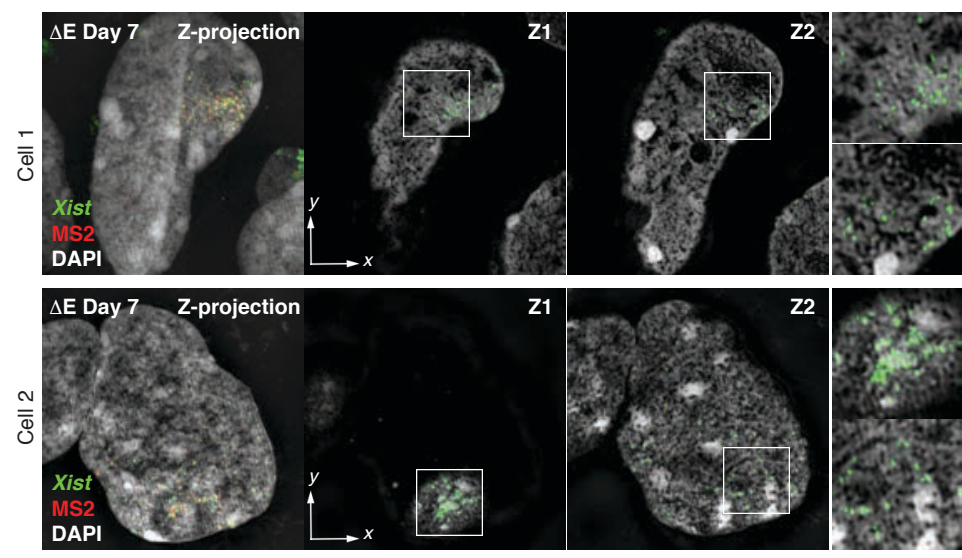

d

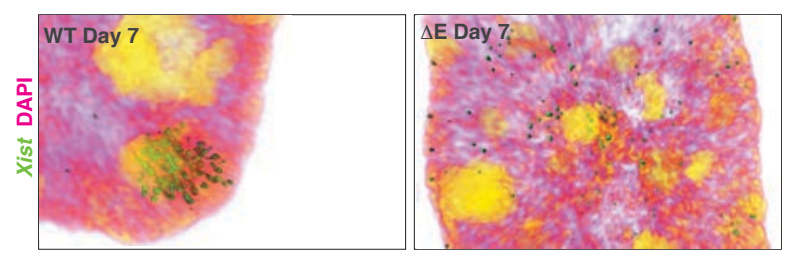

e

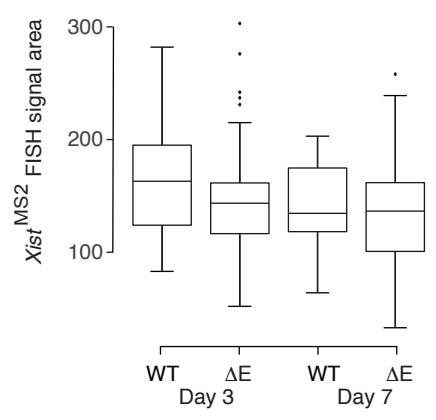

f

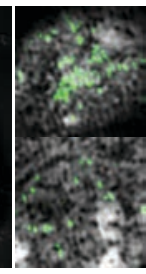

b
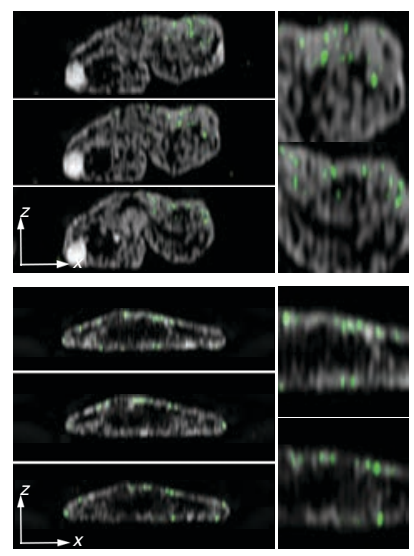

Xist DAPI

MS2 DAPI
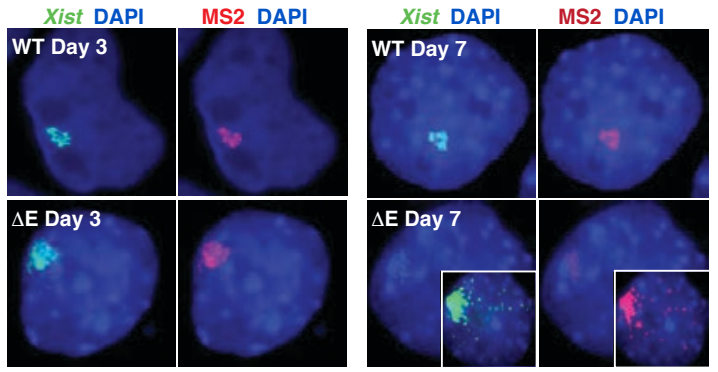

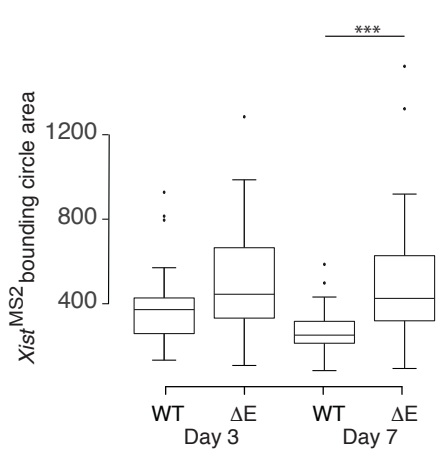




\section{Extended Data Figure 8: $\Delta \mathrm{E}$ Xist transcripts migrate to the nuclear lamina at late stages of XCI-initiation}

a, Left: MIP of 3D-SIM optical sections across a nucleus in $\Delta \mathrm{E}$ cells at day 7 of differentiation hybridized with Xist and MS2 probes. Xist is shown in green, MS2 in red and DAPI in gray. Next images to the right: Two different lateral sections $(Z 1, Z 2)$ through the same nucleus. Far Right: Magnifications of marked regions corresponding to the Xist-MS2-coated X-chromosome from each plane (Z1, top; Z2, bottom). Two independent nuclei are shown in addition to the cell shown in Figure $2 \mathrm{~h}$. Scale bar: $5 \mu \mathrm{m}$.

b, Left top to bottom: consecutive axial cross-sections of the nuclei shown in (a). Right: Magnification of regions with the Xist RNA FISH signal in the top and bottom left panels. Scale bar: $5 \mu \mathrm{m}$. Note the aberrant localization of Xist signal at the nuclear lamina in $\Delta \mathrm{E}$ cells.

c, 3D Amira reconstructions of the Xist-MS2-coated/expressing X-chromosome and surrounding nuclear region in WT and $\Delta \mathrm{E}$ cells shown in Figure $2 \mathrm{~h}$ Xist is shown in green, DAPI with intensity rendering (white/low to orange/high).

d, Representative conventional epifluorescence images of RNA FISH against Xist (green) and MS2 (red) in day 3 and 7 differentiating WT and $\Delta \mathrm{E}$ cells. Chromatin staining with DAPI is shown in blue. Inset: Enhanced image of the Xist/MS2 RNA FISH signal in $\Delta \mathrm{E}$ cells at Day 7. Epifluorescent images are shown for comparison to super-resolution images shown in Figure 2e/h and (a).

e, Box plot showing the distribution of the area (in pixels) covered by Xist RNA FISH signal at day 3 and 7 of differentiation in WT and $\Delta \mathrm{E}$ cells, for MS2 $+X i s t^{129}$ clouds, used for the calculation of the Xist aggregation score in Figure 2f. 30-34 cells were counted per sample from one experiment. 2-sample KS test revealed no significant differences between samples.

f, Box plot showing the distribution of the area (in pixels) of the bounding circle encompassing Xist RNA FISH signal at day 3 and 7 of differentiation in WT and $\Delta \mathrm{E}$ cells, for MS2+Xist ${ }^{129}$ clouds, used for the calculation of the Xist aggregation score in Figure 2f. 30-34 cells were counted per sample from one experiment (p-value: $* * * *<0.00005,2$-sample KS test). 
a
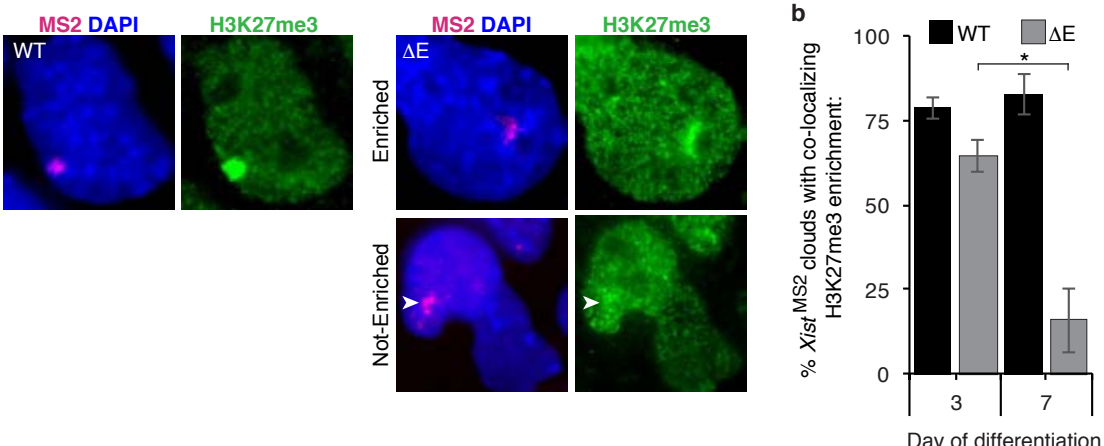

c i
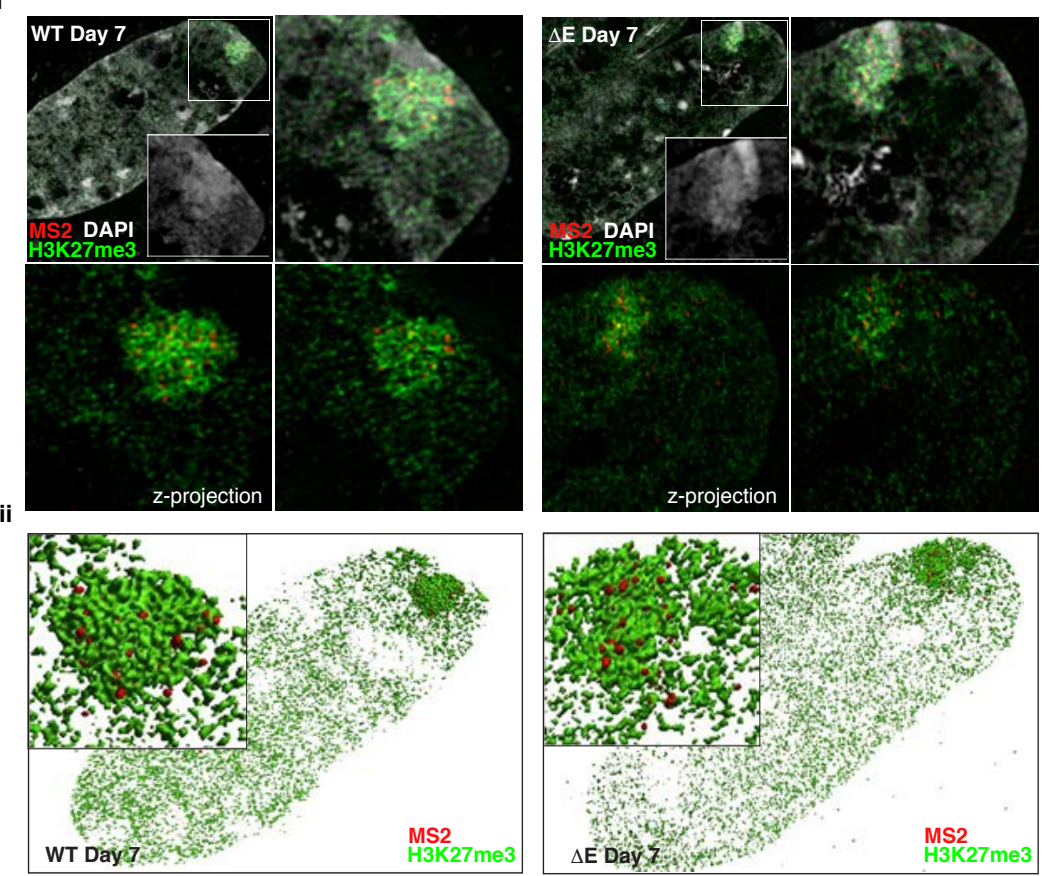


\section{Extended Data Figure 9: At later stages of XCI initiation, the H3K27me3 accumulation on the $\Delta \mathrm{E}$ Xist-coated $\mathrm{X}$-chromosome is less compact than in WT cells}

a, Epifluorescence images of WT and $\Delta \mathrm{E}$ cells at day 7 of differentiation, stained for H3K27me 3 (green), a well-established heterochromatin marker of the Xi, and probed for MS2 by RNA FISH (red). In WT cells, nearly all cells displayed H3K27me3 Xi-enrichment, whereas $\Delta \mathrm{E}$ cells often lacked this Xi-enrichment when Xist-MS2 was expressed. Arrowheads indicate expected position of MS2 co-localizing H3K27me3 immunofluorescent signal.

b, Histogram showing the number of cells with an MS2+Xist RNA FISH cloud that also displayed a co-localizing accumulation of H3K27me3 at days 3 and 7 of differentiation in WT and $\Delta \mathrm{E}$ cells. 60 cells with $X i s t^{\mathrm{MS} 2}$ signal were counted per sample, from 3 coverslips over 2 independent experiments. (p-value: $*<0.05,2$-sample students t-test). To discern enrichment, we first screened cells using the H3K27me3 channel to identify potential H3K27me3 Xi-enrichment, which was then confirmed by checking for a co-localizing MS2 FISH signal. An inability to confidently discern the Xi prior to confirmation with MS2 co-localization was scored as not enriched.

c i, Top left: Lateral 3D-SIM optical sections through the nucleus of day 7 differentiating WT (left) and $\Delta \mathrm{E}$ (right) cells stained for H3K27me3 (green) and hybridized with MS2 probes (red). Chromatin counterstaining with DAPI is shown in grey. Inset: DAPI staining of H3K27me3enriched region corresponding to the Xist-coated X-chromosome. Right: Magnification of inset area showing the H3K27me3, MS2 and DAPI staining (top right) or without DAPI (bottom right). Bottom left: Z-stack MIP of the same cell shown in the bottom right, allowing a comparison between z-stack projection or single optical section of the magnified inset area showing H3me3K27 (green) and MS2 (red) signals. ii, 3D Amira reconstruction of the data shown in (i). H3K27me3 shown in green, MS2 in red. Note less dense packaging of super-resolved H3K27me3 segments around dispersed Xist MS2 signal in the $\Delta \mathrm{E}$ nucleus compared to WT, suggestive of chromatin de-compaction as shown in Extended Data Figure 10 with DAPI staining. 
bioRxiv preprint doi: https://doi.org/10.1101/2020.03.09.979369; this version posted March 12, 2020. The copyright holder for this preprint (which was not certified by peer review) is the author/funder. All rights reserved. No reuse allowed without permission.

a
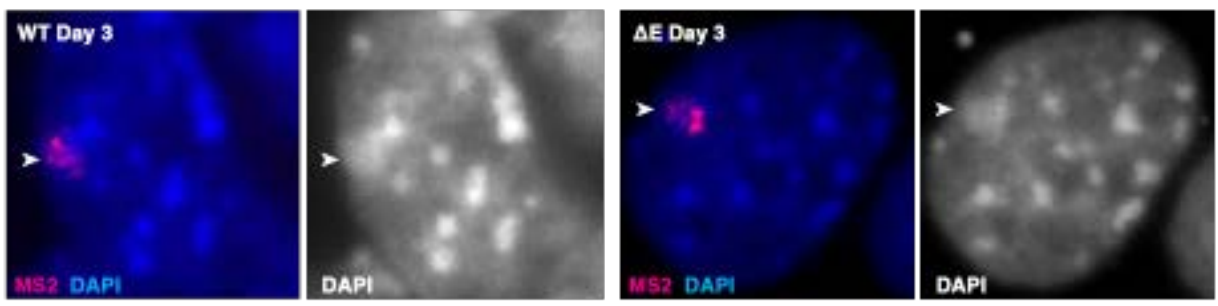

b

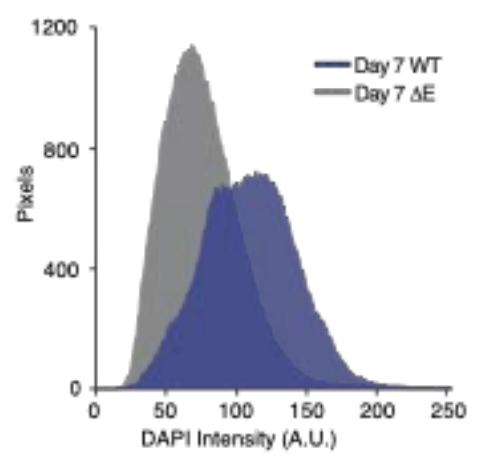




\section{Extended Data Figure 10: DAPI staining intensity of the $\Delta \mathrm{E}$ Xist-coated X-chromosome decreases compared to WT cells at day 7 of differentiation and XCI-initiation}

a, Representative epifluorescence images of day 3 differentiated WT and $\Delta \mathrm{E}$ cells, probed for the MS2 RNA tag by RNA FISH (red). DNA was stained with DAPI (displayed in blue (left) and in grey (right)). Arrowheads point to location of the Xist cloud, highlighting the DAPI-intense staining for WT and $\Delta \mathrm{E}$ Xist-coated X-chromosomes at day 3 of differentiation.

b, Line plot showing DAPI fluorescence intensity within the MS2-Xist-expressing X-chromosome masked by H3K27me3 enrichment in WT (blue) and $\Delta \mathrm{E}$ (gray) cells, differentiated for 7 days. 10 cells were analyzed per cell line from one experiment. These data indicate that the loss of $\Delta \mathrm{E} X i s t$ late in differentiation is accompanied by the loss of intense DAPI staining, a hallmark of the Xi. 
bioRxiv preprint doi: https://doi.org/10.1101/2020.03.09.979369; this version posted March 12, 2020. The copyright holder for this preprint (which was not certified by peer review) is the author/funder. All rights reserved. No reuse allowed without permission.

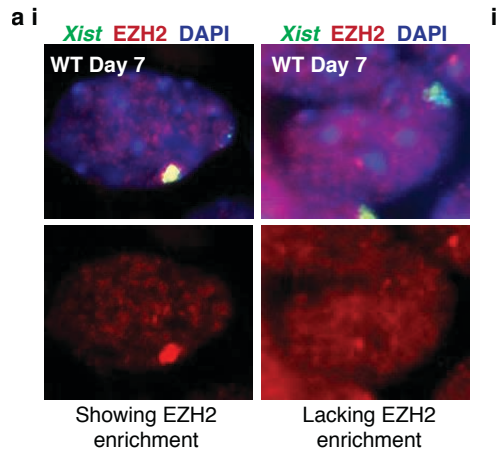

b

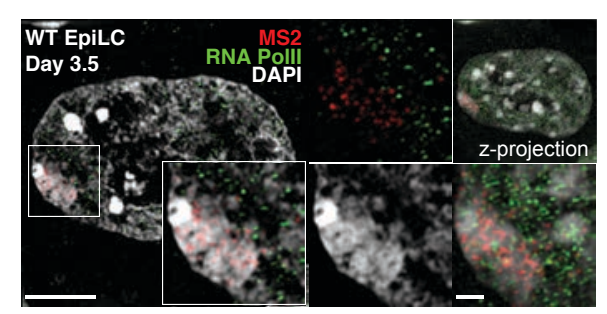

c

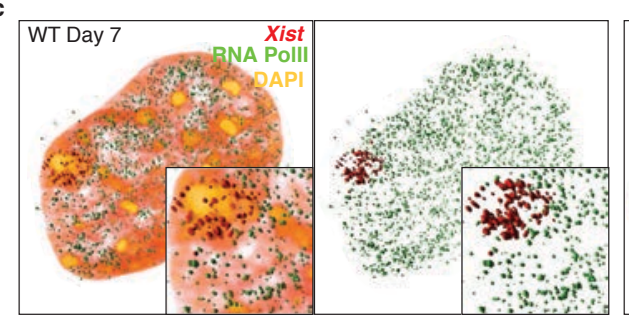

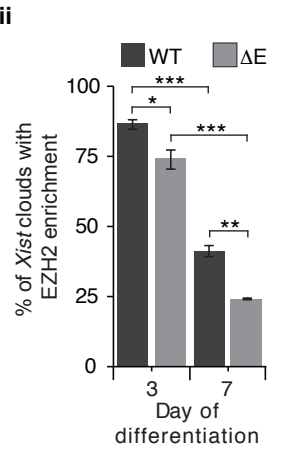




\section{Extended Data Figure 11: EZH2 accumulation and RNA PolII exclusion occurs on the $\Delta \mathrm{E}-$ Xist-coated X-chromosome}

a i, Epifluorescence images of WT female ESCs at day 7 of differentiation stained for EZH2 (red) and probed for Xist by RNA FISH (green), showing cells with (left) and without (right) EZH2 Xienrichment.

a ii, Histogram showing the percentage of Xist clouds with a co-localized EZH2 enrichment in WT and $\Delta \mathrm{E}$ cells at day 3 and 7 of differentiation. 60-70 Xist clouds were counted per sample, from 3 coverslips from 2 independent experiments. (p-value: $*<0.05, * *<0.005, * * *<0.0005,2$-sample students t-test). Note that the E-repeat loss-of-function phenotype arises as the EZH2 enrichment on the WT X-chromosome declines.

b, Lateral 3D-SIM optical sections through the nuclei of WT and $\Delta \mathrm{E}$ female cells at an early stage of XCI-initiation (epiblast-like cells (EpiLCs) at day 3.5 of differentiation from ESCs) stained for RNA-pol II (green) and probed for Xist (red). Chromatin counterstaining with DAPI is shown in grey. Inset: RNA-pol II and Xist signals derived from the single optical section through the Xistassociated X-chromosome. Right: Same as inset except that PolII and Xist signals are presented independently. Far right: Z-stack projections of the cell (upper) and Xist-coated X-chromosome (lower). Scale bar: $5 \mu \mathrm{m}$, Inset: $1 \mu \mathrm{m}$.

c, 3D Amira reconstruction of WT and $\Delta \mathrm{E}$ cells at day 7 of differentiation (optical slices for the same cell are shown in Figure 3E) stained for RNA-polII (green), Xist (red), and DAPI (intensity rendering white/low to orange/high). Inset: Enlargement of the MS2-Xist-expressing Xchromosome. Right: Same as left with the DAPI channel removed.

d, Histogram showing quantification of RNA-PollI exclusion from $X_{i s t}{ }^{\mathrm{MS} 2}$-coated territory in WT and $\Delta \mathrm{E}$ cells at day 3 and 7 of differentiation. 50 cells with $X i s t^{\mathrm{MS} 2}$ clouds were counted from 2 coverslips from 1 differentiation experiment. Significance testing was done using a 2-tailed students t-test (p-values: $*<0.05)$. 


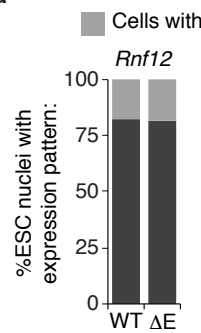

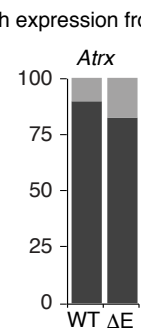
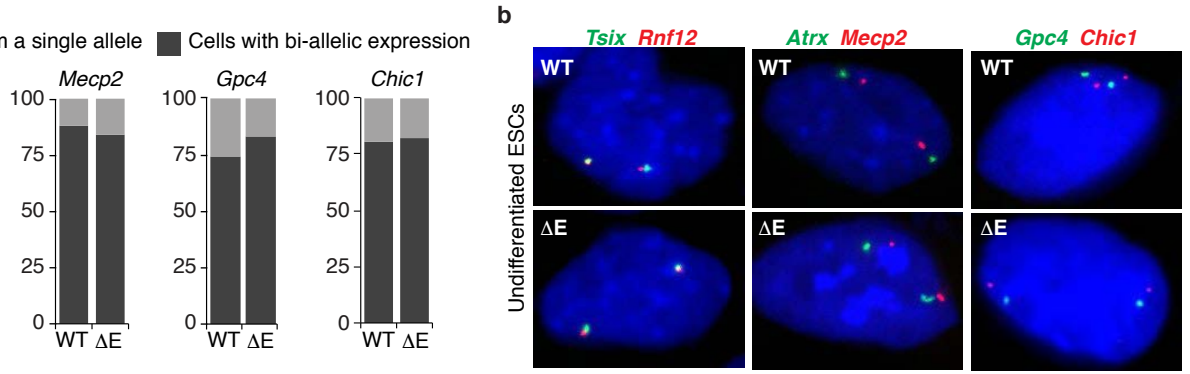

Single spot not co-localizing with Xist $\left.\begin{array}{l}\text { Single spot } \\ \text { co-localizing with Xist } \\ \text { Bi-allelic }\end{array}\right] \begin{aligned} & \text { Lack of } \\ & \text { silencing }\end{aligned}$

Gpc4

Bi-allelic
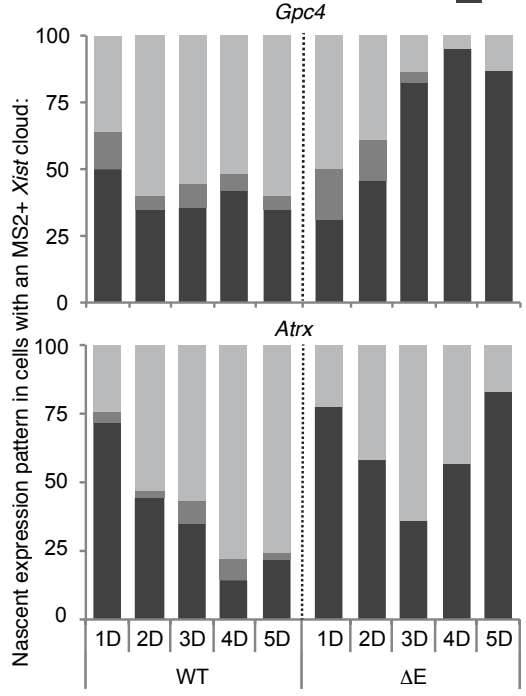

d

Single spot not co-localizing with Xist
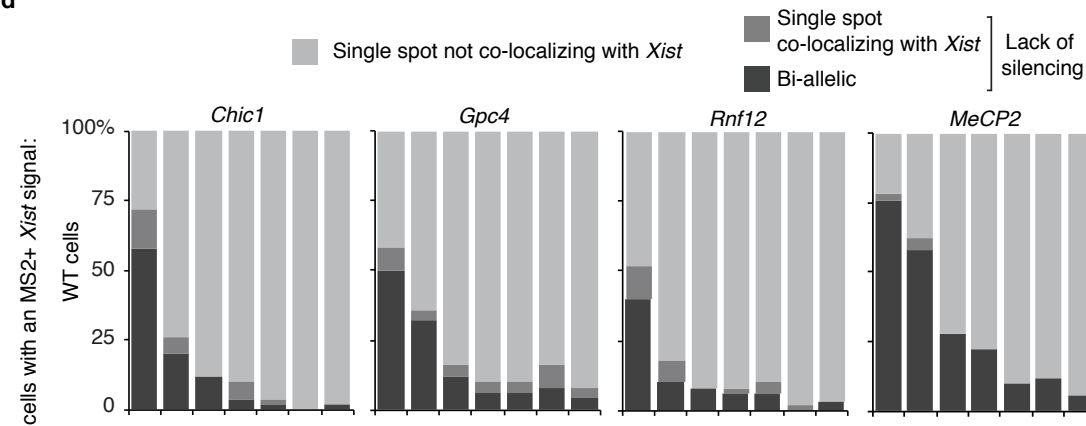

Gpc4

Rnf12
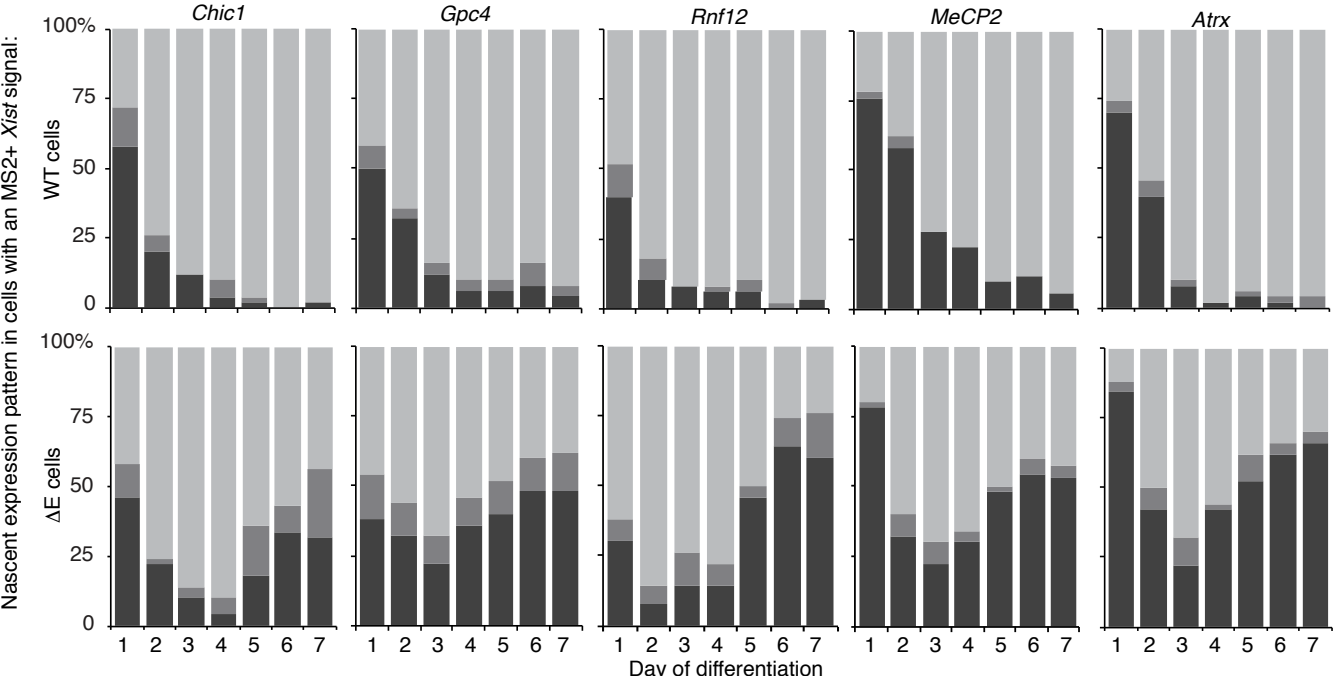


\section{Extended Data Figure 12: Loss of the E-repeat does not interfere with X-linked gene expression in ESCs but prevents continued gene silencing during the initiation of XCI.}

a, Histograms showing the nascent transcription pattern (mono- or bi-allelic) of indicated X-linked genes (Rnf12 (Rlim), Atrx, Mecp2, Gpc4 and Chic1) in undifferentiated female WT and $\Delta \mathrm{E}$ ESCs. 64-67 cells were counted per sample from one experiment.

b, Representative epifluorescent images of cells counted in (a). FISH signals against nascent transcripts are shown in green and red as indicated. Co-localized foci appear yellow. DAPI is shown in blue.

c, Histograms showing the quantification of nascent expression patterns of the X-linked genes Gpc4 and Atrx in WT and $\Delta \mathrm{E}$ cells displaying an $\mathrm{MS} 2+X i s t^{129}$-coated X-chromosome, across 5 days of differentiation. These data are derived from an independent differentiation from that shown in Figure 3c. 50 cells with an MS2+Xist ${ }^{129}$ cloud were counted per time point.

d, Histograms showing the quantification of nascent expression patterns of indicated X-linked genes in WT and $\Delta \mathrm{E}$ cells displaying an MS2+Xist ${ }^{129}$-coated X-chromosome, across 7 days of differentiation. 50 cells with an MS2+Xist ${ }^{129}$ clouds were counted per time point. These data are derived from an independent differentiation from that shown in (c) and Figure 3c.

e, Histogram showing the quantification of nascent expression patterns of the X-linked gene Tsix in $\mathrm{WT}$ and $\Delta \mathrm{E}$ cells across 5 days of differentiation. Note that these data were not scored relative to $X_{i s t}{ }^{\mathrm{MS} 2}$ expression (ie are derived from cells expressing either the 129 or cas allele of Xist). 77167 cells were counted per time point, except for the $\Delta \mathrm{E}$ cells at day 5 , for which only 47 cells were counted. 
bioRxiv preprint doi: https://doi.org/10.1101/2020.03.09.979369; this version posted March 12, 2020. The copyright holder for this preprint (which was not certified by peer review) is the author/funder. All rights reserved. No reuse allowed without permission.
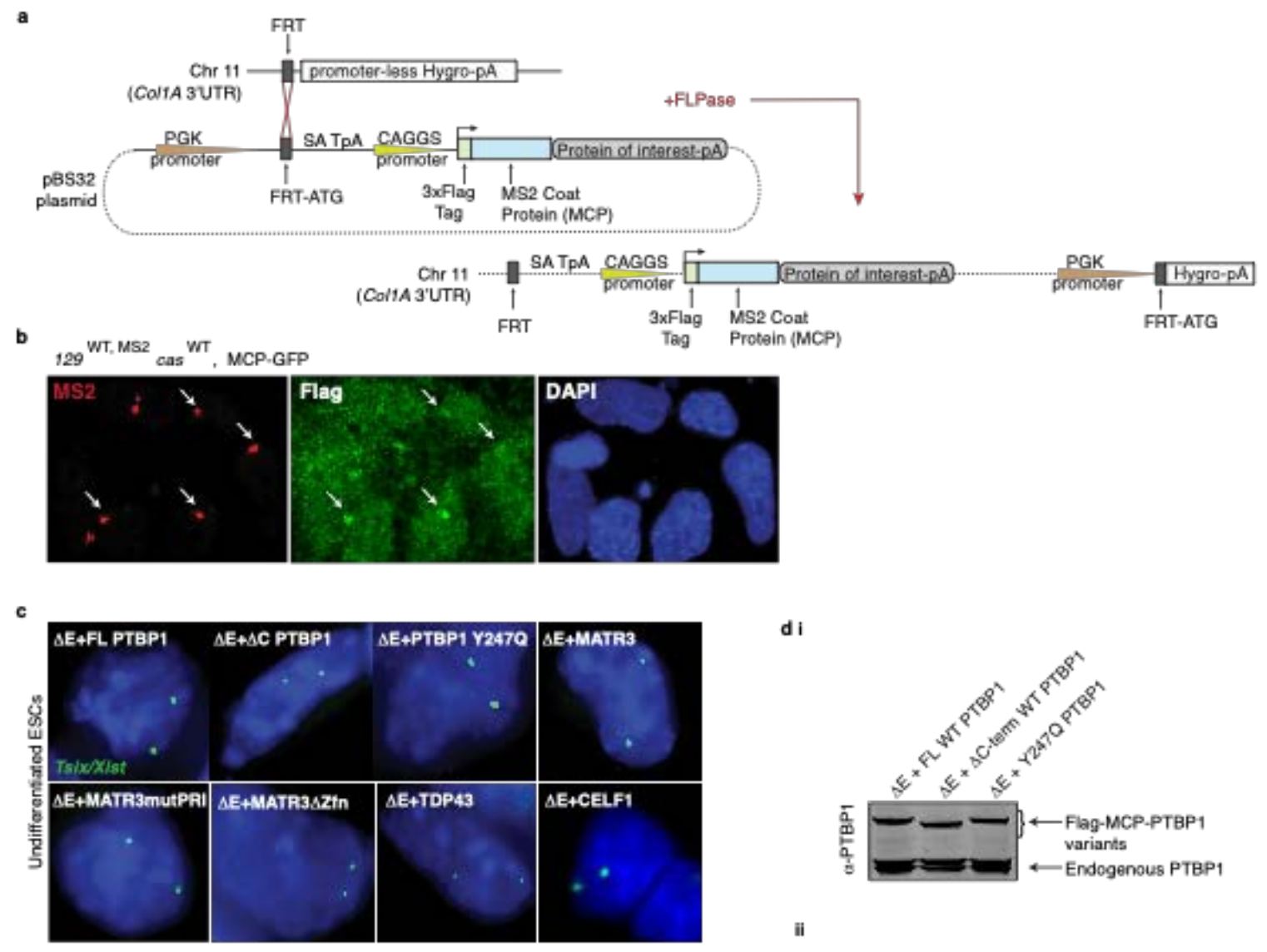

di
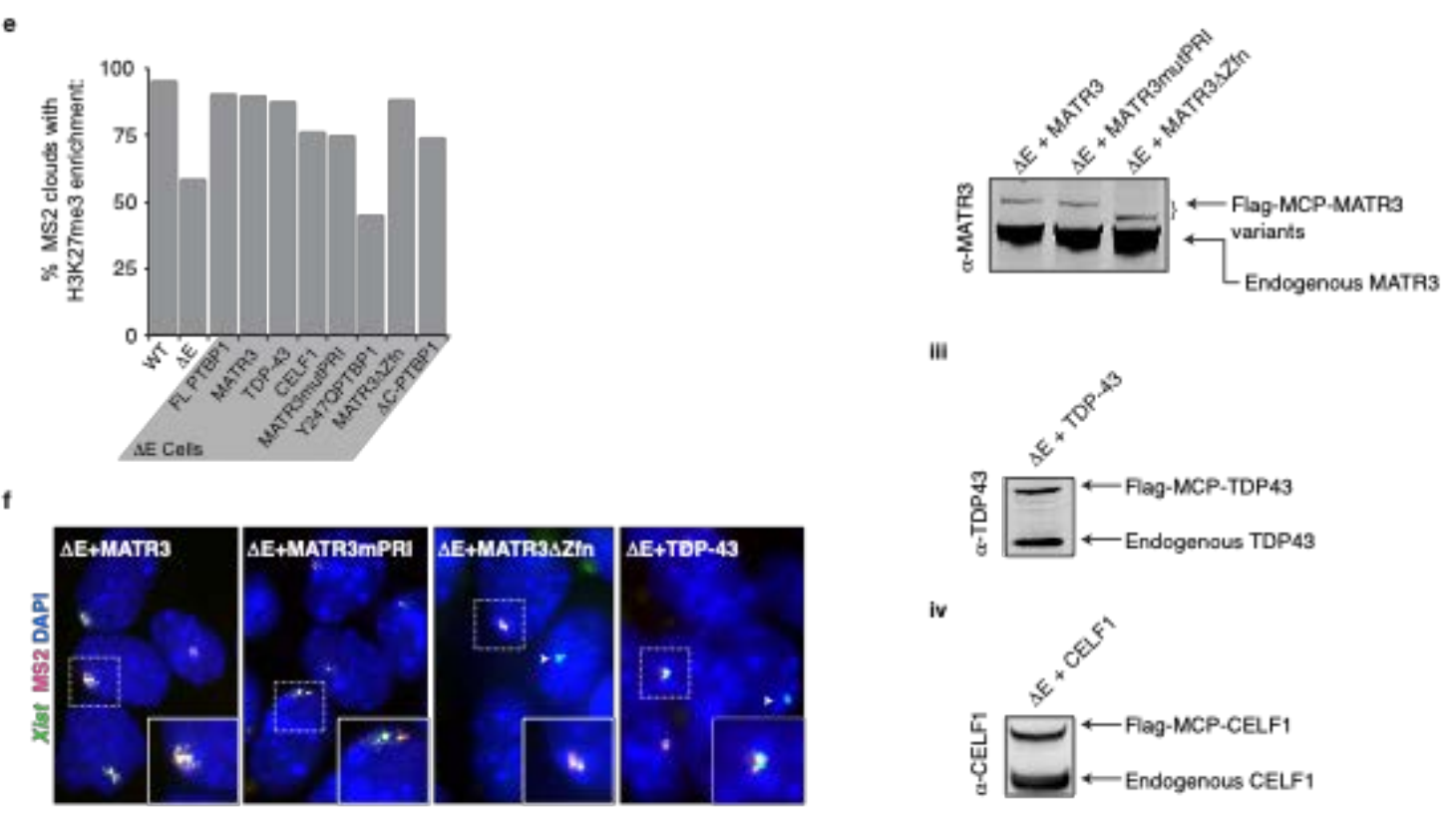

III

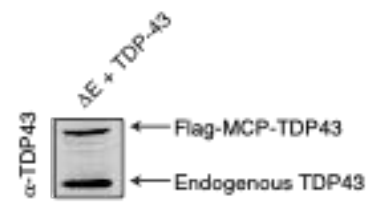

iv
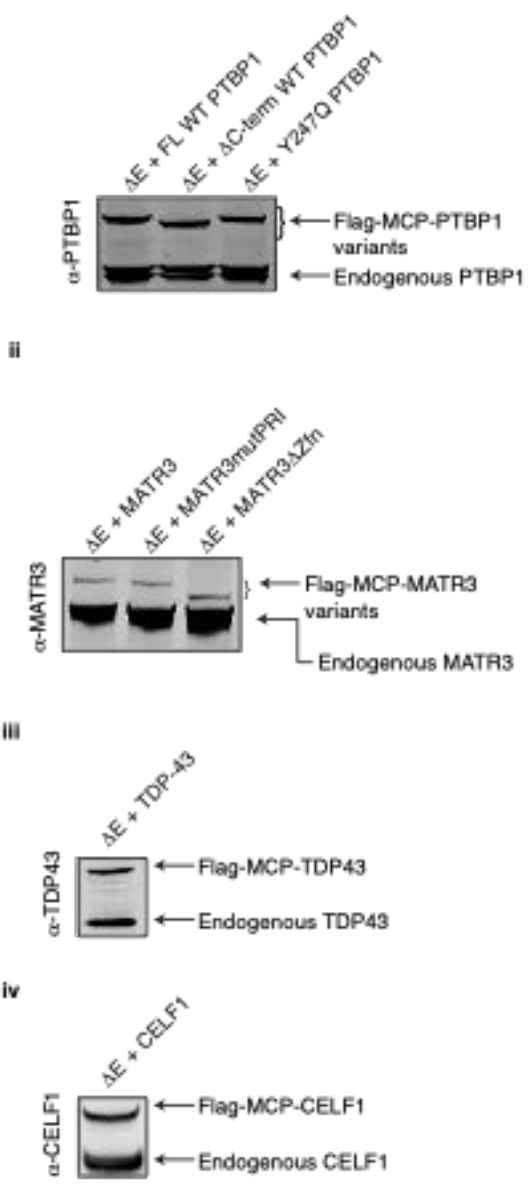


\section{Extended Data Figure 13: A site-specific recombination-based approach to rescue phenotypes associated with loss of the E-repeat via expression of MCP-fusions of E-repeat binding proteins}

a, Diagram illustrating the Flp-In approach taken to constitutively express the MCP fusion proteins in WT and $\triangle \mathrm{E}$ cells (see methods). Note that only the MCP-GFP fusion protein was expressed in WT cells.

b, MCP-GFP fusion protein recruitment to MS2+Xist in WT cells at day 7 of differentiation is shown with representative epifluorescent images. Cells were CSK-extracted (see methods) prior to immunostaining for the Flag epitope (green) and RNA FISH against MS2 (red). DAPI staining is shown in blue. Arrows indicate MS2+Xist ${ }^{129}$ clouds along with co-localizing Flag-MCP-GFP enrichment.

c, RNA FISH images of Tsix transcripts (using a DNA probe detecting both Xist (which is not expressed in undifferentiated ESCs) and its antisense transcript $T$ six) in undifferentiated $\Delta \mathrm{E}$ ESCs expressing the indicated MCP fusion proteins as illustrated in Figure 4b. Tsix signal is in green, DAPI in blue. 2 Tsix spots were used as a proxy to confirm presence of 2 X-chromosomes in the targeted ESC lines.

d i, Immunoblot against PTBP1 using lysates from undifferentiated $\triangle \mathrm{E}$ ESCs expressing fulllength PTBP1 or PTBP1 mutants fused to MCP. ii, Immunoblot against MATR3 using lysates from undifferentiated $\triangle \mathrm{E}$ ESCs expressing full-length MATR3 and MATR3 mutants fused to MCP. iii, Immunoblot against TDP-43 using lysates from the undifferentiated $\triangle \mathrm{E}$ ESCs expressing full-length TDP-43 fused to MCP. iv, Immunoblot against CELF1 using lysates from the undifferentiated $\triangle \mathrm{E}$ ESCs expressing full-length CELF1 fused to MCP.

e, Histogram showing the percentage of MS2+Xist ${ }^{129}$ clouds that also show enrichment of $\mathrm{H} 3 \mathrm{~K} 27 \mathrm{me} 3$ in $\mathrm{WT}$ or $\Delta \mathrm{E}$ cells, or $\Delta \mathrm{E}$ cells expressing the indicated MCP-fusion protein, differentiated for 7 days. 83-103 cells were counted per line, from one experiment.

f, Representative epifluorescence images of RNA FISH against Xist (green) and MS2 (red) in day 7 differentiated $\Delta \mathrm{E}$ cell lines expressing the indicated variants of Flag-tagged MCP fusion proteins. Inset: enlargement of MS2+Xist ${ }^{129}$ cloud outlined with hatched lines. Chosen clouds are representative of the $\mathrm{MS} 2+X_{i s t}{ }^{129}$ cloud phenotype in $\Delta \mathrm{E}$ cells upon rescue with the indicated protein variant. Arrowheads indicate WT Xist clouds in $\Delta \mathrm{E}$ cells, derived from the cas allele. 
bioRxiv preprint doi: https://doi.org/10.1101/2020.03.09.979369; this version posted March 12, 2020. The copyright holder for this preprint (which was not certified by peer review) is the author/funder. All rights reserved. No reuse allowed without permission.

a

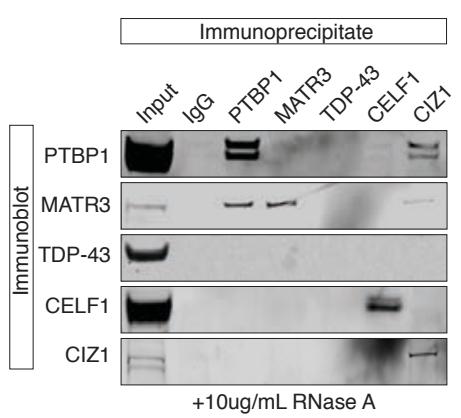




\section{Extended Data Figure 14: PTBP1 and MATR3 interact in the absence of RNA}

Immunoprecipitation of PTBP1, MATR3, CELF1, TDP-43 and CIZ1 from ESC extracts (RNase treated) and detection of co-precipitated proteins with the same antibodies by immunoblotting (to go with Figure 4f, where no RNAse treatment was performed). 
bioRxiv preprint doi: https://doi.org/10.1101/2020.03.09.979369; this version posted March 12, 2020. The copyright holder for this preprint (which was not certified by peer review) is the author/funder. All rights reserved. No reuse allowed without permission.

a

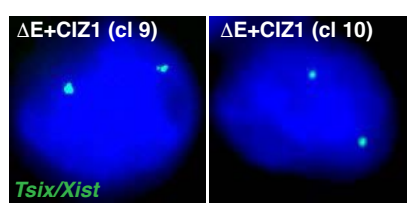

b

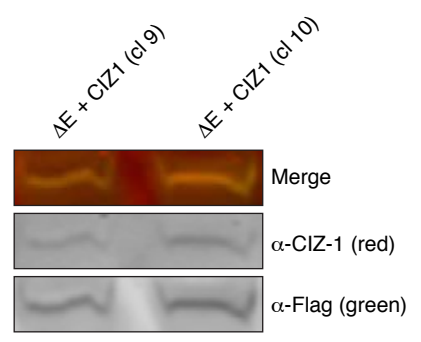

c

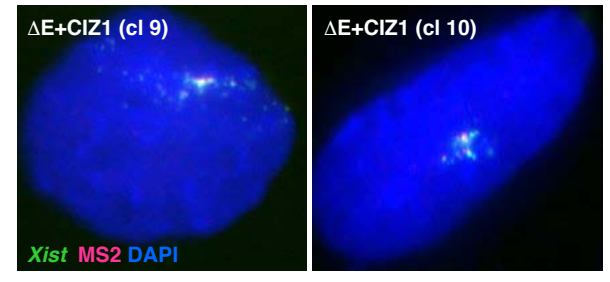

d

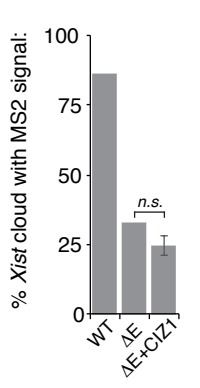

e

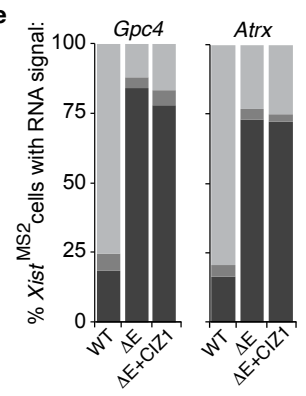




\section{Extended Data Figure 15: Expression of a Flag-MCP-CIZ1 fusion protein does not rescue phenotypes due to loss of the E-repeat}

a, Representative RNA FISH images showing biallelic Tsix expression in MCP-CIZ1 expressing undifferentiated $\Delta \mathrm{E}$ ESCs. Tsix FISH signal is in green, DAPI in blue. 2 Tsix spots were used as a proxy to confirm presence of $2 \mathrm{X}$-chromosomes. Two $\Delta \mathrm{E}$ clones expressing the CIZ1 fusion protein (9 and 10) are shown.

b, Immunoblot against CIZ1 and Flag using lysates from undifferentiated MCP-CIZ1 expressing $\triangle \mathrm{E}$ ESCs.

c, Representative epifluorescence images of RNA FISH s against Xist (green) and MS2 (red) in day 7 differentiated MCP-CIZ1 expressing $\triangle \mathrm{E}$ cells. Two independent clones are shown.

d, Histogram showing the number of nuclei with an Xist cloud that also displayed a co-localizing MS2 signal, as detected by RNA FISH, in WT, $\triangle \mathrm{E}$, or $\Delta \mathrm{E}$ cells expressing Flag-tagged MCPCIZ1. Error bar is derived from the analysis of two CIZ1 rescue clones ( 9 and 10) $120-189$ cells were counted per sample from one experiment. There was no significant difference between the $\Delta \mathrm{E}$ cells and $\triangle \mathrm{E}$ cells MCP-CIZ1 rescue lines (2-tailed students t-test).

e, Histograms showing the quantification of nascent Gpc4 or Atrx nascent expression patterns in $\mathrm{WT}, \Delta \mathrm{E}$, or $\Delta \mathrm{E}$ cells expressing Flag-tagged MCP-CIZ1 (clone 9) displaying MS2+Xist ${ }^{129}$ expression at differentiation day 7 as described in (d). 50 cells with an Xist-MS2 cloud/FISH signal were counted per sample. 
bioRxiv preprint doi: https://doi.org/10.1101/2020.03.09.979369; this version posted March 12, 2020. The copyright holder for this preprint (which was not certified by peer review) is the author/funder. All rights reserved. No reuse allowed without permission.

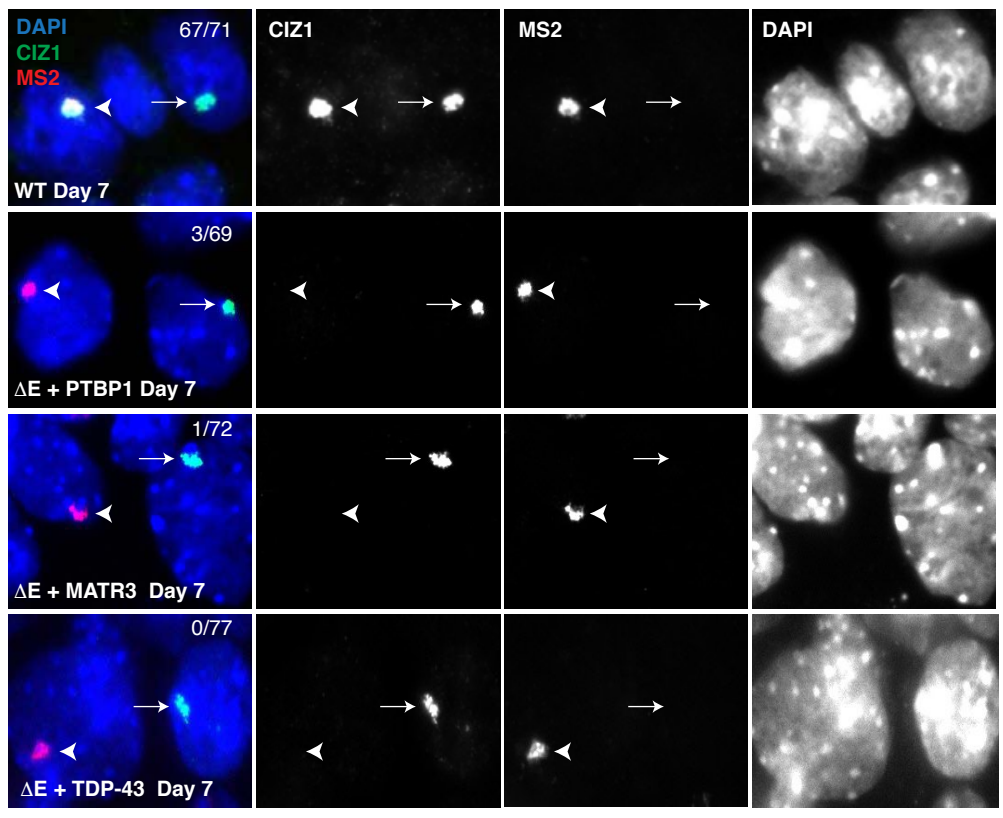




\section{Extended Data Figure 16: CIZ1 does not enrich on the Xi upon rescue of the E-repeat phenotypes with MCP-PTBP1, -MATR3 or -TDP-43}

Representative fluorescence images showing the Xi-enrichment of CIZ1 (immunostaining, green) on the MS2+Xist ${ }^{129}$ chromosome (RNA FISH, red) in WT cells at 7 days of differentiation. DAPI stained nuclei are shown in blue. The same staining for $\Delta \mathrm{E}$ cells expressing MS2-CP-PTBP1, MS2-CP-MATR3 or MS2CP-TDP43 at day 7 of differentiation is shown below and reveals that CIZ1 does not accumulate in these cell lines. The CIZ1 Xi-enrichment seen in WT and $\Delta \mathrm{E}$ cells expressing MCP-PTBP1, MCP-MATR3 or MCP-TDP43 that does not co-localize with the MS2+ signal derives from the Cas Xi that expresses a wild-type Xist (indicated by arrow). Arrowhead indicates rescued cloud derived from the $\Delta \mathrm{E} 129$ allele. Numbers at the top right in the merged image represent the percentage of MS2 $+X_{i s t}{ }^{129}$ clouds showing CIZ1 co-localization. 100 cells with an $X i s t^{\mathrm{MS} 2}$ cloud were counted per sample, from a single differentiation experiment. 
a

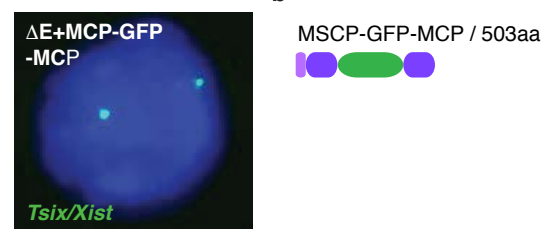

d

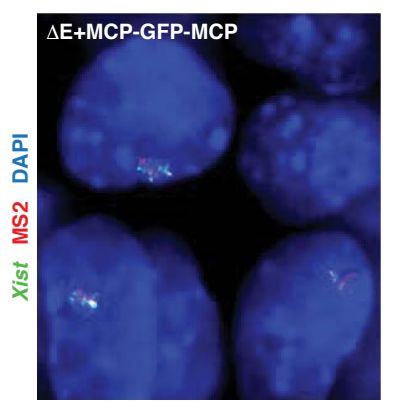

e

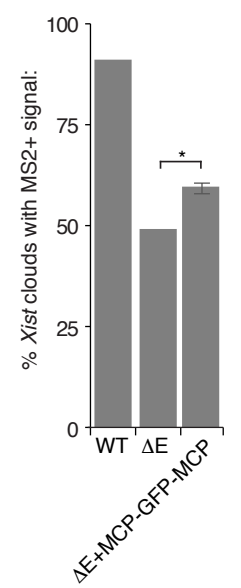

f

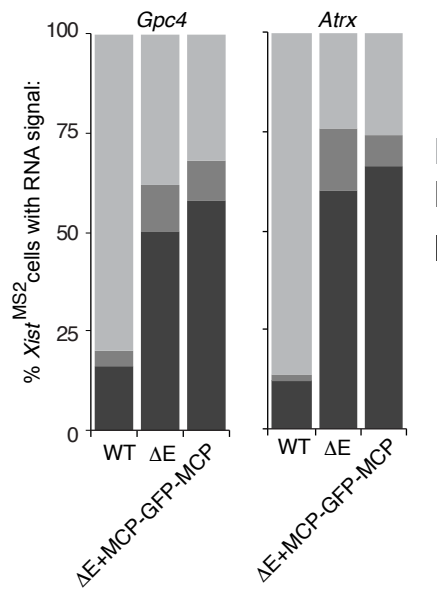

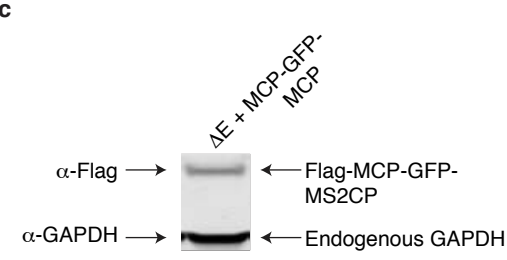

Single spot not co-localizing with Xist

Single spot

co-localizing with Xist Lack of

Bi-allelic silencing 


\section{Extended Data Figure 17: Expression of a Flag-tagged MCP-GFP-MCP fusion protein does not rescue the phenotypes associated with E-repeat loss}

a, Representative RNA FISH images showing biallelic Tsix expression in MCP-GFP-MCP expressing undifferentiated $\Delta \mathrm{E}$ ESCs. Tsix FISH signal is in green, DAPI in blue. 2 Tsix spots were used as a proxy to confirm presence of $2 \mathrm{X}$-chromosomes.

b, Illustration of Flag-tagged MCP-GFP-MCP fusion protein. See Figure $4 \mathrm{~b}$ for key.

c, Immunoblot against the Flag-tag and GAPDH using lysates from undifferentiated MCP-GFPMCP $\triangle \mathrm{E}$ ESCs.

d, Representative epifluorescence images of Xist (green) and MS2 (red) detected by RNA FISH in day 7 differentiated MCP-GFP-MCP-expressing $\triangle \mathrm{E}$ cells.

e, Histogram showing the percentage of Xist clouds with co-localizing MS2 signal at day 7 of differentiation for WT or $\Delta \mathrm{E}$ cells and $\Delta \mathrm{E}$ cells expressing the Flag-MCP-GFP-MCP fusion protein. 108-144 cells were counted per sample from 2 independent clones from one differentiation experiment. There was no significant difference between the $\Delta \mathrm{E}$ cells and experimental $\Delta \mathrm{E} \mathrm{MCP}-$ GFP-MCP rescue lines (2-tailed students t-test).

f, Histograms showing the quantification of nascent Gpc4 or Atrx nascent expression patterns in cells displaying MS2+ Xist expression at differentiation day 7 in WT or $\Delta \mathrm{E}$ cells and $\Delta \mathrm{E}$ cells expressing the Flag-MCP-GFP-MCP fusion protein. 50 cells were counted per sample from one experiment. 
bioRxiv preprint doi: https://doi.org/10.1101/2020.03.09.979369; this version posted March 12, 2020. The copyright holder for this preprint (which was not certified by peer review) is the author/funder. All rights reserved. No reuse allowed without permission.
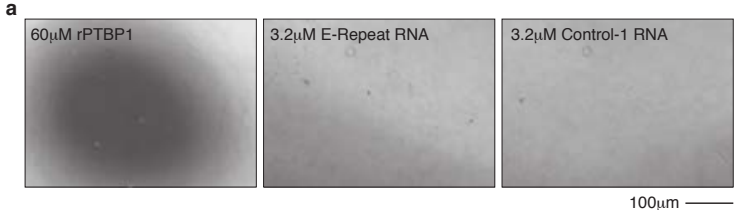

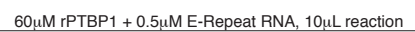
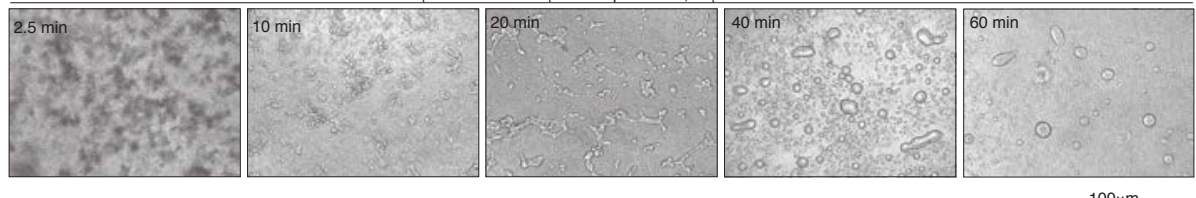

$100 \mu \mathrm{m}$ 


\section{Extended Data Figure 18: Droplet formation by PTBP1 and Xist E-repeat RNA occurs over time}

a, Brightfield images showing the absence of droplet formation with $60 \mu \mathrm{M}$ rPTBP 1 only, $3.2 \mu \mathrm{M}$ E-Repeat RNA only, or 3.2 $\mu \mathrm{M}$ Control RNA only.

b, Brightfield images of droplets formed with $60 \mu \mathrm{M} \mathrm{rPTBP} 1$ and $3.2 \mu \mathrm{M}$ E-repeat RNA taken over a $1 \mathrm{~h}$ time course. 
a

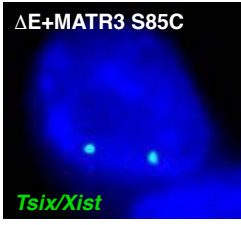

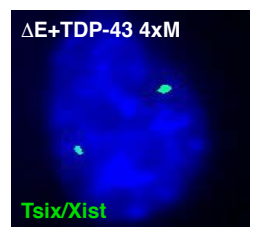

b i

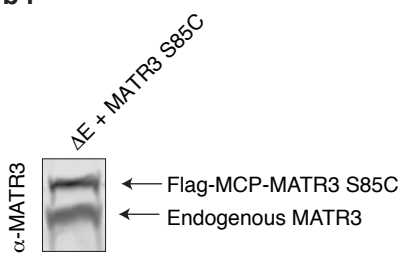

ii

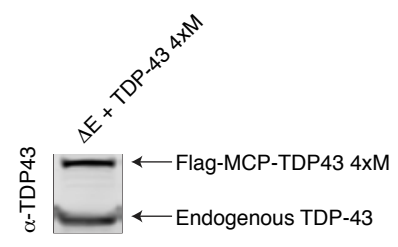

c i
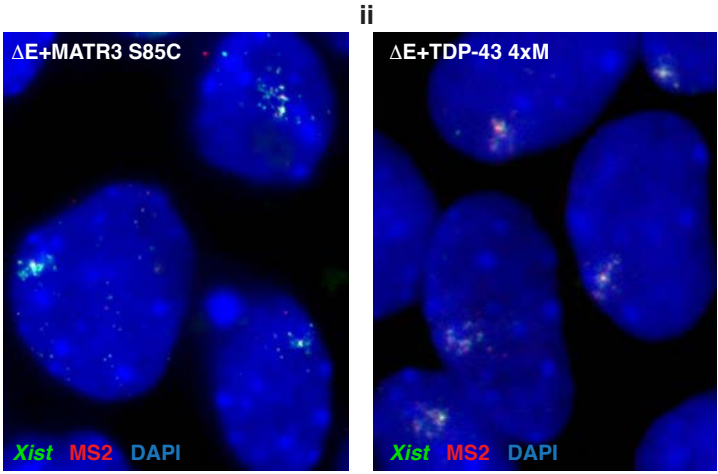


\section{Extended Data Figure 19: Mutations in MATR3 and TDP-43 that prevent self-association of these factors do not rescue the phenotypes observed upon loss of the E-repeat}

a, Representative RNA FISH images showing biallelic Tsix expression in MCP-MATR3-S85C and MCP-TDP-43-4xM expressing undifferentiated $\triangle \mathrm{E}$ ESCs. Tsix FISH signal is in green, DAPI in blue. 2 Tsix spots were used as a proxy to confirm presence of $2 \mathrm{X}$ chromosomes.

b i, Immunoblot against MATR3 using lysates from MCP-MATR3 S85C expressing undifferentiated $\Delta \mathrm{E}$ ESCs. ii, Immunoblot against TDP-43 using lysates from MCP-TDP-43-4xM expressing undifferentiated $\Delta \mathrm{E}$ ESCs.

c, Representative fluorescence images of Xist (green) and MS2 (red) in day 7 differentiated FlagMCP-MATR3-S85C expressing $\triangle \mathrm{E}$ cells (i), and Flag-MCP- TDP-43-4xM expressing $\Delta \mathrm{E}$ cells (ii). 
bioRxiv preprint doi: https://doi.org/10.1101/2020.03.09.979369; this version posted March 12, 2020. The copyright holder for this preprint (which was not certified by peer review) is the author/funder. All rights reserved. No reuse allowed without permission.

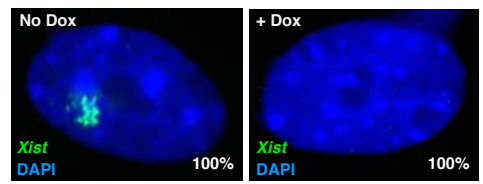




\section{Extended Data Figure 20: Dox treatment effectively deletes Xist in engineered MEFs}

a, Images showing Xist as detected by RNA FISH in MEFs (Xist $t^{2 \operatorname{lox} / 2 \operatorname{lox}}$, Rosa26 ${ }^{\mathrm{M} 2 \mathrm{rtTA} / \mathrm{tetO}-\mathrm{Cre}-}$ recombinase) before or after $96 \mathrm{~h}$ of dox treatment. Numbers indicate percentage of cells with the displayed Xist expression pattern across 50 cells from 2 biological replicates. These data indicate that Xist excision completes in all cells. 


\section{Extended Data Table 1: Primer information}

Primer Useage

Primers used for cloning \& sequencing of the pCR2.1-Puro-Xist $\Delta \mathrm{E}$ targeting plasmid

WRM163 Forward primer for $5^{\prime}$ homology arm in $\triangle \mathrm{E}$ targeting construct for infusion cloning into EcoRV site in PCR2.1-TOPO-Puro GCCAGTGTGATGGATCTAGTAATCAAATTATCATTTGTAG

WRM164 Reverse primer for $5^{\prime}$ homology arm in $\triangle \mathrm{E}$ targeting construct for infusion cloning into EcoRV site in PCR2.1-TOPO-Puro GCGAATTCTGCAGATTAGACACACCCACAATACACAC

WRM165 Forward primer for 3' homology arm in $\triangle \mathrm{E}$ targeting construct for infusion cloning into EcoRV site in PCR2.1-TOPO-Puro CGTTACTAGTGGATCTACATCATTCCGTCCGGTCAGG

WRM166 Reverse primer for 3' homology arm in $\triangle \mathrm{E}$ targeting construct for infusion cloning into EcoRV site in PCR2.1-TOPO-Puro TACCGAGCTCGGATCTTGCCAATGTGCTATGAGCATG

M13F Sequencing primer for 5'HA within the PCR2.1-TOPO-Puro Plasmid E repeat deletion targeting plasmid TGTAAAACGACGGCCAGT

WRM136 Sequencing primer for 5'HA within the PCR2.1-TOPO-Puro Plasmid E repeat deletion targeting plasmid GGAATTTTCAACAATTTTTGTAG

WRM137 Sequencing primer for 5'HA within the PCR2.1-TOPO-Puro Plasmid E repeat deletion targeting plasmid TCTCTGAATATCCTCAAACTCAG

WRM138 Sequencing primer for 5'HA within the PCR2.1-TOPO-Puro Plasmid E repeat deletion targeting plasmid TAGGTGGAGATGGGGCATGAGG

WRM139 Sequencing primer for 5'HA within the PCR2.1-TOPO-Puro Plasmid E repeat deletion targeting plasmid AGAGCACCAGATCTCATTACAG

WRM140 Sequencing primer for 5'HA within the PCR2.1-TOPO-Puro Plasmid E repeat deletion targeting plasmid TGTGAGGCAGGGCCATGTACACG

WRM141 Sequencing primer for 5'HA within the PCR2.1-TOPO-Puro Plasmid E repeat deletion targeting plasmid AAATATGCCCTGGAGTTGCATG

APJ304 Sequencing primer for Puromycin Cassette within the PCR2.1-TOPO-Puro $\triangle E$ plasmid

GACAAATGGAAGTAGCACGTCTCAC

APJ305 Sequencing primer for Puromycin Cassette within the PCR2.1-TOPO-Puro $\triangle E$ plasmid CACGCCGGAGAGCGTCGAAGCGG

APJ306 Sequencing primer for Puromycin Cassette within the PCR2.1-TOPO-Puro $\triangle E$ plasmid TGCCTTCCTTGACCCTGGAAGGT

APJ307 Sequencing primer for 3'HA within the PCR2.1-TOPO-Puro Plasmid E repeat deletion targeting plasmid TACATCATTCCGTCCGGTCAGGA

WRM147 Sequencing primer for 3'HA within the PCR2.1-TOPO-Puro Plasmid E repeat deletion targeting plasmid TAGCTGGATAGTATTGTGCAAG

WRM148 Sequencing primer for 3'HA within the PCR2.1-TOPO-Puro Plasmid E repeat deletion targeting plasmid TTAATGATGAGAGACTCTAAGG 


\section{Extended Data Table 1: Primer information (continued)}

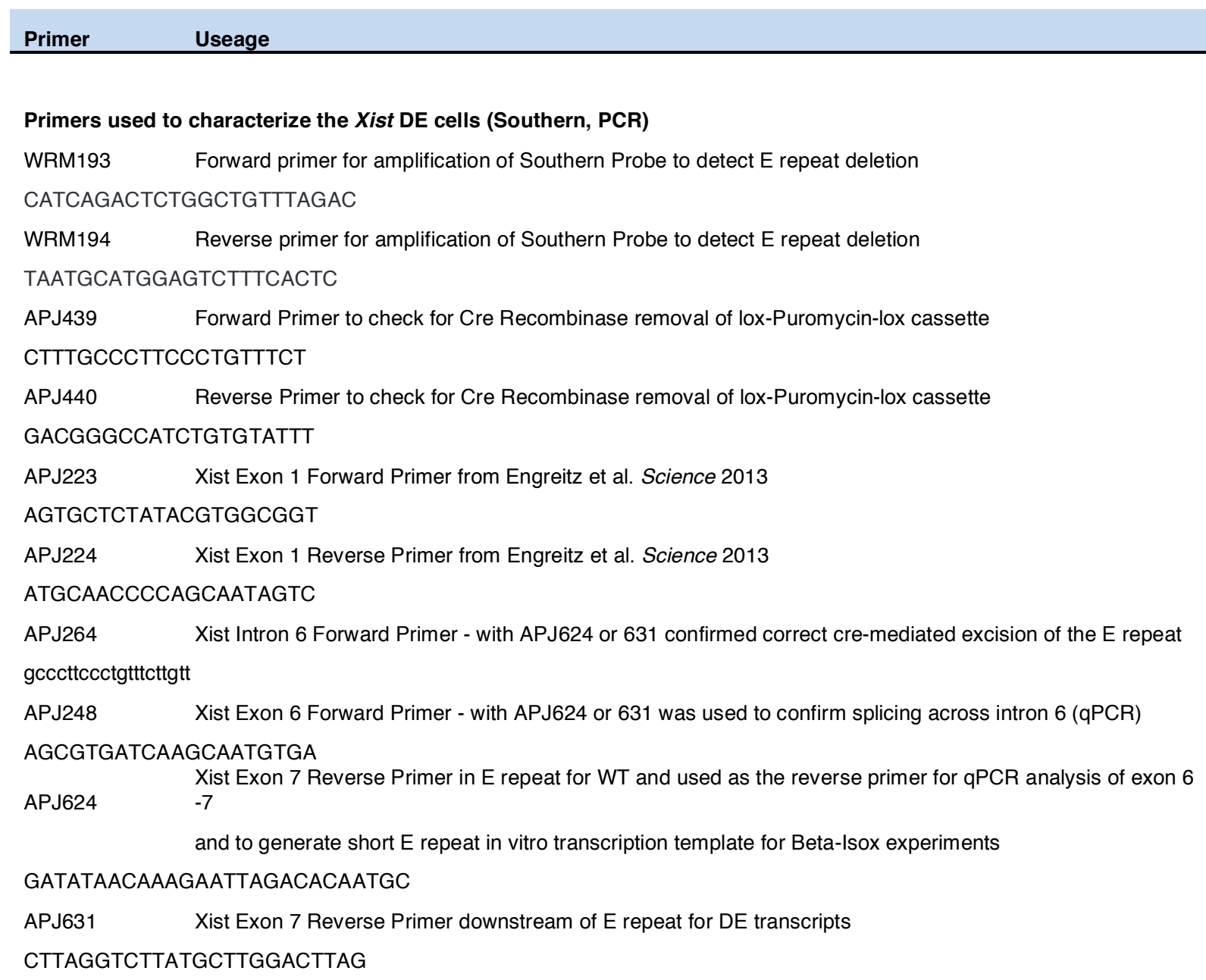

\section{Primers used to clone \& sequence the pBS32-FRT parent and fusion protein expression plasmids}

APJ526 Forward Infusion primer for Flag-MS2CP to make the MS2CP-Nhe1 linker-IRES-Puro pBS32 plasmid

tttggcaaaGAATTGCCACCatggactacaaagaccatgacggtgattataaagatcatgatatcgattacaaggatgacgatgacggtgga

ATGGCTTCTAACTTTACTCAGTTCG

APJ570 Reverse Infusion primer for MS2CP to make the MS2CP-Nhe1 linker-IRES-Puro pBS32 plasmid CTTCGGCCAGTAACGtccGCTAGCccGTAGATGCCGGAGTTTGCTGCG

APJ528 Forward infusion primer for IRES to make the MS2CP-Nhe1 linker-IRES-Puro pBS32 plasmid CGTTACTGGCCGAAGCCGCTTGG

APJ571 Reverse infusion primer for IRES to make the MS2CP-Nhe1 linker-IRES-Puro pBS32 plasmid

ATTATCATCGTGTTTTTCAAAGG

Forward infusion primer for Puromycin cassette to make the MS2CP-Nhe1 linker-IRES-Puro pBS32
plasmid

AAACACGATGATAATATGACCGAGTACAAGCCCACGGTGC 


\section{Extended Data Table 1: Primer information (continued)}

\begin{tabular}{|c|c|}
\hline Primer & Useage \\
\hline APJ533 & Reverse infusion primer for puromycin cassette to make the MS2CP-Nhe1 linker-IRES-Puro pBS32 plasmid \\
\hline \multicolumn{2}{|c|}{ cctgaggagtGAATTTCAGGCACCGGGCTTGCGGGTCATG } \\
\hline APJ573 & Forward infusion primer for eGFP into Nhe1 cut site within MS2CP-Nhe1 linker-IRES-Puro pBS32 plasmid \\
\hline \multicolumn{2}{|c|}{ GCATCTACggGCTAGggATGGTGAGCAAGGGCGAGGAG } \\
\hline APJ574 & Reverse infusion primer for eGFP into Nhe1 cut site within MS2CP-Nhe1 linker-IRES-Puro pBS32 plasmid \\
\hline \multicolumn{2}{|c|}{ AGTAACGtccGCTAGTTACTTGTACAGCTCGTCCATG } \\
\hline APJ575 & Forward infusion primer for WT Matrin3 into Nhe1 cut site within MS2CP-Nhe1 linker-IRES-Puro pBS32 plasmid \\
\hline \multicolumn{2}{|c|}{ GCATCTACggGCTAGggATGTCCAAGTCATTCCAGCAGTC } \\
\hline APJ576 & Reverse infusion primer for WT Matrin3 into Nhe1 cut site within MS2CP-Nhe1 linker-IRES-Puro pBS32 plasmid \\
\hline \multicolumn{2}{|c|}{ AGTAACGtccGCTAGTTAAGTTTCCTTCTTCTGTCTGC } \\
\hline APJ575 & Forward infusion primer for $5^{\prime}$ fragment of mutPRI Matrin3 into Nhe1 site (2 piece infusion ) within pBS32-MS2CP-Nhe1 \\
\hline \multicolumn{2}{|c|}{ GCATCTACggGCTAGggATGTCCAAGTCATTCCAGCAGTC } \\
\hline APJ586 & Reverse infusion primer for $5^{\prime}$ fragment of mutPRI Matrin3 into Nhe1 site (2 piece infusion ) within pBS32-MS2CP-Nhe1 \\
\hline \multicolumn{2}{|c|}{ TGGTGCTGGATTTGTAGACTGCTG } \\
\hline APJ585 & Forward infusion primer for 3 ' fragment of mutPRI Matrin3 into Nhe1 site (2 piece infusion ) within pBS32-MS2CP-Nhe1 \\
\hline \multicolumn{2}{|c|}{ ACAAATCCAGCACCAGGAGCCGCCGCCCCTCCAGCCCCCTCATTTCATCTTGGGGGACCAGC } \\
\hline APJ576 & Reverse infusion primer for $3^{\prime}$ fragment of mutPRI Matrin3 into Nhe1 site (2 piece infusion ) within pBS32-MS2CP-Nhe1 \\
\hline \multicolumn{2}{|c|}{ AGTAACGtccGCTAGTTAAGTTTCCTTCTTCTGTCTGC } \\
\hline APJ577 & Forward infusion primer for PTBP1 into Nhe1 cut site (for 1 piece infusion insertion) within pBS32-MS2CP-Nhe1 \\
\hline \multicolumn{2}{|c|}{ GCATCTACggGCTAGggATGGACGGCATTGTCCCAGATATAG } \\
\hline APJ578 & Reverse infusion primer for PTBP1 into Nhe1 cut site (for1 piece infusion insertion) within pBS32-MS2CP-Nhe1 \\
\hline \multicolumn{2}{|c|}{ AGTAACGtccGCTAGTTAGATGGTGGACTTGGAGAAGG } \\
\hline APJ577 & Forward infusion primer for $5^{\prime}$ fragment of WT PTBP1 into Nhe1 site (2 piece infusion) within pBS32-MS2CP-Nhe1 \\
\hline \multicolumn{2}{|c|}{ GCATCTACggGCTAGggATGGACGGCATTGTCCCAGATATAG } \\
\hline APJ609 & Reverse infusion primer for $5^{\prime}$ fragment of WT PTBP1 into Nhe1 site (2 piece infusion) within pBS32-MS2CP-Nhe1 \\
\hline \multicolumn{2}{|c|}{ AGCGTGCAGCAGGCGTTGTAGATGTTCTGCCCGTCCAGCG } \\
\hline APJ581 & Forward infusion primer for $3^{\prime}$ fragment of WT PTBP1 into Nhe1 site (2 piece infusion) within pBS32-MS2CP-Nhe1 \\
\hline \multicolumn{2}{|c|}{ CGCCTGCTGCACGCTGCGCATCGAC } \\
\hline APJ578 & Reverse infusion primer for $3^{\prime}$ fragment of WT PTBP1 into Nhe1 site (2 piece infusion) within pBS32-MS2CP-Nhe1 \\
\hline \multicolumn{2}{|c|}{ AGTAACGtccGCTAGTTAGATGGTGGACTTGGAGAAGG } \\
\hline APJ577 & Forward infusion primer for 5 ' fragment of Y247Q PTBP1 into Nhe1 site (2 piece infusion) within pBS32-MS2CP-Nhe1 \\
\hline \multicolumn{2}{|c|}{ GCATCTACggGCTAGggATGGACGGCATTGTCCCAGATATAG } \\
\hline APJ582 & Forward infusion primer for 5' fragment of Y247Q PTBP1 into Nhe1 site (2 piece infusion) within pBS32-MS2CP-Nhe1 \\
\hline \multicolumn{2}{|c|}{ AGCGTGCAGCAGGCGTTCtgGATGTTCTGCCCGTCCAGCG } \\
\hline APJ581 & Forward infusion primer for 3 ' fragment of Y247Q PTBP1 into Nhe1 site (2 piece infusion) within pBS32-MS2CP-Nhe1 \\
\hline
\end{tabular}




\section{Extended Data Table 1: Primer information (continued)}

\begin{tabular}{|c|c|}
\hline APJ578 & Reverse infusion primer for 3' fragment of Y247Q PTBP1 into Nhe1 site (2 piece infusion) within pBS32-MS2CP-Nhe1 \\
\hline \multicolumn{2}{|c|}{ AGTAACGtccGCTAGTTAGATGGTGGACTTGGAGAAGG } \\
\hline APJ636 & TDP43 Coding Sequence forward primer for amplicification from cDNA \\
\hline \multicolumn{2}{|c|}{ ATGTCTGAATATATTCGGGTAACAG } \\
\hline APJ638 & Forward infusion primer for TDP43 coding fragment into Nhe1 cut site within MS2CP-Nhe1 linker-IRES-Puro pBS32 plasmid \\
\hline \multicolumn{2}{|c|}{ GCATCTACggGCTAGggATGTCTGAATATATTCGGGTAACAG } \\
\hline APJ639 & Reverse infusion primer for TDP43 coding fragment into Nhe1 cut site within MS2CP-Nhe1 linker-IRES-Puro pBS32 plasmid \\
\hline \multicolumn{2}{|c|}{ AGTAACGtccGCTAGTTACATTCCCCAGCCAGAAGACTTAG } \\
\hline \multicolumn{2}{|c|}{ cagggtgggtttggtaacagtagag } \\
\hline APJ640 & Reverse primer within MATR3 to generate fragment lacking Zinc Finger 1 - used with APJ575 \\
\hline \multicolumn{2}{|c|}{ ATGGAAGTCTTCAATATTGCTACTTGG } \\
\hline APJ641 & Forward primer within MATR3 to generate fragment lacking Zinc Finger 2 - used with APJ642remove Zinc Finger 1 \\
\hline \multicolumn{2}{|c|}{ CTTGAAATCTACCCAGAATGGAATCC } \\
\hline APJ642 & Reverse primer within MATR3 to generate fragment lacking Zinc Finger 2 - used with APJ641 \\
\hline \multicolumn{2}{|c|}{ TATACCAACAGGAACATTGGGCTG } \\
\hline APJ643 & MATR3 reverse with infusion overhang to join the two DZfn MATR3 fragments together \\
\hline APJ652 & ClZ1 reverse with Nhe1 Infusion overhang \\
\hline \multicolumn{2}{|c|}{ AGTAACGtccGCTAGTCAGGTTTTGaggegtgttgagcgcc } \\
\hline \multicolumn{2}{|c|}{ tagagcctctgctaaccatgttc } \\
\hline ctcagtggtatttgtgagccag & $\begin{array}{l}\text { Sequencing primers for MS2CP-Nhe1 linker-IRES-Puro plasmid } \\
\text { yccag }\end{array}$ \\
\hline APJ525 & Sequencing primers for MS2CP-Nhe1 linker-IRES-Puro plasmid \\
\hline \multicolumn{2}{|c|}{ ATGGCTTCTAACTTTACTCAGTTCG } \\
\hline APJ528 & Sequencing primers for MS2CP-Nhe1 linker-IRES-Puro plasmid \\
\hline
\end{tabular}




\section{Extended Data Table 1: Primer information (continued)}

\begin{tabular}{|c|c|}
\hline Primer & Useage \\
\hline \multicolumn{2}{|c|}{ CGTTACTGGCCGAAGCCGCTTGG } \\
\hline APJ530 & Sequencing primers for MS2CP-Nhe1 linker-IRES-Puro plasmid \\
\hline \multicolumn{2}{|c|}{ ATGACCGAGTACAAGCCCACGGTGC } \\
\hline APJ606 & Sequencing primers for MS2CP-Nhe1 linker-IRES-Puro plasmid \\
\hline \multicolumn{2}{|c|}{ GCCTCTGCGGCCAAAAGCCACGTG } \\
\hline APJ607 & Sequencing primers for MS2CP-Nhe1 linker-IRES-Puro plasmid \\
\hline \multicolumn{2}{|c|}{ GTCACCGAGCTGCAAGAACT } \\
\hline APJ608 & Sequencing primers for MS2CP-Nhe1 linker-IRES-Puro plasmid \\
\hline \multicolumn{2}{|c|}{ CACCAGGGCAAGGGTCTG } \\
\hline APJ612 & Sequencing primers for MS2CP-Nhe1 linker-IRES-Puro plasmid \\
\hline \multicolumn{2}{|c|}{ GTGTATAAGATACACCTGCAAAGG } \\
\hline APJ595 & Sequencing primers for MS2CP-Nhe1 linker-IRES-Puro plasmid with PTBP1 WT and/or Mutant inserted \\
\hline \multicolumn{2}{|c|}{ CCGTGGACGCAGGGATGGCGATGG } \\
\hline APJ596 & Sequencing primers for MS2CP-Nhe1 linker-IRES-Puro plasmid with PTBP1 WT and/or Mutant inserted \\
\hline \multicolumn{2}{|c|}{ CTGGCCCCCCTGGCCATCCCCTcg } \\
\hline APJ597 & Sequencing primers for MS2CP-Nhe1 linker-IRES-Puro plasmid with PTBP1 WT and/or Mutant inserted \\
\hline \multicolumn{2}{|c|}{ CAGTCTCCGAGGAGGATCTCAAGG } \\
\hline APJ598 & Sequencing primers for MS2CP-Nhe1 linker-IRES-Puro plasmid with Matrin3 WT and/or Mutants inserted \\
\hline \multicolumn{2}{|c|}{ GGAGCCACCATACAGAGTACC } \\
\hline APJ599 & Sequencing primers for MS2CP-Nhe1 linker-IRES-Puro plasmid with Matrin3 WT and/or Mutants inserted \\
\hline \multicolumn{2}{|c|}{ CAGGACACACAATGGGTGATCC } \\
\hline APJ600 & Sequencing primers for MS2CP-Nhe1 linker-IRES-Puro plasmid with Matrin3 WT and/or Mutants inserted \\
\hline \multicolumn{2}{|c|}{ CTCAGCAATTTGCCGCATTCTGGC } \\
\hline APJ601 & Sequencing primers for MS2CP-Nhe1 linker-IRES-Puro plasmid with Matrin3 WT and/or Mutants inserted \\
\hline \multicolumn{2}{|c|}{ GTGGGAGACGAGACCGATCTTGC } \\
\hline APJ602 & Sequencing primers for MS2CP-Nhe1 linker-IRES-Puro plasmid with Celf-1 inserted \\
\hline \multicolumn{2}{|c|}{ GCACCAAGCACAGACCATGGAGG } \\
\hline APJ603 & Sequencing primers for MS2CP-Nhe1 linker-IRES-Puro plasmid with Celf-1 inserted \\
\hline \multicolumn{2}{|c|}{ ATGGTGGCCTGGGCAGCAGTGG } \\
\hline APJ604 & Sequencing primers for MS2CP-Nhe1 linker-IRES-Puro plasmid with eGFP inserted \\
\hline \multicolumn{2}{|c|}{ ATGGTGAGCAAGGGCGAGGAG } \\
\hline APJ605 & Sequencing primers for MS2CP-Nhe1 linker-IRES-Puro plasmid with eGFP inserted \\
\hline \multicolumn{2}{|c|}{ CTATATCATGGCCGACAAGCAG } \\
\hline APJ647 & Sequencing primers for MS2CP-Nhe1 linker-IRES-Puro plasmid with TDP-43 inserted \\
\hline
\end{tabular}




\section{Extended Data Table 1: Primer information (continued)}

\begin{tabular}{|c|c|}
\hline Primer & Useage \\
\hline \multicolumn{2}{|c|}{ cagggtgggtttggtaacagtagag } \\
\hline \multicolumn{2}{|c|}{ gtgacagccccaagcctagcagctccc } \\
\hline $\begin{array}{l}\text { APJ654 } \\
\text { gcagccac }\end{array}$ & gcagccacaagcaagggtccagcc \\
\hline \multicolumn{2}{|c|}{ gaacaagcgtcaggtccagcc } \\
\hline \multicolumn{2}{|c|}{ caacactcgagccagacctgcc } \\
\hline \multicolumn{2}{|c|}{ gcaggtgaagccgagagaaacatcc } \\
\hline APJ658 & Sequencing primers for MS2CP-Nhe1 linker-IRES-Puro plasmid with CIZ1 inserted \\
\hline \multicolumn{2}{|c|}{ CAGACCCACCGAACCAAGCTCAG } \\
\hline APJ659 & Sequencing primers for MS2CP-Nhe1 linker-IRES-Puro plasmid with CIZ1 inserted \\
\hline \multicolumn{2}{|c|}{ GGATCTTCCGCTTCTTTCTCCAG } \\
\hline APJ660 & Sequencing primers for MS2CP-Nhe1 linker-IRES-Puro plasmid with $\mathrm{CIZ1}$ inserted \\
\hline \multicolumn{2}{|c|}{ GTTGCACCAGCGTTTGGGCGGAG } \\
\hline tagagcctctgctaaccatgttc & $\begin{array}{l}\text { pBS32 Forward sequencing primer upstream of EcoR1 site } \\
\text { catgttc }\end{array}$ \\
\hline \multicolumn{2}{|c|}{ ctcagtggtatttgtgagccag } \\
\hline \multicolumn{2}{|c|}{ Primers used to assess Xist abundance and splicing } \\
\hline APJ236 & Xist Exon 1 Forward splicing primer (qPCR) \\
\hline \multicolumn{2}{|c|}{ TGCTCCTCCGTTACATCAGA } \\
\hline APJ237 & Xist Exon 2 Reverse splicing primer (qPCR) \\
\hline \multicolumn{2}{|c|}{ GGCAGGAGCACAAAACAGAC } \\
\hline APJ617 & MS2 Forward Primer (qPCR) \\
\hline \multicolumn{2}{|c|}{ CCTAGCCTAGTCTAGATCCGCG } \\
\hline APJ618 & $\begin{array}{l}\text { MS2 Reverse Primer (qPCR) and for PCR amplification of MS2 in vitro transcription template } \\
\text { and for synthesis of Control } 2 \text { RNA in the biotinylated-Isoxazole experiments }\end{array}$ \\
\hline GCAGGC & AAATTAATGTTACTCAG \\
\hline APJ614 & $\begin{array}{l}\text { T7-Forward primer for PCR amplification of MS2 in vitro transcription template } \\
\text { and for synthesis of Control } 2 \text { RNA in the biotinylated-Isoxazole experiments }\end{array}$ \\
\hline
\end{tabular}




\section{Extended Data Table 1: Primer information (continued)}

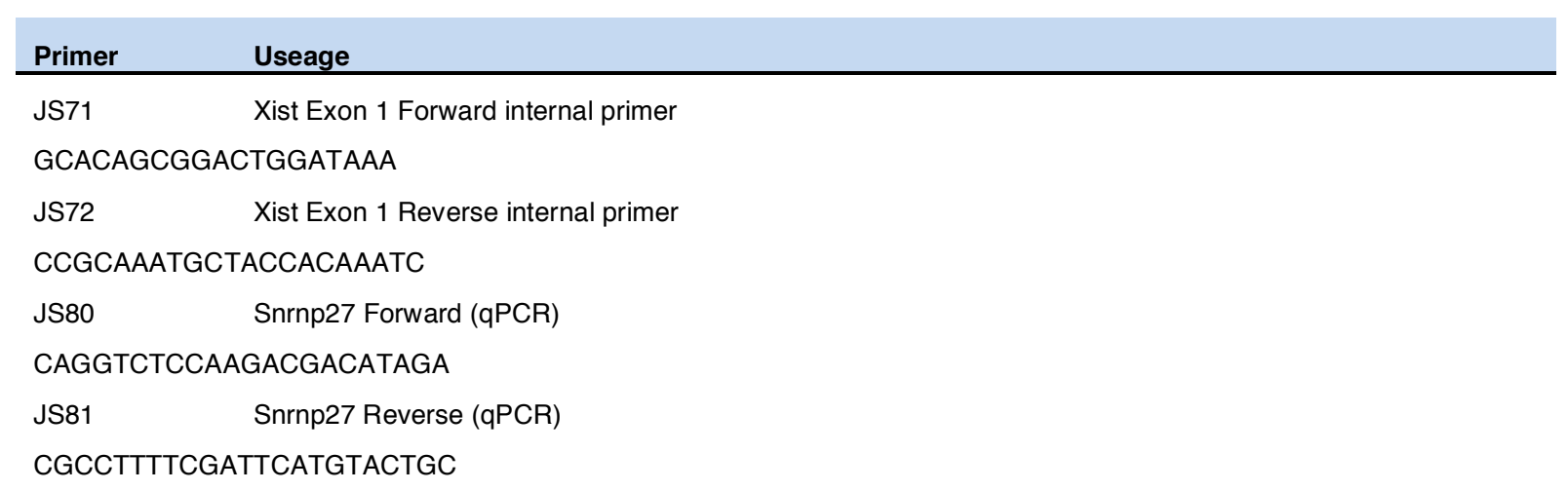

Primers used to amplify in vitro RNA transcription templates

APJ623 Reverse primer for long E repeat in vitro transcription template with Forward primer APJ 614

Short $E$ repeat tempate was amplified using reverse primer APJ624.

CAAGAGAGACACAGACTTGTAAAGAGA

APJ625 T7-Forward primer for short and Long E repeat in vitro transcription templates

TAATACGACTCACTATAGgggtgtgtctatttcttgttttatg

UCLA $41 \quad$ Forward primer for amplification of Control in vitro transcription template

AGCGCGCAATTAACCCTCACTAAAGGtgtgtttcttctgctttggtgagg

UCLA 42 Reverse primer for amplification of Control in vitro transcription template

tcccggctttatagaactgtagac

JS61 Forward T7 primer for PCR amplification of long A repeat in vitro transcription template

TAATACGACTCACTATAGggcttgctccagccatgtt

JS60 Reverse primer for PCR amplification of long A repeat in vitro transcription template

TTCTGAGCAGCCCTTAAAGC

(See also APJ 618 and 614 above)

Primers used to amplify in vitro RNA transcription templates to make RNA FISH probes

UCLA1416 Forward primer for T3 transcription template for Xist RNA FISH probes ttatggctctagattctcctcaag

UCLA1417 Reverse primer for T3 transcription template for Xist RNA FISH probes AGCGCGCAATTAACCCTCACTAAAGGGaagtcagtatggagggggtagagg

UCLA1418 Forward primer for T3 transcription template for Xist RNA FISH probes

tccatggacaagtaaacaaagaac

UCLA1419 Reverse primer for T3 transcription template for Xist RNA FISH probes AGCGCGCAATTAACCCTCACTAAAGGGtatgagggtatgggatcttggtta

UCLA1420 Forward primer for T3 transcription template for Xist RNA FISH probes gatcccataccctcataccctaat 


\section{Extended Data Table 1: Primer information (continued)}

\begin{tabular}{|c|c|}
\hline Primer & Useage \\
\hline UCLA1421 & Reverse primer for T3 transcription template for Xist RNA FISH probes \\
\hline \multicolumn{2}{|c|}{ AGCGCGCAATTAACCCTCACTAAAGGGcttgaaggaccattgaccgtatt } \\
\hline $\begin{array}{l}\text { UCLA1422 } \\
\text { tgctttatggaa }\end{array}$ & $\begin{array}{l}\text { Forward primer for T3 transcription template for Xist RNA FISH probes } \\
\text { atgtgc }\end{array}$ \\
\hline UCLA1423 & Reverse primer for T3 transcription template for Xist RNA FISH probes \\
\hline \multicolumn{2}{|c|}{ AGCGCGCAATTAACCCTCACTAAAGGGggtccgaaaagtaataaggttgtg } \\
\hline \multicolumn{2}{|c|}{ acttttcggaccattgtatctctt } \\
\hline UCLA1425 & Reverse primer for T3 transcription template for Xist RNA FISH probes \\
\hline \multicolumn{2}{|c|}{ AGCGCGCAATTAACCCTCACTAAAGGGgagagcaggtcattcgtcagag } \\
\hline $\begin{array}{l}\text { UCLA1426 } \\
\text { tcccctgctagt }\end{array}$ & $\begin{array}{l}\text { Forward primer for T3 transcription template for Xist RNA FISH probes } \\
\text { atgat }\end{array}$ \\
\hline UCLA1427 & Reverse primer for T3 transcription template for Xist RNA FISH probes \\
\hline \multicolumn{2}{|c|}{ AGCGCGCAATTAACCCTCACTAAAGGGtttcccacagactcatcaccctcag } \\
\hline \multicolumn{2}{|c|}{ ttttaaaaggtgactggatggatt } \\
\hline UCLA1429 & Reverse primer for T3 transcription template for Xist RNA FISH probes \\
\hline \multicolumn{2}{|c|}{ AGCGCGCAATTAACCCTCACTAAAGGGtgatgtaacggaggagcagtag } \\
\hline AM-059 & Forward primer for MS2 probe template \\
\hline \multicolumn{2}{|c|}{ GAGCTTTTCCTATAAGGCATCAGA } \\
\hline AM-060 & Reverse primer for MS2 probe template \\
\hline GGAATAT & AAGACCATGAA \\
\hline
\end{tabular}




\section{Extended Data Table 2: Antibody Information}

\begin{tabular}{|c|c|c|c|c|c|}
\hline Antigen & Company & Cat \# & Assay & Dilution & Additional notes \\
\hline H3K27me3 & Millipore & $07-449$ & Immunofluorescence & $1: 400$ & \\
\hline H3K27me3 & Active Motif & 39536 & Immunofluorescence & $1: 400$ & \\
\hline RNA Polymerase II & Millipore & $05-623$ & Immunofluorescence & $1: 1000$ & \\
\hline Matrin3 & Abcam & ab151714 & $\begin{array}{l}\text { Immunofluorescence } \\
\text { Immunoblot }\end{array}$ & $\begin{array}{l}1: 1000 \\
1: 1000\end{array}$ & Used for CLiP \\
\hline PTBP1 & Abcam & ab5642 & $\begin{array}{l}\text { Immunofluorescence } \\
\text { Immunoblot }\end{array}$ & $\begin{array}{l}1: 1000 \\
1: 1000\end{array}$ & $\begin{array}{c}\text { Used for CLIP/ChIP } \\
\text { and co-IP }\end{array}$ \\
\hline PTBP1 (N-Term) & Douglas Black Lab & $\mathrm{N} / \mathrm{A}$ & $\begin{array}{l}\text { Immunofluorescence } \\
\text { Immunoblot }\end{array}$ & $\begin{array}{l}1: 1000 \\
1: 1000\end{array}$ & \\
\hline PTBP1 (C-Term) & Douglas Black Lab & $\mathrm{N} / \mathrm{A}$ & $\begin{array}{l}\text { Immunofluorescence } \\
\text { Immunoblot }\end{array}$ & $\begin{array}{l}1: 1000 \\
1: 1000\end{array}$ & \\
\hline GAPDH & Fitzgerald & 10R-G109a & Immunoblot & $1: 5000$ & \\
\hline hnRNPU/SAF-A & Abcam & ab20666 & $\begin{array}{l}\text { Immunofluorescence } \\
\text { Immunoblot }\end{array}$ & $\begin{array}{l}1: 1000 \\
1: 1000\end{array}$ & \\
\hline Ezh2 & BD Biosciences & 612667 & Immunofluorescence & $1: 200$ & \\
\hline Flag-M2 & Sigma & F1804 & $\begin{array}{l}\text { Immunofluorescence } \\
\text { Immunoblot }\end{array}$ & $\begin{array}{l}1: 1000 \\
1: 5000\end{array}$ & \\
\hline Flag & Sigma & F7425 & Immunofluorescence & $1: 1000$ & \\
\hline Nanog & Abcam & ab80892 & $\begin{array}{l}\text { Immunofluorescence } \\
\text { Immunoblot }\end{array}$ & $\begin{array}{l}1: 100 \\
1: 500\end{array}$ & \\
\hline CELF1/CUG-BP1 & Abcam & ab129115 & $\begin{array}{l}\text { Immunofluorescence } \\
\text { Immunoblot }\end{array}$ & $\begin{array}{l}1: 1000 \\
1: 5000\end{array}$ & Used for CliP \\
\hline $\mathrm{ClZ1}$ & Novus Biologicals & NB100-74624 & Immunofluorescence & $1: 1000$ & \\
\hline $\mathrm{ClZ1}$ & ThermoFisher & PA527625 & Immunoblot & $1: 500$ & Used for Co-IP \\
\hline TDP-43 & Abcam & ab109535 & $\begin{array}{l}\text { Immunofluorescence } \\
\text { Immunoblot }\end{array}$ & $\begin{array}{l}1: 700 \\
1: 700\end{array}$ & \\
\hline Eed & $\begin{array}{c}\text { Homemade (Arie } \\
\text { Otte) }\end{array}$ & $\mathrm{N} / \mathrm{A}$ & Immunofluorescence & $1: 100$ & Clone M26 \\
\hline Suz12 & Abcam & ab12201 & Immunofluorescence & 1:1000 & \\
\hline Rabbit Normal IgG & Abcam & ab171870 & Immunoprecipitation & 5 ug & \\
\hline
\end{tabular}

